
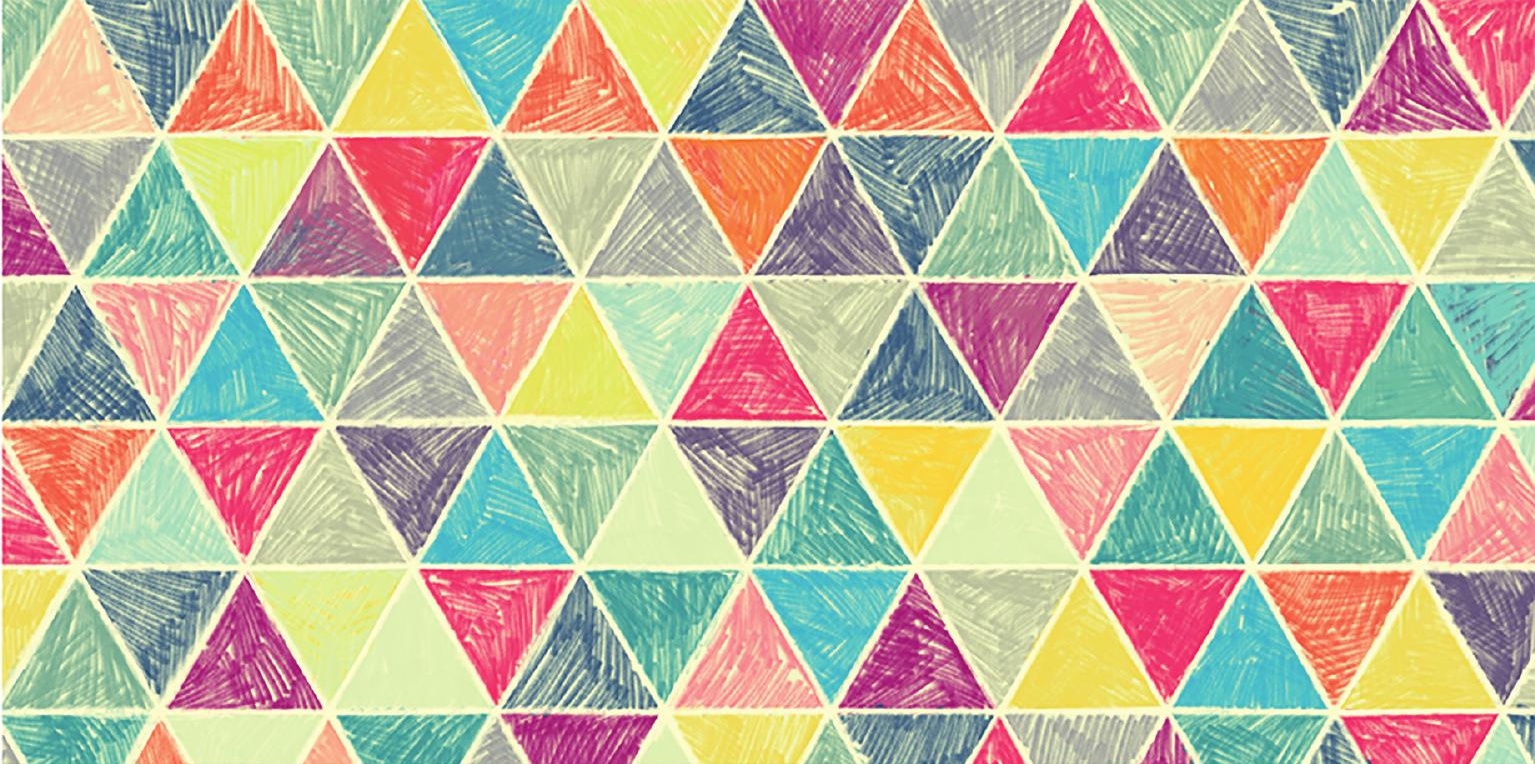

स
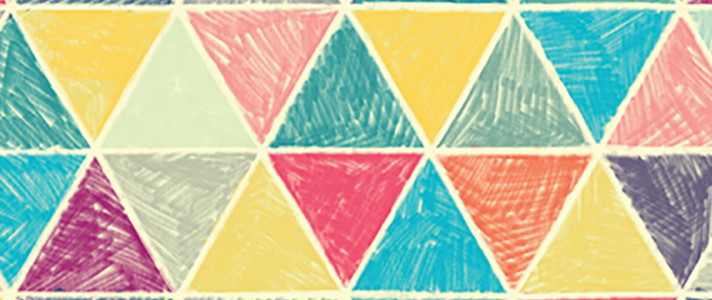

Research in Sexualities

\title{
AGGRESSION IN \\ PORNOGRAPHY
}

\section{MYTHS AND REALITIES}

Eran Shor and Kimberly Seida

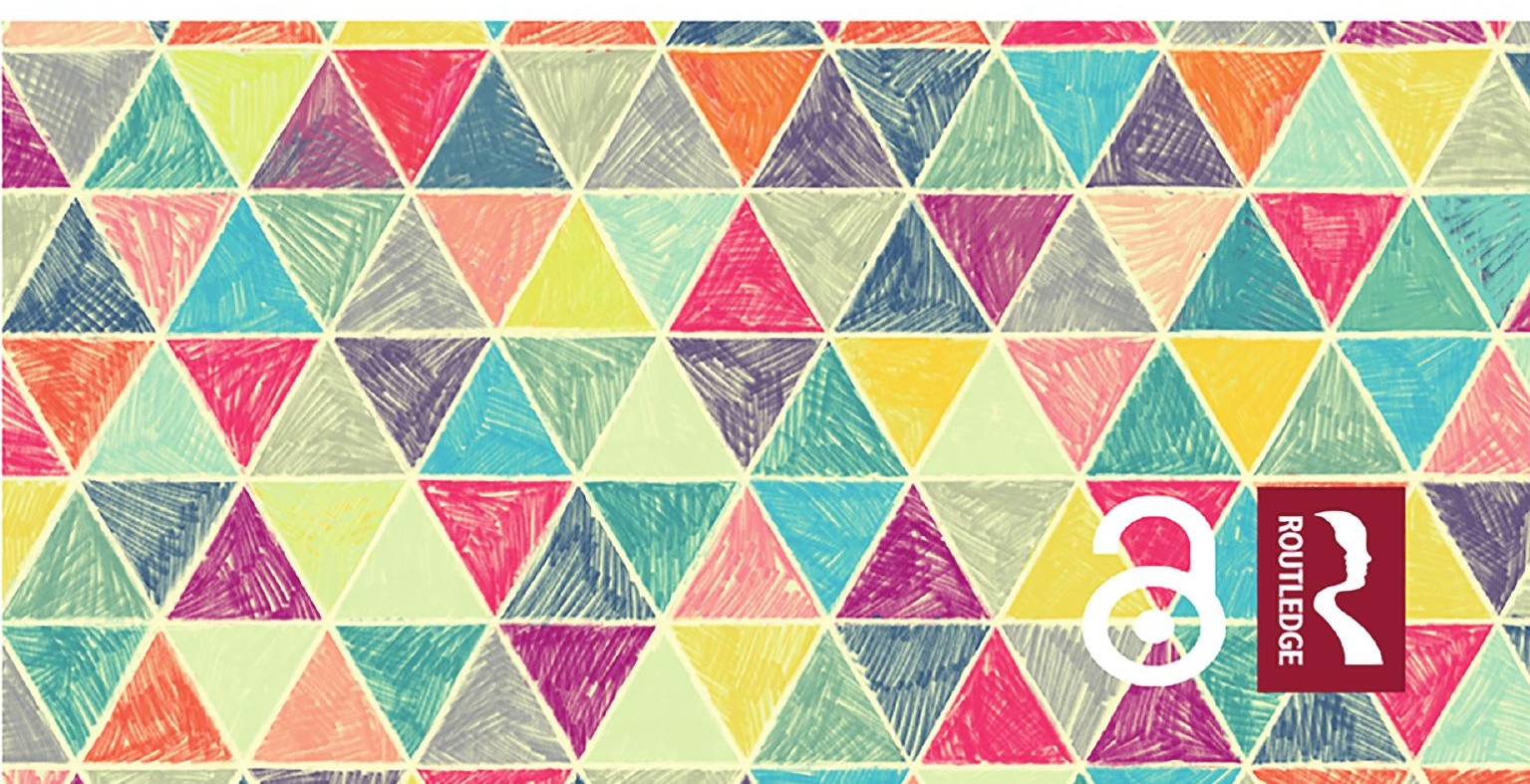




\section{Aggression in Pornography}

Aggression in Pornography focusses on the issue of violence in mainstream pornography and examines what we know, what we think we know, and what are some surprising research findings and insights about the place of violence within pornography today.

The authors first review the modern pornography industry, theoretical claims about pornography as violence, and the ways in which aggression has been defined and measured in previous research. Next, they review the findings of empirical research on violent content in pornographic materials and the potential effects of such content on audiences. The main part of the book relies on systematically collected empirical data, as the authors analyze the content of hundreds of pornographic videos as well as more than a hundred interviews with men and women who regularly watch pornography. These analyses provide surprising insights regarding the prevalence of and trends in violent content within mainstream pornography, the popularity of violent and non-violent content among viewers, and variations in aggression by race and sexual orientation.

As such, Aggression in Pornography will be of interest to students and researchers in sociology, gender and sexuality studies, and media and film studies, as well as to wider audiences who are interested in today's pornography industry and to policymakers looking to devise empirically driven policies regarding this industry and its potential effects.

Eran Shor is a Professor in the Department of Sociology, McGill University, and a William Dawson Chair. In his work, he examines social conflict and violence, focusing on terrorism, ethnic conflicts, and states' repressive policies, as well as on family and sexual violence.

Kimberly Seida is a PhD candidate in Sociology at McGill University. Her dissertation research explores sexual minority women's experiences, focusing on the individual, intersectional, and community-level factors shaping their health and well-being. 


\section{Research in Sexualities}

The Body in French Queer Thought from Wittig to Preciado Queer Permeability

Elliot Evans

Straight Skin, Gay Masks and Pretending to be Gay on Screen Gilad Padva

\section{Aggression in Pornography}

Myths and Realities

Eran Shor and Kimberly Seida

For more information about this series, please visit: https://www.routledge. com/Research-in-Sexualities/book-series/RIS 


\section{Aggression in Pornography \\ Myths and Realities}

Eran Shor and Kimberly Seida 
First published 2021

by Routledge

2 Park Square, Milton Park, Abingdon, Oxon OX14 4RN

and by Routledge

52 Vanderbilt Avenue, New York, NY 10017

Routledge is an imprint of the Taylor \& Francis Group, an informa business

(C) 2021 Eran Shor and Kimberly Seida

The right of Eran Shor and Kimberly Seida to be identified as authors of this work has been asserted by them in accordance with sections 77 and 78 of the Copyright, Designs and Patents Act 1988.

The Open Access version of this book, available at www.

taylorfrancis.com, has been made available under a Creative

Commons Attribution-Non-Commercial-No Derivatives 4.0

license.

Trademark notice: Product or corporate names may be trademarks or registered trademarks, and are used only for identification and explanation without intent to infringe.

British Library Cataloguing-in-Publication Data

A catalogue record for this book is available from the British Library

Library of Congress Cataloging-in-Publication Data

A catalog record has been requested for this book

ISBN: 978-0-367-22640-4 (hbk)

ISBN: 978-0-429-27612-5 (ebk)

Typeset in Bembo

by codeMantra 


\section{Contents}

List of figures vii

List of tables $\quad$ ix

Acknowledgments $\quad$ xi

1 Introduction 1

2 The modern pornography industry:

$\begin{array}{ll}\text { overview and recent trends } & 10\end{array}$

3 Research methodology and definitions 27

4 The effects of pornography on consumers 42

5 Myths about the prevalence of aggression 64

6 Myths about the popularity of aggression $\quad 77$

7 Sexual orientation myths: is aggression less common in same-sex pornography?

8 Race myths: do racial minority women suffer higher rates of aggression?

9 Conclusion: an empirically based account of aggression in pornography

Index 

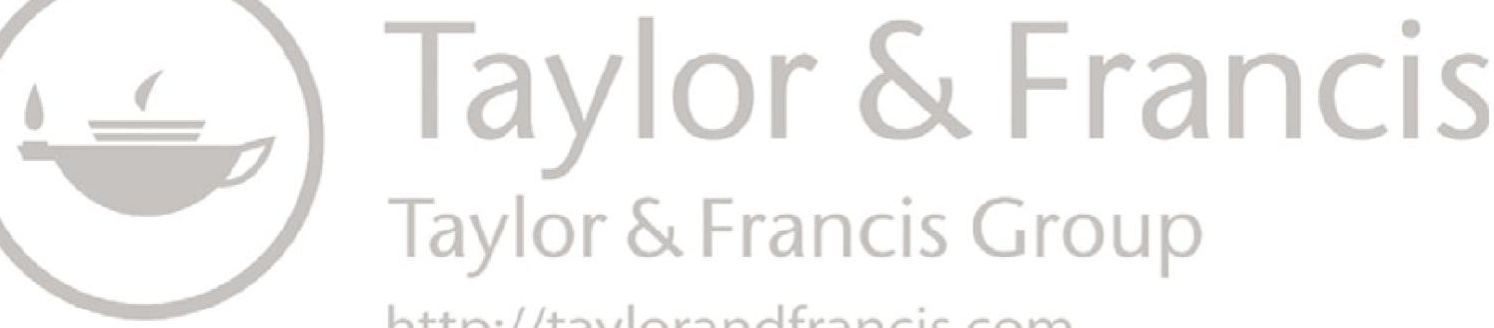

http://taylorandfrancis.com 


\section{Figures}

5.1 Temporal tendencies in aggression $(n=262$ videos $) \quad 72$

5.2 Temporal tendencies in specific aggressive acts ( $n=262$ videos) $\quad 73$

6.1 Aggression and pleasure displays in the most frequently watched $(n=70)$ vs. less frequently watched $(n=76)$ videos

6.2 Aggression and pleasure responses as predictors of viewers' ratings of pornographic videos $\quad 81$

7.1 Aggression in heterosexual and non-heterosexual dyads $(n=210) \quad 95$

7.2 Affection and pleasure displays in heterosexual and non-heterosexual dyads $(n=210) \quad 96$

8.1 Aggression and affection by woman's race 111

8.2 Aggression in video title by racial combination 112

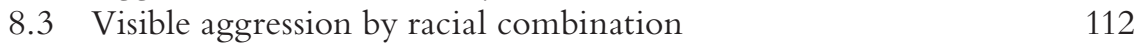

8.4 Non-consensual aggression by racial combination 113

8.5 Physical affection displays by racial combination 114 

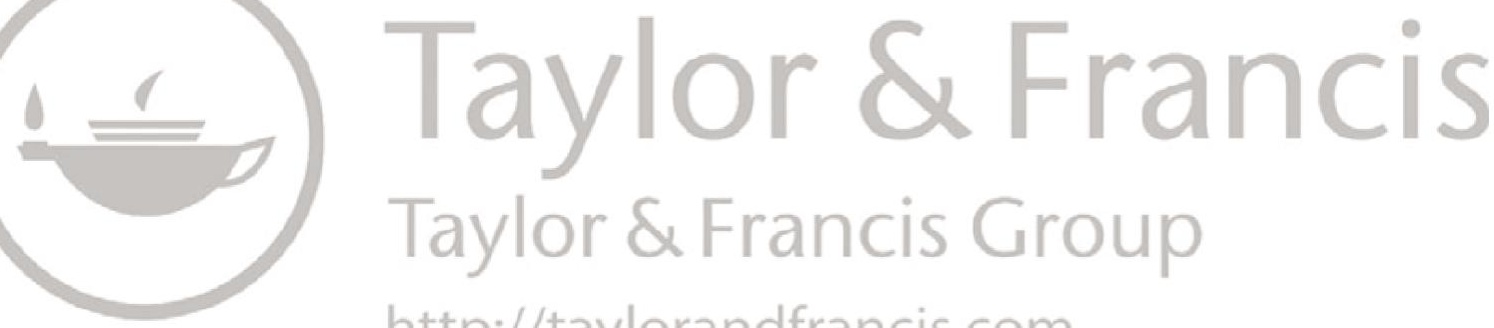

http://taylorandfrancis.com 


\section{Tables}

3.1 Samples details $\quad 29$

3.2 Descriptive statistics of the interviewees' sample $(n=122) \quad 37$

5.1 The prevalence of aggression in PornHub videos 65

5.2 The prevalence of specific aggressive/degrading acts in PornHub videos 

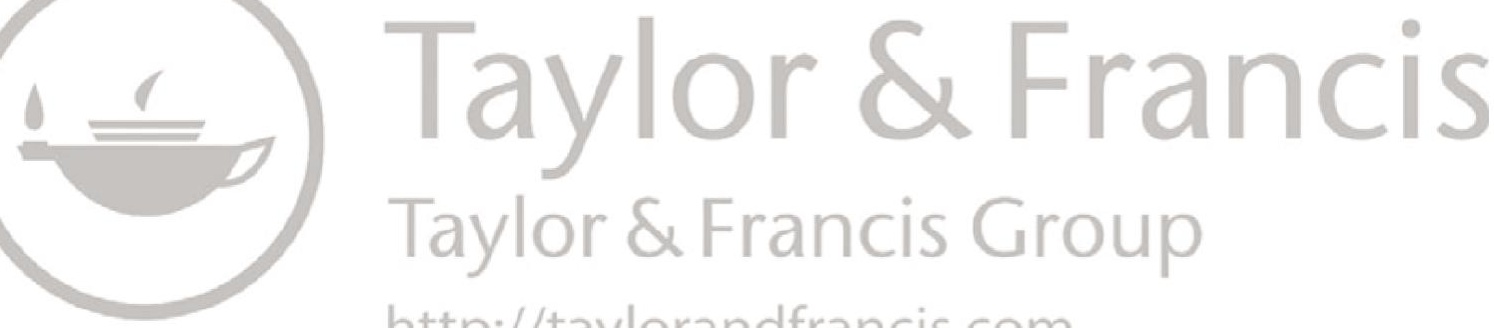

http://taylorandfrancis.com 


\section{Acknowledgments}

The authors would like to thank the three excellent and dedicated research assistants who have worked with us on this book for their invaluable contributions. Golshan Golriz has been most helpful in coding parts of the content of online videos, which serve as the foundation for our quantitative analyses. She has also assisted in the drafting of an earlier version of Chapter 7 of this book. Isabelle Flory, with help from Daniel Sailofsky, has been instrumental in designing, scheduling, and conducting the interviews with pornography viewers, which we cite and rely on throughout the book. 

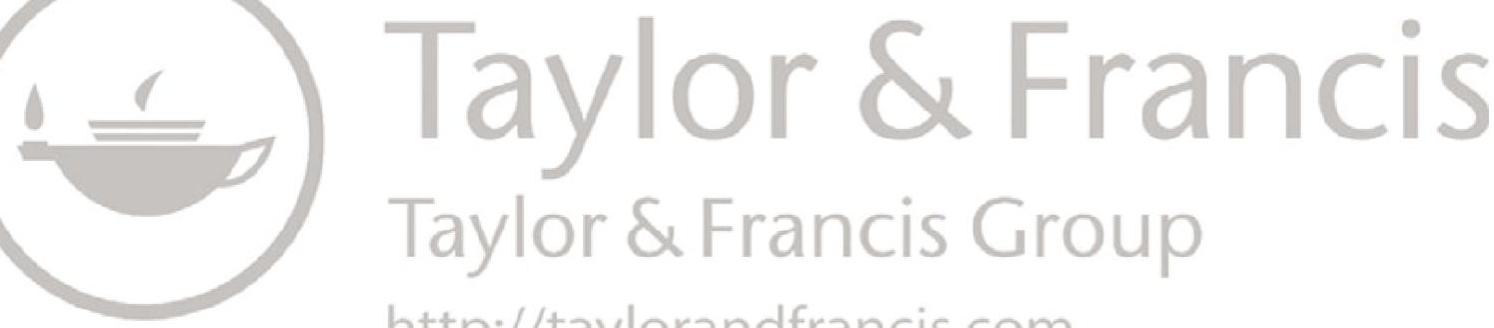

http://taylorandfrancis.com 


\section{Introduction}

Few topics are quite as controversial as sex work and pornography. Over the last few decades, concerns about pornography's psychological, relational, and sociocultural ramifications have entered mainstream debates. The advent of the Internet has increased pornography's accessibility, affordability, and public visibility (Paasonen, 2014) and, in turn, its social and cultural impact. Pornography is now a major subject for discussion in settings ranging from legislative assemblies to daytime talk shows to party conversations and to academic discussions. Regardless of one's personal stance toward pornography, the mainstream pornography industry is a key player in both reflecting and (re)shaping our understandings of gender, sexuality, and intimate relationships.

Pornography is also a particularly fascinating sociological phenomenon, as few topics have entered such a long-standing academic and political stalemate, albeit punctuated by moments of explosive debate (Williams, 2014). Despite its popular significance and controversial nature, pornography-its nature, its use, its effects, and the industry itself-remains relatively under-examined by the scholarly community and in particular by sociologists. This may partly be the result of the stigma often linked with studying sexuality in general and "deviant" sexuality more specifically, with some scholars still being worried that they will become associated with the topic of their study. But it may also be the result of intellectual elitism-the sense that this is not a serious or important enough subject of research. Such elitism might lead to an apprehension by social scientists who wish to study pornography, as they may worry that serious mainstream journals would not be willing to consider their work and social science departments would not offer positions or promotion for those studying such a topic, particularly if they express controversial positions about it.

A cursory overview of both public and academic discussions of and writings on pornography reveals that they have mostly focused on pornography's effects, especially on adolescents and youth. Such a review also highlights concerns about both an assumed increase in violent and degrading content over time and an increased demand among (often male) viewers for aggressive and degrading content. Our main contention in this book is that many 


\section{Introduction}

of these prevailing arguments about the pornography industry, its use, and its effects are primarily ideological and are not supported by systematic empirical research. As such, the field is a breeding ground for decisive ideological statements, which are all too often untested and remain unsubstantiated by rigorous empirical research.

In particular, we identify a number of notable weaknesses in both writings that are critical of the pornography industry and those that support it. Much of the work from both sides remains theoretical or anecdotal, without sound empirical evidence based on research. Some of the studies that do adopt an empirical approach often suffer from loosely defined conceptual definitions, questionable sampling, small sample sizes, and/or selective and biased analyses that are not reflective of the broader industry (e.g. studies that focus on niche or "extreme" materials). All too often, the ideological positions of researchers, journalists, or others writing about the pornography industry, and specifically about aggression therein, greatly influence the theories and facts that they choose to cite, as well as their methodological choices in studying this field.

These shortcomings highlight the need for additional systematic empirical research on aggression in pornography. More specifically, this book examines the validity of claims about temporal trends in aggression, the associations between performers' gender, race, and sexual orientation and aggression in pornography, and the perceptions and preferences of both men and women who regularly watch pornography online. We take another look at some of the most powerful claims and frequently cited narratives about mainstream online pornography and present novel empirical evidence to shed new light on pornography in the 21 st century.

\section{Why did we write this book?}

Our main goal in writing this book is to offer a systematic empirical analysis of some of the main contentions about pornography, and in particular the role of aggression and pleasure in pornography. A deeper empirical understanding of pornography today is important because pornography plays a significant role in many people's desires, fantasies, sexual relationships, and identities. Pornography also provides a fruitful avenue for the exploration of issues such as censorship, freedom of speech, and various ethical issues pertaining to human sexuality. However, we still know surprisingly little about the content of materials produced by this multi-billion dollar global industry, the ways in which these materials are consumed, and how all this affects people's intimate lives, sexual tastes, desires, and identities (Comella, 2013). Indeed, despite pornography's central place in both academic and public discussions, these discussions often fail to engage with empirical evidence and all too often involve moral judgments, relying on selective sampling and the generalization of anecdotes, which are presented as conclusive evidence, while consistently ignoring counterevidence (Weitzer, 2009). In particular, 
we still lack sound and timely empirical research on the prevalence, expressions, and viewer-perceptions of aggression, pleasure, and affection within the pornography industry.

While some scholars and activists who have been writing about pornography since the so-called sex wars of the 1980s have been carefully considering new trends and evidence, infusing their claims with more nuance, others have dug in their heels. Often motivated by firm ideological convictions, these writers and speakers appear determined to either vilify or venerate pornography, leaving little room for nuance and shades of gray. Claims by anti-pornography scholars mostly focus on rates of violence against women, misogyny, and sexual addictions. Conversely, the claims from the opposing camp emphasize women's right to participate in sex work, sexual empowerment and liberation for both men and women, the exploration of various sexual practices and identities, and freedom of expression.

To further complicate matters, some anti-pornography organizations have accused notable pornography scholars, en masse, in holding favorable positions toward pornography (Sociology Lens, 2013) and in absolving the industry of its abusive nature. Such accusations are likely responsible, at least in part, for the political and academic impasse characterizing this research area, as scholars of varying stances are hesitant to conduct and communicate research, fearing that they will be judged by others or identified as anti-feminist supporters of pornography and sexual abuse.

As sociologists, we are trained to look for the broader patterns underlying the conversations we hear in classrooms, coffee shops, and newsrooms. We seek to decipher the messages we receive from peers and media and the ways in which societies shape and reflect life arenas, including identities, relationships, and sexuality. While we fully recognize that our personal and social identities, life experiences, and worldviews impact how we see the world and what we choose to study as researchers, thus precluding "true" objectivity, we nevertheless aim to sift through the "noise" often created in the fray of debates surrounding controversial topics. In order to effectively do so, we need to not only see what the data are showing us but also truly listen to what people are saying and respect their framing of and knowledge about their experiences, rather than merely rely on our own preconceptions.

In the context of this book, adopting such an approach entails thinking about content analyses more inductively, being aware of the potential biases in pre-formed categories and coding schemes, and being willing to consider a wider variety of definitions for controversial terms. It also entailed approaching our qualitative interviews with open minds, seeking to listen to people's opinions and experiences without judgment, and doing our best to create a space in which participants feel comfortable sharing details about topics as private as pornography use, sexual behaviors, fantasies, and desires.

In this book we seek to deepen our understanding and gain greater clarity on this fascinating social and cultural phenomenon. We join a growing tendency among researchers of sex work and pornography to adopt more 
careful, evidence-based approaches to studying pornography, its contents, and its potential effects, while dispelling monolithic, reductive, or inaccurate arguments concerning pornographic material and its consumption (see, for example, Klaassen \& Peter, 2015; Lim, Carrotte, \& Hellard, 2016; McKee, 2015; Rissel et al., 2017; Weitzer, 2005, 2009, 2010, 2011). Our motivation is not only rooted in a sociological inclination to dig deeper when faced with seemingly intractable debates but also in the desire to create dialogue rather than divisiveness and to explore and unpack what some may avoid or quickly dismiss. In sum, we aim to move beyond the "tired binary" (Juffer, 1998), in which pornography is a symbol of either sexual violence or sexual liberation. In doing this, we heed the advice of Linda Williams (2014), when recently articulating the importance of the newly founded journal Porn Studies:

Here we encounter a perennial problem of the field: to write about pornography with any detail or interest is not to automatically advocate its virtues. It would seem that to be interested in pornography... or the intersection of race, class, and gender with their performative identities - is to be cast onto one or the other side of an antiporn-proporn divide that scholarship on pornography, I argue, must get beyond.

(pp. 32-33)

\section{Who are we and what is our stance on pornography?}

Both authors of this book are sociologists with expertise in gender, sexuality, social conflict and violence, and critical media studies. In keeping with the feminist tenet of research reflexivity (England, 1994), we both self-identify as feminists. We deeply believe in the core principles of feminist thought, including women's social, political, and economic equality and the critical importance of addressing all forms of discrimination and maltreatment based on sex, gender, and sexuality. We further recognize the tremendous contributions made by feminists-including those sometimes derisively called "radical feminists"- to women's and men's lives, to the academic enterprise in general, and to the study of sexuality and pornography more specifically. In that vein, we fully acknowledge the complexity of researching and writing about such a contentious issue and its potential to create lines of division within and between feminist communities. We are also familiar (as we discuss in depth in Chapter 4) with the many ways in which pornography use may be associated with both individual and societal deleterious outcomes.

This, then, is not by any means an anti-feminist book. Nor is it, however, an anti-pornography book. We did not set out to write this book in order to vilify the industry or call for its abolition. Instead, we have consciously chosen not to take an explicit stance on pornography. Although we each have our personal views and opinions about the industry, its consumption patterns, and both the short- and long-term effects of pornography use, we refrain from 
deliberately including these opinions in the following pages. In doing so, we attempt to distinguish this research endeavor from much of the work emerging from either side of the "great pornography debate." Consequently, the quantitative and qualitative analyses presented in this book highlight a wide range of perspectives and stories, some of which support previous important work in the field, while others challenge the party line.

We recognize that despite our attempts to remain non-partisan in presenting and interpreting our empirical evidence, we cannot be truly objective. Nor can we control how the arguments and discussions contained in this book will be read by diverse audiences, ranging from staunch critics to ardent defenders of pornography. However, it is our hope that the data and stories presented in this book would help in informing the discussion and in bringing more nuance to what has become a polarized and entrenched debate. Regardless of readers' extant views on pornography and the position with which they approach this research, our goal is that readers will close this book with a more informed perspective and the realization that pornography-like any social, economic, political, and cultural phenomena-needs to be unpacked, rather than ignored, and viewed through a lens that recognizes and values nuance and complexity.

\section{Book overview}

This book is the culmination of more than four years of research. It draws on insights gathered from both quantitative and qualitative content analyses of hundreds of videos from multiple genres of mainstream online pornography found on PornHub, the world's largest pornography streaming website. We complemented this content analysis with data coming from more than one hundred and twenty interviews with regular pornography viewers about their pornography-watching habits, preferences, and opinions. Some of the content analysis results presented in Chapters 5-8 of the book first saw the light of day in three journal articles - two that were published in the Journal of Sex Research (Seida \& Shor, 2020; Shor \& Seida, 2019) and one published in the Archives of Sexual Behavior (Shor \& Golriz, 2019).

The book contains six substantive chapters. Chapter 2 provides an overview of the major historical developments that have shaped the mainstream pornography industry over the last several decades, highlighting the seismic shifts that have occurred within this industry over the last half century. We review some of the major trends in public and academic discourse concerning pornography, noting the gradual mainstreaming and normalization of the industry. We also identify recent trends in the use and content of pornography, including increases in female viewership, the rise of amateur pornography, increasing trends of both younger performers and younger viewers, and the mainstreaming of bondage, discipline, dominance, and submission (BDSM) representations. The chapter gives readers a contextual base and provides background for some of our methodological choices, including the decision 


\section{Introduction}

to focus on freely available mainstream online pornography and our insistence on hearing the voices of both men and women who watch pornography.

Chapter 3 outlines the methodology of the study, providing details on both of its research components - the content analysis of pornographic videos and our interviews with pornography viewers. We explain our sampling choices and provide information about our data and the sample of videos and interviews that we analyzed. We also specify our measurement, coding, recruitment, and interview procedures, while highlighting the ongoing debate within the pornography scholarship around the very definition of aggression in pornography. This definition, in turn, informs the type of conclusions that one might draw from the empirical data. We therefore clarify our choice to adopt two operational definitions, recognizing the potential importance of consent when studying the implications of aggression in sexual scenarios but also the somewhat questionable nature of such free consent within the industry. We also outline the challenges in coding race/ethnicity and in coding aggression in same-sex videos, noting our operational choices in dealing with each of these challenges.

In Chapter 4 we evaluate the extant literature about the "real-life" implications of pornography use, exploring research on both the short- and long-term effects of pornography use. We review both experimental and correlational studies that attempted to assess the effects of pornography exposure on sexual aggression and on approaches toward gender equality. We also examine qualifying and moderating factors in this relationship as well as the evidence regarding the effects of pornography use on adolescents and youth. Finally, we add our own original contribution to this body of literature by drawing on our qualitative interviews. These interviews demonstrate that viewers' delineation of pornography as fantasy and their judgments about the nature of consent clearly influence their assessments regarding the impact of viewing on their personal and sex lives.

In Chapter 5 we present some of our most revealing and important findings focusing on the analysis of data regarding the prevalence of aggression in pornography. The chapter investigates and re-examines a few notable commonly held beliefs and misconceptions about the content of today's online pornography. More specifically, we re-examine the common claim that most mainstream pornographic videos contain aggression as well as the claim that the frequency and severity of aggressive acts has been increasing over time. Our data and analyses show that both of these claims are unsubstantiated. Regardless of one's definition of aggression, the majority of the most popular videos do not contain aggression and, again depending on definition, rates of aggression have either been stable or show a downward trend over the last decade.

In Chapter 6 we rely on both an analysis of viewers' online ratings and an analysis of our own interviews with regular viewers of pornography to re-examine claims about viewer (particularly male viewers) preferences for aggressive content and to reassess the importance of affection and pleasure displays in popular videos. We report a number of surprising and counterintuitive 
findings, leading us to question some of the very fundamental assumptions that many hold about the pornography industry. More specifically, we show that most viewers prefer to watch videos that contain less aggression, as demonstrated by the number of views for such videos, the ratings they receive from viewers, and viewers' own pronounced preferences about videos containing aggression. We also show that it is women, not men, who are relatively more likely to express interest in various forms of aggression.

Next, Chapter 7 conducts the first-of-its-kind systematic comparison of aggression between mainstream pornographic videos featuring male/female sexual partners and those featuring male/male and female/female partners. We assess the prevalence and intensity of aggression in each of these three dyadic couplings and explore how pornography viewers think about aggression in each of them. Through these comparisons, we also engage with the broader assumptions that underpin much of the critical scholarship on pornography, particularly the contention that pornography is about men's violence against women. These analyses also highlight the relevance and limits of dominant sexual and gender scripts when analyzing across subgenres of mainstream online pornography, especially as these scripts pertain to non-dominant partner pleasure displays and to affection between partners.

In the last substantive chapter of the book, Chapter 8, we address the contention that aggression in pornography mirrors the racial and gendered hierarchies found within our broader culture. We explore common notions about higher rates of on-screen aggression toward minority performers when compared to white performers in pornography and present data that put some of these notions in question. We also look at the role of male performers and examine the likelihood of different men of different ethnicities to be cast as the aggressors. In addition to looking at men and women separately, we also pay special attention to the interaction of gender and race. Thus, we examine how same-race and interracial couplings influence the portrayals of aggression but also displays of affection and pleasure between sexual partners. Finally, as in the other empirical chapters, we also ask how pornography viewers perceive the relationship between gender, race, and aggression.

We end the book by reiterating our call for a more nuanced and empirically informed debate about aggression in pornography and about the pornography industry more generally. We argue that the study of pornography and the academic and public debate about the issue must not ignore or underestimate the "darker" and harmful aspects of this industry. Nor should it, however, be dedicated to the denigration of all pornography, ignoring both the ways it has changed and diversified over time and viewers' own complex and multifaceted accounts of their viewing experiences and perceptions.

Before beginning our exploration, we would like to add a word of caution about the conclusions and insights that one might draw from this study. The book addresses a wide array of trends, assumptions, beliefs, and concerns regarding both aggression and pleasure in freely available mainstream online pornography. However, it is important to emphasize that our analyses are 


\section{Introduction}

based on a particular sample of videos (non-paid online videos) and interviewees (regular viewers of pornography online who volunteered to participate in the study). It is also rooted in a specific moment in time (the digital era) and a relatively limited geographic context (largely North America, though many of our interviewees do hail from other nations worldwide). Consequently, one should exercise caution when trying to generalize the findings and claims of this book to content and subgenres that are not freely available online, to larger groups of individuals (both those who watch pornography and those who do not), to different time periods, and to other sociocultural and geographic contexts.

\section{References}

Comella, L. (2013, June 14, 2017). Why pornography deserves its own academic journal. Retrieved from https://psmag.com/social-justice/why-pornographydeserves-its-own-academic-journal-57816

England, K. (1994). Getting personal: Reflexivity, positionality, and feminist research. The Professional Geographer, 46(1), 80-89.

Juffer, J. (1998). At home with pornography: Women, sex, and everyday life. New York, NY: New York University Press.

Klaassen, M., \& Peter, J. (2015). Gender (in)equality in internet pornography: A content analysis of popular pornographic internet videos. Journal of Sex Research, 52(7), 721-735.

Lim, M., Carrotte, E., \& Hellard, M. (2016). The impact of pornography on gender-based violence, sexual health and well-being: What do we know? Journal of Epidemiology and Community Health, 70(1), 2-4.

McKee, A. (2015). Methodological issues in defining aggression for content analyses of sexually explicit material. Archives of Sexual Behavior, 44, 81-87.

Paasonen, S. (2014). Diagnoses of transformation: "Pornification," digital media, and the diversification of the pornographic. In L. Coleman \& J. Held (Eds.), The philosophy of pornography: Contemporary perspectives (pp. 3-16). Lanham, MD: Rowman \& Littlefield.

Rissel, C., Richters, J., de Visser, R., McKee, A., Yeung, A., \& Caruana, T. (2017). A profile of pornography users in Australia: Findings from the second Australian study of health and relationships. Journal of Sex Research, 54(2), 227-240.

Seida, K., \& Shor, E. (2020). Aggression and pleasure in opposite-sex and same-sex mainstream online pornography: A comparative content analysis of dyadic scenes. Journal of Sex Research. doi:10.1080/00224499.2019.1696275.

Shor, E., \& Golriz, G. (2019). Gender, race, and aggression in mainstream pornography. Archives of Sexual Behavior, 48, 739-751.

Shor, E., \& Seida, K. (2019). 'Harder and harder'? Is mainstream pornography becoming increasingly violent and do viewers prefer violent content? Journal of Sex Research, 56, 16-28.

Sociology Lens. (2013, February 26, 2017). Revisiting the porn wars. Retrieved from https://www.sociologylens.net/topics/communication-and-media/revisitingthe-porn-wars $/ 11832$

Weitzer, R. (2005). Flawed theory and method in studies of prostitution. Violence Against Women, 11(7), 934-949. 
Weitzer, R. (2009). Sociology of sex work. Annual Review of Sociology, 35(1), 213-234.

Weitzer, R. (2010). Sex for sale: Prostitution, pornography, and the sex industry. New York, NY: Routledge.

Weitzer, R. (2011). Sex trafficking and the sex industry: The need for evidence-based theory and legislation. The Journal of Criminal Law \& Criminology, 101(4), 1336-1370. Williams, L. (2014). Pornography, porno, porn: Thoughts on a weedy field. Porn Studies, 1(1-2), 24-40. 


\section{The modern pornography industry

\author{
Overview and recent trends
}

Pornography is a protean term but may be defined as "Material deemed sexual, given the context, that has the primary intention of sexually arousing the consumer" (Ashton, McDonald, \& Kirkman, 2019, p. 14). Defined in such broad terms, documented pornographic artifacts date back to ca. 5200 BCE (Head, 2019), although others trace its origins only to the 19th century (Kendrick, 1997). However, the modern pornography industry did not take form until the 1960s and has since undergone massive shifts. In this chapter, we review the historical developments in pornographic media, popularity, and consumption patterns since the late 1960s. In doing so, we describe the central role of technological developments such as the advent of the Internet and freely available and accessible tube and streaming sites in (re)shaping both the industry and its place in society over the last 50 years.

\section{Technological developments and historical shifts in the pornographic industry over the last 50 years}

The history of the modern pornography industry can be broadly divided into three "eras" (Tarrant, 2016). The late 1960s until the early 1980s marked the "golden age" of pornography. During this era, sexually explicit films, including Andy Warhol's Blue Movie (1969) and Gerard Damiano's Deep Throat (1972), entered the mainstream film industry and garnered relatively positive attention. The commercial success of Deep Throat was central to the evolution of the pornography industry in the United States and catalyzed the increased production of hard-core films throughout the 1970s (Barnett, 2018). This mainstream attention enabled pornographers to increase production budgets, distribution, and profits. High production values and complex storylines not only increased the mainstream popularity of films but also lent them a degree of artistic legitimacy in the eyes of critics and the public. Consequently, the pornography produced during the "golden age" is often referred to as "porno chic" (DeLamater \& Plante, 2015).

The invention and increased availability of videocassette recorders (VCRs) in the early 1980s, ended pornography's "golden age" (Lehman, 2003) and launched the "video era" of porn, which lasted until the early 1990s. 
Videocassettes allowed for private, in-home viewing, while the camcorder enabled virtually anybody to create their own pornography. These technologies led to widespread dips in attendance of pornographic films in public theaters and ever-increasing at-home viewing. The availability of cable networks and specialty channels in the 1990s further increased accessibility and privacy, allowing consumers to retrieve content directly from home (Coopersmith, 1998; Morichetta, Trevisan, \& Vassio, 2019).

The adult film industry used the Video Home System (VHS) to increase public exposure, relying on the technology's "length" capacity (two to four hours) and low production costs. Indeed, the adult film industry was a key catalyst in the widespread purchase of VHS-compatible VCRs. Only after the adult film industry had flooded the marketplace with scores of adult movies on VHS, and enough people had purchased VCRs in order to watch the videos, did the mainstream film industry consider home movie viewing as a secondary market for their products (Konow, 2007). As more and more people bought VCRs and VHS tapes, prices for each became increasingly affordable, and the adult industry flourished. In the documentary film The Other Hollywood, former porn star Tim Connelly recalled that with the industry's transition from film to video, "the production costs became a tenth of making a film, and they pushed out the same number of copies, raking in huge profits" (McNeil, Osborne, \& Pavia, 2005).

After relying primarily on VHS technology in the 1980s, in the mid-1990s the industry began to utilize DVDs as a new distribution format. As with the VCR and VHS technologies, the adult film industry was a key component in the broader commercial success of DVDs. With this new technology, the production values and overall quality of the films increased while keeping production costs relatively low (Konow, 2007). However, within years, this new technology would become outdated, gradually giving place to the new age of online pornography consumption.

The invention of the digital camera and the rapid spread of the Internet, starting in the early 1990s, ushered in the third and current digital era. Within a short period of time, the Internet has become increasingly available, affordable, and capable of storing massive volumes of material. These swift technological developments have made pornography more accessible, affordable, and anonymous (Cooper, 1998) and have been central to creating what some have estimated as a $\$ 100$ billion global industry (Wosick, 2015). Internet pornography has flourished over the past decade and has become the main source of pornography consumption (Hald, Malamuth, \& Lange, 2013). According to some estimates, it now accounts for about $30 \%$ of all Web traffic (HuffPost, 2017), and according to others, one quarter of all Internet users view pornography online in any given month (Paul, 2010). Recent statistics from PornHub, one of the world's most popular "tube sites" for freely available pornography, reflect the ubiquity of pornography use: in 2019, about 39 billion searches were performed, the site was visited 42 billion times (115 million average daily visitors), and 6.83 million new videos were 
uploaded (PornHub, 2019). When comparing international studies, the estimated percentage of men who view pornography ranges from $50 \%$ to $99 \%$ (Hald, Seaman, \& Linz, 2014).

The move away from pay-per-view and rented pornography to freely available material, usually on "tube" sites, also known as the move to Web $2.0^{1}$ (Paasonen, 2010), has been one of the key shifts in the pornography industry over the last decade (Wilkinson, 2017). The Web 2.0 of pornography is largely a duopoly, with most sites owned by either MindGeek (the parent company of popular websites such as PornHub, RedTube, YouPorn, and dozens of others) or WGCZ Holding (the parent company of websites like XVideos and XNXX) (Morichetta, Trevisan, \& Vassio, 2019; Tarrant, 2016). Fueled by technological developments such as streaming, torrenting, and the increasing use of handheld technologies as viewing platforms, more and more people are viewing pornography and fewer and fewer are paying for it. Some studies estimated that already a decade ago, $80 \%-90 \%$ of Internet pornography viewers were accessing only free material, such as bootleg copies, free samples of paid material, or amateur videos (Cowan, 2010; Doran, 2008). Given the continuous expansion of free materials and platforms, their relative share and popularity have likely further increased since. In addition, the advent and increasing ubiquity of handheld electronics with Internet connection (primarily smartphones) offer users unprecedented access to free pornography at all times and with few limitations. PornHub's (2018) statistics show that in 2019 smartphone and tablet access accounted for more than $80 \%$ of PornHub's worldwide visits (PornHub, 2019).

\section{Mainstreaming and normalization}

These technological developments, particularly the use of mobile devices in order to access pornography, have played a role in the sociocultural mainstreaming and normalization of pornography. Mainstreaming is one of the most commonly discussed trends in public and academic debates about pornography. It is sometimes referred to as cultural "pornification" (Paul, 2005). This term refers to "the increased visibility of hard-core and soft-core pornographies and the blurring of boundaries between pornographic and mainstream" visual norms (Paasonen, Nikunen, \& Saarenmaa, 2007, p. 8). Paul (2005) herself has argued that our "entire culture has become pornified [...] the aesthetics, values, and standards of pornography have seeped into mainstream popular culture" (p. 1).

One example of cultural pornification is the increasing fame and popularity of mainstream and pornographic female performers (see McNair, 2002). Kim Kardashian, a globally known media personality who gained fame partly due to a sex tape, is perhaps the most notable example (Church Gibson, 2014). Other examples include Jenna Jameson, a former pornography performer, whose memoir landed her on daytime talk shows, and Sasha Grey, another performer who has made appearances in various television series. 
Other commonly cited examples of cultural pornification include sexualized print and media advertising (Long, 2016), music videos (Paasonen, Nikunen, \& Saarenmaa, 2007), the overlap between pornography and online gaming (Martinez \& Manolovitz, 2010), and "pornostyle" fashion, where the goal is to be 'sexy' rather than 'stylish' (Church Gibson, 2014). In each of these examples, sexuality and product are blended in an effort to maximize profits (Walker, Makin, \& Morczek, 2016).

This steadily increasing process of pornification has also gradually led to the normalization of pornography. For some, this normalization holds promise, particularly for women's sexual expression (Weinberg, Williams, Kleiner, \& Irizarry, 2010). For others, it normalizes sex acts women may find painful or degrading (Harrison \& Ollis, 2015) and naturalizes "real-world" violence against women (Long, 2016). Jensen (2007), an ardent critic of pornography, has argued that normalization renders the industry uncontroversial, ignores its negative impacts, and discredits opponents as fanatical or motivated by religion and/or traditionalism. He suggested that in a patriarchal culture, the normalization of pornography leads to the subsequent normalization of crueler and degrading sexual acts without much viewer backlash. For Jensen, pornography already represents mainstream North American values, which include corporate capitalism, the patriarchal logic of domination and subordination, and white supremacy. Along with concerns about normalization, some scholars worry that the mainstreaming of pornographic scenes, scripts, and stars will increasingly blur the lines between sexual fantasy and reality (Boyle, 2011; Snape, 2010). Such processes, in turn, may strengthen pornography's capacity to both reflect and (re)shape sexual desires, attitudes, and behaviors. We discuss these potential effects of pornography in Chapter 4.

\section{Recent trends in pornography use and content}

The tendency to see the pornography industry as a monolith (Mercer, 2004), along with the social stigmas attached to both researching and discussing it openly, make it relatively difficult to ascertain distinct trends in pornography consumption. As Walker, Makin, \& Morczek. (2016, p. 658) contend:

We know little regarding actual trends in the consumption of pornography in the United States... this is compounded by the unfortunate inability to disaggregate pornography into a heterogeneous construct. Therefore, we base several sociological assumptions on the impact of pornography (e.g., hypersexualization, objectification, and effects on the psychology and behavior of men), without understanding variations in interest that surrounds and drives the pornographic market and its associated genres.

We agree with this broad characterization by Walker et al. regarding our knowledge about pornography consumption in North America and elsewhere, 
which largely motivated this book and our own research on pornography. Still, the existing literature offers notable observations on four broad developments in the demographics of pornography's viewership, viewers' preferences, pornographic performers, and the content available to viewers. These include an increase in female viewership, increases in the supply and consumption of amateur pornography, younger performers and consumers, and the mainstreaming of BDSM. We expand on each of these trends below.

\section{Increases in women's viewership}

In tandem with their broader role in normalizing pornography, digital technologies may be enabling women's consumption of online pornography and normalizing women's sexual desires (McKeown, Parry, \& Light, 2018). Recent studies suggest that pornography use among women has been on the rise (Fradd, 2017; McKee, Albury, \& Lumby, 2008; McKeown, Parry, \& Light, 2018). However, this trend has not often been discussed or researched (Attwood, 2005). Some studies have suggested that $31 \%-41 \%$ of adult women in the United States and Canada use pornography, ranging from occasional to frequent use (Albright, 2008; de Cadenet, 2015). Others have suggested that rates may be even higher cross-culturally, with a sizable proportion of women (between 30\% and 86\%) using pornography (Hald, Seaman, \& Linz, 2014).

Other recent work suggests that women represent one of the fastest-growing demographic groups of online pornography consumers (Chowkhani, 2016; Penley, Shimizu, Miller-Young, \& Taormino, 2013; Smits, 2016). PornHub, one of the world's largest pornographic tube sites, revealed in its most recent annual analytics review, that that $32 \%$ of the entries to the website in 2019 were by women, up from 29\% in 2018 and 26\% in 2017 (PornHub, 2019).

In line with gendered expectations, female viewers of PornHub were twice as likely as male viewers to watch "Romantic" themed videos. More surprisingly, however, the PornHub reports also noted that "women were twice as likely [as men] to watch content featuring 'Gangbang' and 'Double Penetration"' (PornHub, 2018). Further challenging the notion that women are looking only for mild pornography, in 2014, both "rough sex" and "bondage" made the list of the top-16 searches by women, while neither of these search terms was in men's top-16 searches (PornHub, 2015).

de Cadenet (2015) recently conducted one of the largest online surveys of female pornography viewers, involving over 3,000 women. She found that most women used pornography for their own pleasure or to discover aspects of their sexuality and most of them viewed it by themselves, not with others. These findings stand in contrast to assumptions that women only watch pornography to please their partner or avoid it altogether (see Campbell \& Kohut, 2017). It is important to note, however, that most female viewers of pornography expressed concerns about how the industry treats women and many of them believed that it perpetuates negative stereotypes. At the same time, many respondents in de Cadenet's study said that pornography 
helped them in learning about different types of sex and sexuality. Similarly, McKeown and colleagues (2018) found that women connected societal normalization of pornography to several positive effects, including discovery and validation of their personal sexual desires. Some scholars are critical of such accounts, rejecting the idea that users "discover" inherent aspects of their sexuality through pornography. Instead, they argue that pornography use actively contributes to the construction of such desires (see Foucault, 1992). Still, the findings presented above may serve to mitigate some of the concerns expressed by anti-pornography scholars about the deleterious impacts of normalization, particularly concerning sexual objectification and aggression (see Jensen, 2011; Long, 2016).

\section{Increases in the supply and consumption of amateur pornography}

Over the last decade, amateur pornography has become a major genre in Internet pornography. It threatens the dominance and profits of commercial pornography (Ruberg, 2016; Stella, 2016) and has a considerable degree of appeal for users (Paasonen, 2010; van Doorn, 2010). In contrast to the high-budget "porno chic" of the 1970s and 1980s (Bronstein \& Strub, 2016; DeLamater \& Plante, 2015), and the "Vivid girls" featured in much of the mainstream pornography of the 1990s (Tarrant, 2016), the current landscape of online pornography features a significant amount of amateur content (Effron, 2011; Wilkinson, 2017). It is difficult to determine the prevalence of amateur porn, in part because it is unregulated and in part because professional studios often attempt to mirror the amateur aesthetic (Forrester, 2016). New media platforms, such as tube sites, have enabled users to upload their own amateur content, blurring the boundary between producer and consumer (Paasonen, 2010; Wilkinson, 2017). Amateur pornographers, who upload their own content, may be motivated by sexual expression, the thrill of public performance, or viewer admiration (Ruberg, 2016).

For some, the growth of amateur content signals a "democratization" of online pornography, entailing increasingly horizontal relationships and dynamics between consumers and producers (Ritzer \& Jurgenson, 2010; Wilkinson, 2017) and a potential to diversify aesthetics (e.g. body types and appearances) (Jacobs, 2007; Miller-Young, 2007). The increase of usergenerated materials may therefore be diversifying mainstream pornography beyond the "traditional" genres overwhelmingly produced by and for heterosexual men (Wilkinson, 2017; see also Attwood, 2007; McNair, 2013; Taormino, Parrenas, Shimizu, \& Miller-Young, 2013). Others, however, are less enthusiastic about this trend. In her critique of the "democratization" thesis, Ruberg (2016) argues that valorizing unpaid amateur pornography can promote stigma concerning the legitimacy of paid sex work and can devalue the sexual work and expression of those who cannot afford to participate in unpaid sex work. According to Ruberg, sex work is labor. Therefore, focusing on the "democratizing" potential of amateur pornography, rather than on 
issues of compensation, invisibilizes the "mechanisms of capitalism that function beneath the Internet's abundant wealth of seemingly free erotic content" (p. 158).

Alongside the rise of user-generated amateur pornography, the genre of professional amateur, or "pro-am" pornography, has also proliferated. "Pro-am" is commercial pornography that is marketed as "amateur," yet features paid actors (Bauer, Gradus, \& Jones, 2015; Wilkinson, 2017). In this subgenre, professional actors imitate amateur conventions and styles (Penley, 2004). According to the 2015 documentary Hot Girls Wanted (Bauer et al., 2015), thousands of 18 - to 20 -year-olds enter the US pro-am industry each year, with one talent agent noting that "every day a girl turns 18 , and every day a new girl wants to do porn. I will never run out." "Pro-amateur" pornography is one subtype of the broader genre of "gonzo" pornography. With few notable exceptions (see Lodder, 2016), this genre is characterized by poor recording quality; little to no editing; realistic conventions and images; often shooting from a male subjective viewpoint; use of digital technologies (e.g. GoPro, mobile phones); little to no storyline; and first-person narration, often by the male individual filming the scene (Stella, 2016). Gonzo can take several forms, including industrial (i.e. featuring professional actors), amateur, or a hybrid of the two (e.g. pro-am). Some scholars have argued that gonzo is synonymous with violent and abusive pornography (see Dines, 2006, 2012). Indeed, it appears that at least some amateur gonzo consists of men secretly filming their sexual partners or sex workers without their consent (Ruberg, 2016; Shimizu, 2010). Furthermore, much of gonzo pornography-both industrial and pro-am-hinges on men's sexual interests. Stella (2016) argues that there is virtually no gonzo in which women are directing the filming and/or controlling their own representation (e.g. by holding the camera), although our own content analyses of online videos suggest that such cases do exist, perhaps in growing numbers.

Some scholars have suggested that the proliferation of "professional amateur" (pro-am) pornography is the industry's response to the popularity of "real" amateur pornography and is a consequence of keeping pace with consumers' demand for more "authentic" scenes (Barcan, 2002; Wilkinson, 2017). In contrast to gonzo and pro-am genres, in "truly" amateur pornography, couples often construct the scene more or less jointly, which may work toward rebalancing power and autonomy (Stella, 2016). Others, such as Dines (2010), see amateur pornography as part and parcel of the mainstream pornography industry, given their overlapping distribution channels (e.g. tube sites) and the blurry lines between amateur and "pro-am" (see also Paasonen, 2010).

\section{Younger performers and consumers}

A third notable trend in the pornography industry is the growing involvement of younger people with pornography, on both the production and consumption side. These tendencies are expressed in the growing popularity of 
pornography featuring very young or young-looking women and the increasing rates of adolescents and even children who consume pornography.

Pornography has long featured or portrayed very young women. Notable examples are Playboy's college-girls series in the 1970s and Larry Flynt's Barely Legal magazine, which launched in 1993. However, some evidence suggests an increase over time in the relative interest in pornography featuring youth or actors who appear to be young. Levy and Dines (2013) performed a Google trend analysis showing that searches for "teen porn" more than tripled between 2005 and 2013, growing to one-third of all daily porn searches in 2013. Similarly, Walker, Makin, and Morczek (2016) found that interest in youth-oriented pornography increased considerably between 2004 and 2014. These increases have turned videos featuring very young performers into arguably the most popular genre of pornography. Ogas and Gaddam (2011) and Grey (2015) have noted that the most popular category of sexual searches online-by a large marginwas "youth" or "teen." Globally, "teen" was ranked as the second most searched term (Pegg, 2016) and has been the top PornHub category (i.e. the category receiving the highest amount of traffic) since 2013 (PornHub, 2018).

Critics of pornography have referred to such trends as a cultural "pedophilic drift" (Gilkerson, 2013; Grey, 2015) and "childification" of women (Dines, 2009). These scholars contend that the usage of terms (in titles, tags, or scripts) such as "barely legal," "jailbait," or "sweeties" accentuates youth and childlikeness and normalizes sexual desire for underage girls (Fradd, 2017). Beyond the content itself, some have focused on the societal influence of mainstream pornography's visual norms, alluding to the rise in "designer vagina" surgeries as evidence (Sanghani, 2014; Walker, Makin, \& Morczek, 2016). For instance, there are indications of increasing rates in Western countries of medical procedures that arguably make women's anatomy appear younger, such as labiaplasty, which reduces the size of women's labia minora (Miklos \& Moore, 2008; Sanghani, 2014); vaginoplasty, which tightens the vagina (Braun, 2009); and hymenoplasty, a surgical restoration of the hymen (Braun, 2009). These procedures are often attributed to the influence of pornography and its frequent infantile representations of the female body (Sanghani, 2014).

Taken together, there is some evidence to suggest that interest in youthoriented pornography has increased over the past decade (Walker, Makin, \& Morczek, 2016) and that this increase appears to coincide with the aforementioned processes of cultural pornification (see Gill, 2008, 2012). That said, the sexualization of underage girls (or women who are made to appear younger than 18 using indicators such as pigtails, school uniforms, or braces) is not unique to the pornography industry. It is also widespread in advertising, fashion, music videos, and popular culture, which all predate the Internet (Schwyzer, 2012).

In addition to concerns about performers' young age (or attempts to make performers look very young), the proliferation and increasing accessibility of online pornography have catalyzed social concerns that children and youth 
are being exposed to and are accessing pornography at increasingly younger ages. Studies in the United States (Harrison \& Ollis, 2015) and in Canada (McIntyre \& Clark, 2015) suggest that the average age of initial exposure [often accidental or involuntary (Lewis, Somers, Guy, Watchirs-Smith, \& Skinner, 2018)] to online pornography is 11. "Digital natives" (Wolak, Mitchell, \& Finkelhor, 2007) is a term used for youth and young adults who have been born into a world connected by the Internet and smartphones, which facilitate their access to and use of online pornography. Internet-enabled devices have allowed people of all ages to increasingly consume, create, and distribute sexually explicit materials (Flood, 2007; Lo \& Wei, 2005; Wolak, Mitchell, \& Finkelhor, 2007). Recent studies illustrate the technology-fueled shift to virtual sexual interactions for many adolescents, in the form of sexting, video chatting, and the use of pornographic websites (Klein \& Cooper, 2019). Some educators, policymakers, parents, and academics have therefore expressed concern about the impacts of exposure and access to pornography on children, adolescents, and youth (see Chapter 4 for an overview of "effects" research on adolescents and youth). ${ }^{2}$

Since the capacities of human brains for reasoning, judgment, and regulating risk-taking behaviors do not fully form until approximately the age of 25 (Blakemore \& Robbins, 2012), some medical practitioners and scholars have expressed concern about the impact of online pornography on adolescent and youth's sexual expectations and experiences (for a review of existing research, see Owen, Behun, Manning, \& Reid, 2012). For example, studies by Peter and Valkenburg concluded that adolescents-particularly those who frequently use pornography - may be more likely to believe that pornography represents sexual reality (Peter \& Valkenburg, 2006, 2011). These findings, however, have been challenged by Löfgren-Mårtenson and Månson (2010), who found that most adolescent participants were able to distinguish between pornography and real-life sexual interactions, although participants did note the influence of pornographic scripts on their sexual practices.

\section{The mainstreaming of BDSM}

In line with the broader sociocultural mainstreaming of pornography described above, over the last few years BDSM practices have also entered the mainstream (Langdridge \& Barker, 2013; Saunders, 2018). BDSM is an abbreviation for bondage and discipline, dominance and submission, and sadism and masochism (Green, 2015). It is "a group of behaviours and lifestyle practices that include a variety of fetishes, role-playing, and other nonmainstream activities" (Stockwell, Walker, \& Eshleman, 2010, p. 309). Importantly, BDSM pornography is clearly distinguishable from other genres such as "forced sex" (Wilkinson, 2011), given its portrayal of voluntary and clearly consensual exchanges of domination and submission between two consenting participants (Barker, 2013; Ogas \& Gaddam, 2011). The process of BDSM mainstreaming correlates roughly with the release of E.L. James' (2012) trilogy Fifty Shades of 
Grey (Tomazos, O'Gorman, \& MacLaren, 2017), which sold more than 100 million copies worldwide (Green, 2015). The Fifty Shades enterprise has increased both the demand for and the supply of similarly themed pornography. Since the release of the Fifty Shades books, and later on the films, searches for bondage-themed pornography in the UK have increased by 28\% (Pegg, 2018) and various producers have recreated its storyline (Stevens, 2014). Pegg (2018) suggests that such increases indicate a global cultural shift toward the normalization of BDSM.

This mainstreaming of BDSM concepts and practices has been problematized by both members of the BDSM community and pornography scholars. According to experienced BDSM practitioners, healthy and ethical means of consensually combining sex, pain, and aggression "require self-knowledge, communication skills, and emotional maturity," which are present in neither Fifty Shades (Green, 2015; Pfeuffer, 2018) nor in much of the BDSMthemed mainstream pornography (see Makin \& Morczek, 2016; Saunders, 2018). BDSM practitioners are often frustrated by the conflation of power play and power imbalances (Green, 2015), as well as by the general absence of the community's core values, which foreground consent and communication (Pfeuffer, 2018).

According to Makin and Morczek (2016), mainstream pornographic hubs and streaming sites often contain only brief depictions of BDSM scenarios, focusing on inflictions of pain on vulnerable individuals, and mostly lacking the consent briefing that occurs before and after any sexual activity in most BDSM scenarios. Consequently, they argue, there appears to be a significant correlation between interest in BDSM and interest in rape-oriented pornography. Members of the BDSM community have also noted the lack of explicit consent in BDSM pornography (Barker, 2013).

\section{Conclusion}

In this chapter we provided a brief overview of the mainstream pornography industry, focusing on changes in its media, popularity, and consumption patterns over the last five decades. We first reviewed the historical developments in the pornography industry over the last 50 years and noted its recent mainstreaming and normalization. We then described four major trends that characterize and affect the current mainstream pornography industry. First, more and more women are viewing pornography online and there is a small but growing body of research exploring women's patterns of use, their perceptions of their use, and their perceptions of the industry. In the subsequent empirical chapters, we present data from our interviews with female pornography users, including their views on aggression and violence, thus adding our own original contribution to this important, yet understudied, area of research.

Second, largely due to the growth and popularity of "tube sites," more and more content categorized as amateur or "pro-am" is flooding the market, with potentially significant economic and cultural implications (see Ruberg, 
2016). Our own analyses, presented in the empirical chapters of this book, examine the potential relationship between amateur pornography and aggression. Third, we highlighted the related patterns of youth-oriented pornography (which some deem to be on the rise) and youth's consumption of pornography, fueled by the advent of Internet-equipped mobile devices. We discuss the potential effects of this trend in more detail in Chapter 4. Finally, we noted the increasing popularity of BDSM-themed pornography, in part due to the broader mainstreaming of BDSM-related practices. Our interviews with pornography viewers, which we discuss in subsequent chapters, highlight substantial interest among a significant portion of the viewers with whom we spoke in BDSM-related themes and practices.

These user and content trends are intimately tied to broader trends and shifts, namely technological developments and cultural mainstreaming. Technological advances, particularly in personal mobile devices, contribute to the proliferation and normalization of pornography; in turn, "cultural pornification" may be partly responsible for shaping the increasing interest in youthoriented pornography (see Gill, 2008, 2012; Walker, Makin, \& Morczek, 2016). The almost-ubiquitous shift in interface platforms toward "tube sites," afforded by the Internet, has also expanded the demographics of pornography users beyond heterosexual males to include women, amateur content creators, younger viewers, and more people around the world. Given the relative dearth of research on these subgroups of pornography users, the ways in which their consumption patterns shape and reshape the industry are not yet well understood. The analyses we present in the subsequent chapters thus offer important insights on these consumption patterns and individual preferences.

\section{Notes}

1 Web 2.0 is "an umbrella term for the increasing centrality of social media and user-generated content, such as blogs, wikis, online communities, social networking sites, podcasts and different publishing platforms in and for the internet economy" (Paasonen, 2010, p. 1299).

2 There are some ambiguities in how "adolescent" is defined, with some studies examining the impact of pornography on individuals as young as 10 years old (Ybarra \& Mitchell, 2005) and others as old as 22 years old (Braun-Courville \& Rojas, 2009). However, most studies define "adolescents" as those who are 13-18 years of age (e.g. Hunter, Figueredo, \& Malamuth, 2010; Mesch, 2009; Peter \& Valkenburg, 2006).

\section{References}

Albright, J. (2008). Sex in America online: An exploration of sex, marital status, and sexual identity in internet sex seeking and its impact. Journal of Sex Research, 45(2), 175-186. doi:10.1080/00224490801987481 \.

Ashton, S., McDonald, K., \& Kirkman, M. (2019). Pornography and women's sexual pleasure: Accounts from young women in Australia. Feminism \& Psychology, 1-24 (pre-print publication). doi:10.1177/0959353519833410. 
Attwood, F. (2005). What do people do with porn? Qualitative research into the consumption, use, and experience of pornography and other sexually explicit media. Sexuality and Culture, 9(2), 65-86. doi:10.1007/s12119-005-1008-7.

Attwood, F. (2007). No money shot? Commerce, pornography and new sex taste cultures. Sexualities, 10(4), 451-456. doi:10.1177/1363460707080982.

Barcan, R. (2002). In the raw: 'Home-made' porn and reality genres. Journal of Mundane Behavior, 3(1). Retrieved from http://mundanebehavior.org/ in-the-raw-home-made-pornand-reality-genres.

Barker, M. (2013). Consent is a grey area? A comparison of understandings of consent in 50 Shades of Grey and on the BDSM blogosphere. Sexualities, 16, 896-914. doi:10.1177/1363460713508881.

Barnett, V. L. (2018). 'The most profitable film ever made': Deep Throat (1972), organized crime, and the $\$ 600$ million gross. Porn Studies, 5(2), 131-151, doi:10.1080/ 23268743.2017.1343080.

Bauer, J., Gradus, R., \& Jones, R. (2015). Hot girls wanted. Los Angeles, CA: Two to Tangle Productions.

Blakemore, S. J., \& Robbins, T. W. (2012). Decision-making in the adolescent brain. Nature Neuroscience, 15(9), 1184. doi:10.1038/nn.3177.

Boyle, K. (2011). Producing abuse: Selling the harms of pornography. Women's Studies International Forum, 34(6), 593-602. doi:10.1016/j.wsif.2011.09.002.

Braun, V. (2009). "The women are doing it for themselves": The rhetoric of choice and agency around female genital 'cosmetic surgery'. Australian Feminist Studies, 24(60), 233-249. doi:10.1080/08164640902852449.

Braun-Courville, D. K., \& Rojas, M. (2009). Exposure to sexually explicit web sites and adolescent sexual attitudes and behaviors. Journal of Adolescent Health, 45(2), 156-162. doi:10.1016/j.jadohealth.2008.12.004.

Bronstein, C., \& Strub, W. (2016). Porno chic and the sex wars: American sexual representation in the 1970s. Boston: University of Massachusetts Press.

Campbell, L., \& Kohut, T. (2017). The use and effects of pornography in romantic relationships. Current Opinion in Psychology, 13, 6-10. doi:10.1016/ j.copsyc.2016.03.004.

Chowkhani, K. (2016). Pleasure, bodies, and risk: Women's viewership of pornography in urban India. Porn Studies, 3(4), 443-452. doi:10.1080/23268743.2016.1147374.

Church Gibson, P. (2014). Pornostyle: Sexualized dress and the fracturing of feminism. Fashion Theory, 18(2), 189-206. doi:10.2752/175174114X13890223974588.

Cooper, A. (1998). Sexuality and the internet: Surfing into the new millennium. Cyber Psychology and Behavior, 1, 187-193. doi:10.1089/cpb.1998.1.187.

Coopersmith, J. (1998). Pornography, technology and progress. Icon, 4, 94-125. Retrieved from https://www.jstor.org/stable/23785961

Cowan, J. (2010). Sex isn't selling. Canadian Business, 83(15): 26-30. Retrieved from https://www.canadianbusiness.com/business-strategy/sex-isnt-selling/

de Cadenet, A. (2015, October 19). More women watch (and enjoy) porn than you ever realized: A Marie Claire study. Marie Claire. Retrieved from http://www. marieclaire.com/sex-love/a16474/womenporn-habits-study/

DeLamater, J., \& Plante, R. F. (Eds.). (2015). Handbook of the sociology of sexualities. Dordrecht, Switzerland: Springer.

Dines, G. (2006). The white man's burden: Gonzo pornography and the construction of black masculinity. Yale Journal of Law and Feminism, 18(1), 283-297. Retrieved from https://digitalcommons.law.yale.edu/yjlf/vol18/iss1/12 
Dines, G. (2009). "Childified women: How the mainstream porn industry sells child pornography to men." In S. Olfman (Ed.), The sexualization of childhood. Westport, CT: Praeger.

Dines, G. (2010). Pornland: How porn has hijacked our sexuality. Boston, MA: Beacon Press.

Dines, G. (2012). A feminist response to Weitzer. Violence Against Women, 18(4), 512-520. doi:10.1177/1077801212452550.

Doran, K. (2008). "Industry size, measurement, and social costs (presentation, Princeton University, Princeton, New Jersey, December 11-13, 2008). Retrieved from http://www.socialcostsof pornography.com/Doran_Industry_Size_ Measurement_Social_Costs.pdf)

Effron, L. (2011, October 14). The appeal of amateur porn. ABC News. Retrieved from https://abcnews.go.com/blogs/health/2011/10/14/the-appeal-of-amateur-porn)

Flood, M. (2007). Exposure to pornography among youth in Australia. Journal of Sociology, 43, 45-60. doi:10.1177/1440783307073934.

Forrester, K. (2016, September 19). Making sense of modern pornography. The New Yorker. Retrieved from https://www.newyorker.com/magazine/2016/09/26/ making-sense-of-modern-pornography

Foucault, M. (1992). The perverse implantation. In E. Stein (Ed.), Forms of desire: Sexual orientation and the social constructionist controversy (pp. 11-24). New York, NY: Routledge.

Fradd, M. (2017). The porn myth: Exposing the reality behind the fantasy of pornography. San Francisco, CA: Ignatius Press.

Gilkerson, L. (2013, November 14). Rape for profit: New documentary shows the faces of sex trafficking." Retrieved from https://www.covenanteyes.com/2013/11/14/ rape-profit-new-documentary-shows-faces-sexual-exploitation/

Gill,R. (2008).Empowerment/sexism:Figuring female sexualagencyincontemporary advertising. Feminism \& Psychology, 18(1), 35-60. doi:10.1177/0959353507084950.

Gill, R. (2012). The sexualisation of culture?. Social and Personality Psychology Compass, 6(7), 483-498. doi:10.1111/j.1751-9004.2012.00433.x.

Green, E. (2015, February 10). Consent isn't enough: The troubling sex of Fifty Shades. Retrieved from https://www.theatlantic.com/entertainment/archive/2015/02/ consent-isnt-enough-in-fifty-shades-of-grey/385267/

Grey, A. (2015, September 28). You've heard of rape culture, but have you heard of pedophile culture? Retrieved from https://www.feministcurrent.com/2015/09/28/ youve-heard-of-rape-culture-but-have-you-heard-of-pedophile-culture/

Hald, G. M., Malamuth, N. M., \& Lange, T. (2013). Pornography and sexist attitudes among heterosexuals. Journal of Communication, 63(4), 638-660. doi:10.1111/ jcom.12037.

Hald, G. M., Seaman, C., \& Linz, D. (2014). Sexuality and pornography. In D. L. Tolman \& L. M. Diamond (Eds.), APA handbook for sexuality and psychology: Volume 2. Contextual approaches (pp. 3-35). Washington, DC: American Psychological Association.

Harrison, L., \& Ollis, D. (2015). Young people, pleasure, and the normalization of pornography: Sexual health and well-being in a time of proliferation?. In J. Wyn \& $\mathrm{H}$. Cahill (Eds.), Handbook of children and youth studies (pp. 155-167). Singapore: Springer.

Head, T. (2019). The history of pornography. Retrieved from https://www. thoughtco.com/history-of-pornography-721217

Huffpost. (2017, June 6). Porn sites get more visitors each month than Netflix, Amazon and Twitter combined. Retrieved from https://www.huffingtonpost. ca/2013/05/03/internet-porn-stats_n_3187682.html?ri18n=true 
Hunter, J. A., Figueredo, A. J., Malamuth, N. M., \& Becker, J. V. (2003). Juvenile sex offenders: Toward the development of a typology. Sexual Abuse: A Journal of Research and Treatment, 15(1), 27-48. doi:10.1023/A:1020663723593.

Jacobs, K. (2007). Netporn: DIY web culture and sexual politics. Lanham, MD: Rowman and Littlefield Publishers.

James, E. L. (2012). Fifty Shades of Grey. London, UK: Century.

Jensen, R. (2007). Getting off: Pornography and the end of masculinity. Cambridge, MA: South End Press.

Jensen, R. (2011). Stories of a rape culture: Pornography as propaganda. In M. Tankard Reist, \& A. Bray (Eds.), Big Porn Inc.: exposing the harms of the global pornography industry (pp. 25-33), North Melbourne, Vic: Spinifex Press.

Kendrick, W. M. (1997). The secret museum: Pornography in modern culture. Berkeley: University of California Press.

Klein, J. L., \& Cooper, D. T. (2019). Deviant cyber-sexual activities in young adults: Exploring prevalence and predictions using in-person sexual activities and social learning theory. Archives of Sexual Behavior, 48(2), 619-630. doi:10.1007/ s10508-018-1251-2.

Konow, D. (2007). Technology meets porn. Retrieved from https://www.tomshardware. co.uk/ technology-porn-review-29590-2.html

Langdridge, D., \& Barker, M. (2013). Sadomasochism: Past, present, future. In D. Langdridge, C. Richards, \& M. J. Barker (Eds.), Safe, sane and consensual: Contemporary perspectives on sadomasochism (pp. 3-13). London, UK: Palgrave Macmillan.

Lehman, P. (2003). Bad: Infamy, darkness, evil, and slime on screen. Albany, NY: State University of New York Press, 79-88.

Lewis, L., Somers, J. M., Guy, R., Watchirs-Smith, L., \& Skinner, S. R. (2018). 'I see it everywhere': Young Australians' unintended exposure to sexual content online. Sexual Health, 15(4), 335-341. doi:10.1071/SH17132.

Levy, D., \& Dines, G. (13 November 2013). Good cop bad cop: Corporate political strategy in the porn industry. Retrieved from https:// organizationsandsocialchange.wordpress.com/2013/11/13/good-cop-bad-cop-corporatepolitical-strategy-in-the-porn-industry/

Lo, V. H., \& Wei, R. (2005). Exposure to internet pornography and Taiwanese adolescents sexual attitudes and behavior. Journal of Broadcasting and Electronic Media, 49(2), 221-237. doi:10.1207/s15506878jobem4902_5.

Lodder, M. (2016). Visual pleasure and gonzo pornography: Mason's challenge to convention in 'the hardest of hardcore'. Porn Studies, 3(4), 373-385. doi:10.1080/ 23268743.2016.1241160.

Löfgren-Mårtenson, L., \& Månsson, S. A. (2010). Lust, love, and life: A qualitative study of Swedish adolescents' perceptions and experiences with pornography. Journal of Sex Research, 47(6), 568-579. doi:10.1080/00224490903151374.

Long, J. (2016). Pornography is more than just sexual fantasy. It's cultural violence. In Theory: Opinion. Retrieved from https://www.washingtonpost.com/ news/in-theory/wp/2016/05/27/pornography-is-more-than-just-sexual-fantasyits-cultural-violence/?noredirect $=$ on

Makin, D. A., \& Morczek, A. L. (2016). X views and counting: Interest in rapeoriented pornography as gendered microaggression. Journal of Interpersonal Violence, 31(12), 2131-2155. doi:10.1177/0886260515573572.

Martinez, M., \& Manolovitz, T. (2010). Pornography of gaming. In D. Riha (Ed.), Videogame cultures and the future of interactive entertainment (pp. 65-74). Oxford, UK: Inter-Disciplinary Press. 
McIntyre, S., \& Clark, D. (2015). The role of technology in human trafficking. A white paper prepared for Microsoft. Retrieved from http://mtroyal.ca/cs/ groups/public/documents/pdf/ humantrafficking.pdf

McKee, A., Albury, K., \& Lumby, C. (2008). The porn report. Victoria, Australia: Melbourne University Press.

McKeown, J. K., Parry, D. C., \& Light, T. P. (2018). "My iPhone changed my life": How digital technologies can enable women's consumption of online sexually explicit materials. Sexuality and Culture, 22(2), 340-354. doi:10.1007/ s12119-017-9476-0.

McNair, B. (2002). Striptease culture: Sex, media and the democratisation of desire. London, UK: Routledge.

McNair, B. (2013). Porno? Chic! How pornography changed the world and made it a Better Place. New York, NY: Routledge.

McNeil, L., Osborne, J., \& Pavia, P. (2005). The other Hollywood: The uncensored oral history of the porn film industry. New York, NY: HarperCollins.

Mercer, J. (2004). In the slammer: The myth of the prison in American gay pornographic video. Journal of Homosexuality, 47(3-4), 151-166. doi:10.1300/ J082v47n03_08.

Miller-Young, M. (2007). Sexy and smart: Black women and the politics of selfauthorship in netporn. In K. Jacobs, M. Janssen, \& M. Pasquinelli (Eds.), C'LickMe: A Netporn Studies Reader (pp. 205-216). Amsterdam, Netherlands: Institute of Network Cultures.

Miklos, J. R., \& Moore, R. D. (2008). Labiaplasty of the labia minora: Patients' indications for pursuing surgery. The Journal of Sexual Medicine, 5(6), 1492-1495. doi:10.1111/j.1743-6109.2008.00813.x.

Morichetta, A., Trevisan, M., \& Vassio, L. (2019, March). Characterizing web pornography consumption from passive measurements. In International Conference on Passive and Active Network Measurement (pp. 304-316). Springer, Cham, Switzerland.

Ogas, O., \& Gaddam, S. (2011). A billion wicked thoughts: What the internet tells us about sexual relationships. New York, NY: Plume.

Owens, E. W., Behun, R. J., Manning, J. C., \& Reid, R. C. (2012). The impact of internet pornography on adolescents: A review of the research. Sexual Addiction $\mathcal{E}$ Compulsivity, 19(1-2), 99-122. doi:10.1080/10720162.2012.660431.

Paasonen, S. (2010). Labors of love: Netporn, Web 2.0 and the meanings of amateurism. New Media \& Society, 12(8), 1297-1312. doi:10.1177/1461444810362853.

Paasonen, S., Nikunen, K., \& Saarenmaa, L. (2007). Pornification and the education of desire. In S. Paasonen, K. Nikunen, \& L. Saarenmaa (Eds.), Pornification: Sex and sexuality in media culture (pp. 1-20). Oxford, UK: Berg.

Paul, P. (2005). Pornified. New York, NY: Holt.

Paul, P. (2010). From pornography to porno to porn: How porn became the norm. In J. R. Stoner, Jr. \& D. M. Hughes (Eds.), The social costs of pornography: A collection of papers (pp. 3-20). Princeton, NJ: Witherspoon Institute.

Pegg, S. (15 January 2016). Could your taste for 'teen' porn land you in legal trouble? The Conversation. Retrieved from https://theconversation.com/ could-your-taste-for-teen-porn-land-you-in-legal-trouble-53179.

Pegg, S. (16 January, 2018). What the latest data reveals about our passion for pornographyand its legality. The Conversation. Retrieved from http://theconversation.com/whatthe-latest-data-reveals-about-our-passion-for-pornography-and-its-legality- 87817 
Penley, C. (2004). Crackers and whackers: The white trashing of porn. In L. Williams (Ed.), Porn studies (pp. 309-331). Durham, NC: Duke University Press.

Penley, C., Shimizu, C. P., Miller-Young, M., \& Taormino, T. (2013). The feminist porn book: The politics of producing pleasure. New York. NY: The Feminist Press at CUNY.

Peter, J., \& Valkenburg, P. M. (2006). Adolescents' exposure to sexually explicit internet material and recreational attitudes towards sex. Journal of Communication, 56(4), 639-660. doi:10.1111/j.1460-2466.2006.00313.x.

Peter, J., \& Valkenburg, P. M. (2011). The influence of sexually explicit internet material and peers on stereotypical beliefs about women's sexual roles: Similarities and differences between adolescents and adults. Cyberpsychology, Behavior, and Social Networking, 14(9), 511-517. doi:10.1089/cyber.2010.0189.

Pfeuffer, C. (2018, May 17). Fifty shades versus BDSM: The reality of consent. Retrieved from https://www.theglobeandmail.com/life/relationships/ valentines-day/fifty-shades-versus-bdsm-the-reality-of-consent/article33876672/

PornHub. (2015). 2014 year in review. Retrieved from https://www.pornhub.com/ insights/2014-year-in-review

PornHub. (2018). 2018 year in review. Retrieved from https://www.pornhub.com/ insights/2018-year-in-review

PornHub. (2019). The 2019 year in review. Retrieved from https://www.PornHub. com/insights/2019-year-in-review

Ritzer, G., \& Jurgenson, N. (2010). Production, consumption, prosumption: The nature of capitalism in the age of the digital 'prosumer'. Journal of Consumer Culture, 10(1), 13-36. doi:10.1177/1469540509354673.

Ruberg, B. (2016). Doing it for free: Digital labour and the fantasy of amateur online pornography. Porn Studies, 3(2), 147-159. doi:10.1080/23268743.2016.1184477.

Sanghani, R. (20 October 2014). Designer vaginas: Why young women are searching for porn star perfection. Retrieved from https://www.telegraph.co.uk/women/ womens-health/11172674/Designer-vaginas-Why-young-women-are-searchingfor-porn-star-perfection.html

Saunders, R. (2018). Grey, gonzo and the grotesque: The legacy of porn star Sasha Grey. Porn Studies, 5(4), 363-379. doi:10.1080/23268743.2018.1505544.

Schwyzer, H. (2012). Why do men love 'barely legal' porn? Jezebel. Retrieved from https://jezebel.com/why-do-men-love-barely-legal-porn-5881335)

Shimizu, C. P. (2010). Screening sexual slavery? Southeast Asian Gonzo Porn and US Antitrafficking Law. Sexualities, 13(2), 161-170. doi:10.1177/1363460709359230.

Smits, K. (2016). Applying political theory: Issues and debates (2nd ed.). New York, NY: Palgrave.

Snape, T. D. (2010). "Girls don't watch porn”: A narrative analysis of women's negotiations with 'problematic spaces'. Unpublished master's thesis. Kelowna: University of British Columbia Okanagan.

Stella, R. (2016). The amateur roots of gonzo pornography. Porn Studies, 3(4), 351361. doi:10.1080/23268743.2016.1241157.

Stevens, S. (2014). Rope sluts, and bottoms, and subs, oh my: 50 shades of grey and the shifting discourse on female submission in feminist kink porn. The Communication Review, 17, 256-268. doi:10.1080/10714421.2014.930602.

Stockwell, F. M. J., Walker, D. J., \& Eshleman, J. W. (2010). Measures of implicit and explicit attitudes toward mainstream and BDSM sexual terms using the IRAP and questionnaire with BDSM/Fetish and student participants. The Psychological Review, 60(2), 307-324. doi:10.1007/BF03395709. 
Taormino, T., Parrenas, C., Shimizu, C., \& Miller-Young, M. (2013). The feminist porn book: The politics of producing pleasure. New York, NY: The Feminist Press at CUNY.

Tarrant, S. (2016). The pornography industry: What everyone needs to know. Oxford, UK: Oxford University Press.

Tomazos, K., O'Gorman, K., \& MacLaren, A. (2017). From leisure to tourism: How BDSM demonstrates the transition of deviant pursuits to mainstream products. Tourism Management, 60, 30-41. doi:10.1016/j.tourman.2016.10.018.

Van Doorn, N. (2010). Keeping it real: User-generated pornography, gender reification, and visual pleasure. Convergence: The International Journal of Research into New Media Technologies, 16(4), 411-430. doi:10.1177/1354856510375144.

Walker, A., Makin, D. A., \& Morczek, A. L. (2016). Finding Lolita: A comparative analysis of interest in youth-oriented pornography. Sexuality $\&$ Culture, 20(3), 657-683. doi:10.1007/s12119-016-9355-0

Weinberg, M. S., Williams, C. J., Kleiner, S., \& Irizarry, Y. (2010). Pornography, normalization, and empowerment. Archives of Sexual Behavior, 39(6), 1389-1401. doi:10.1007/s10508-009-9592-5.

Wilkinson, E. (2011). "Extreme pornography" and the contested spaces of virtual citizenship. Social \& Cultural Geography, 12(5), 493-508. doi:10.1080/14649365. 2011.589535.

Wilkinson, E. (2017). The diverse economies of online pornography: From paranoid readings to post-capitalist futures. Sexualities, 20(8), 981-998. doi:10.1177/1363460716675141.

Wolak, J., Mitchell, K., \& Finkelhor, D. (2007). Unwanted and wanted exposure to online pornography in a national sample of youth Internet users. Pediatrics, 119(2), 247-257. doi:10.1542/peds.2006-1891.

Wosick, K. (2015). Pornography. In J. DeLamater \& R. F. Plante (Eds.), Handbook of the sociology of sexualities (pp. 413-434). Dordrecht, Switzerland: Springer.

Ybarra, M. L., \& Mitchell, K. J. (2005). Exposure to Internet pornography among children and adolescents: A national survey. Cyberpsychology \& Behavior, 8(5), 473-486. doi:10.1089/cpb.2005.8.473. 


\section{Research methodology and definitions}

In Chapters 4 through 7, we present results from a large-scale study of free online pornographic videos and viewers reactions to these videos. Our study design includes two major components: A content analysis of 409 online pornographic videos and 121 interviews with individuals who regularly watch pornography online. These two study components complement one another and assist us in achieving a more complete and multifaceted picture of today's pornography industry and the role of aggression within this industry.

\section{Research component 1: content analysis of online pornographic videos}

The first component of our empirical research is designed to address questions concerning the overall prevalence of aggression in online mainstream pornography as well as time-trends in this prevalence. We also sought to explore variations in aggression by race/ethnicity and by the gender composition of the performers. Similar to previous research in the field, we therefore used content analysis of frequently watched pornographic videos uploaded to a free online content-sharing website (PornHub). Our sampling strategy was designed to capture a wide variety of videos, including a significant range of years, variability in popularity of videos, and variability in the racial and gender compositions of performers.

\section{Sampling strategy and procedures}

\section{Selection of website: PornHub}

We coded 409 videos from PornHub, one of the world's top adult websites, and according to Alexa Internet, the 36th most visited site on the Internet as of 2017, with more than 80 million daily visits (PornHub, 2018). PornHub is a freely accessible sharing website similar to YouTube. While some former studies of Internet pornography examined multiple websites (e.g. Klaassen \& Peter, 2015; Vannier, Currie, \& O’Sullivan, 2014), many of the most popular websites have overlapping ownerships (van der Linde, 2016). Therefore, the list of most watched videos in these websites includes multiple overlapping and consequently comparable videos (Klaassen \& Peter, 2015). We also chose to focus on PornHub because this site allows certain types of specified searches, 
which were theoretically interesting for us. These include searching for random videos from within the entire set of videos on the website (see below) and options for identifying the year in which videos were uploaded to the website.

Our choice to use PornHub as the source of videos for our analysis was further supported by the viewing preferences reported by our interviewees (see Research Component 2 below), as more than $90 \%$ of them reported watching pornography primarily in PornHub or a very similar free porn video-sharing service. About $80 \%$ of our interviewees watched porn through one of the free porn video-sharing services that are owned by MindGeek, such as PornHub, YouPorn, and RedTube. Of these, more than 90\% identified PornHub as their only or primary source of porn consumption. An additional 13\% of the overall sample of interviewees watched pornography primarily through another free porn video-sharing service, which is not owned by MindGeek but looks very much like PornHub and includes very similar materials. These websites are typically owned by the other big player in the free porn video-sharing industry, WGCZ, and include XVideos and XNXX. Finally, $5 \%$ of the sample watched pornography primarily through the free social networks and news aggregation websites Tumblr and Reddit. Only four interviewees reported sometimes paying for pornography online. All four were women who watched pornography primarily using Bellesa, a pornographic video-sharing platform that is woman-friendly and produced by women.

\section{Strategy for sampling videos within PornHub}

We conducted our sampling in four distinct stages, designed to help us explore the various questions we had about the content of videos in PornHub. These included (1) a sample of the "all time most watched" videos; (2) a sample of "random" videos; (3) a quota sample of videos from a few selected racially diverse categories; and (4) a quota sample of same-sex videos. Overall, we analyzed 409 videos in this study. All these videos were uploaded to PornHub between 2008 and 2016. Table 3.1 includes additional details on the size of each of these samples, the particular analyses for which they were utilized, and the source of the sample. Below we provide additional details on each of these samples.

I The "all time most watched" sample $(n=70)$. First, similar to other recent analyses (Bridges, Wosnitzer, Scharrer, Sun, \& Liberman, 2010; Klaassen \& Peter, 2015; McKee, 2005), we sought to analyze the most watched videos from each of our predefined categories. This strategy was designed to increase generalizability and explore the porn content that is most likely to be watched by wide audiences (and therefore have a potentially larger cultural impact). Hence, all the videos in this part of the sample received at least two million visits. All 70 videos in this sample included two partners (we excluded from the sample three videos that did not include two partners).

II The "random" sample $(n=76)$. To complement the sample of most-watched videos and in order to analyze a comparative sample of videos that were 


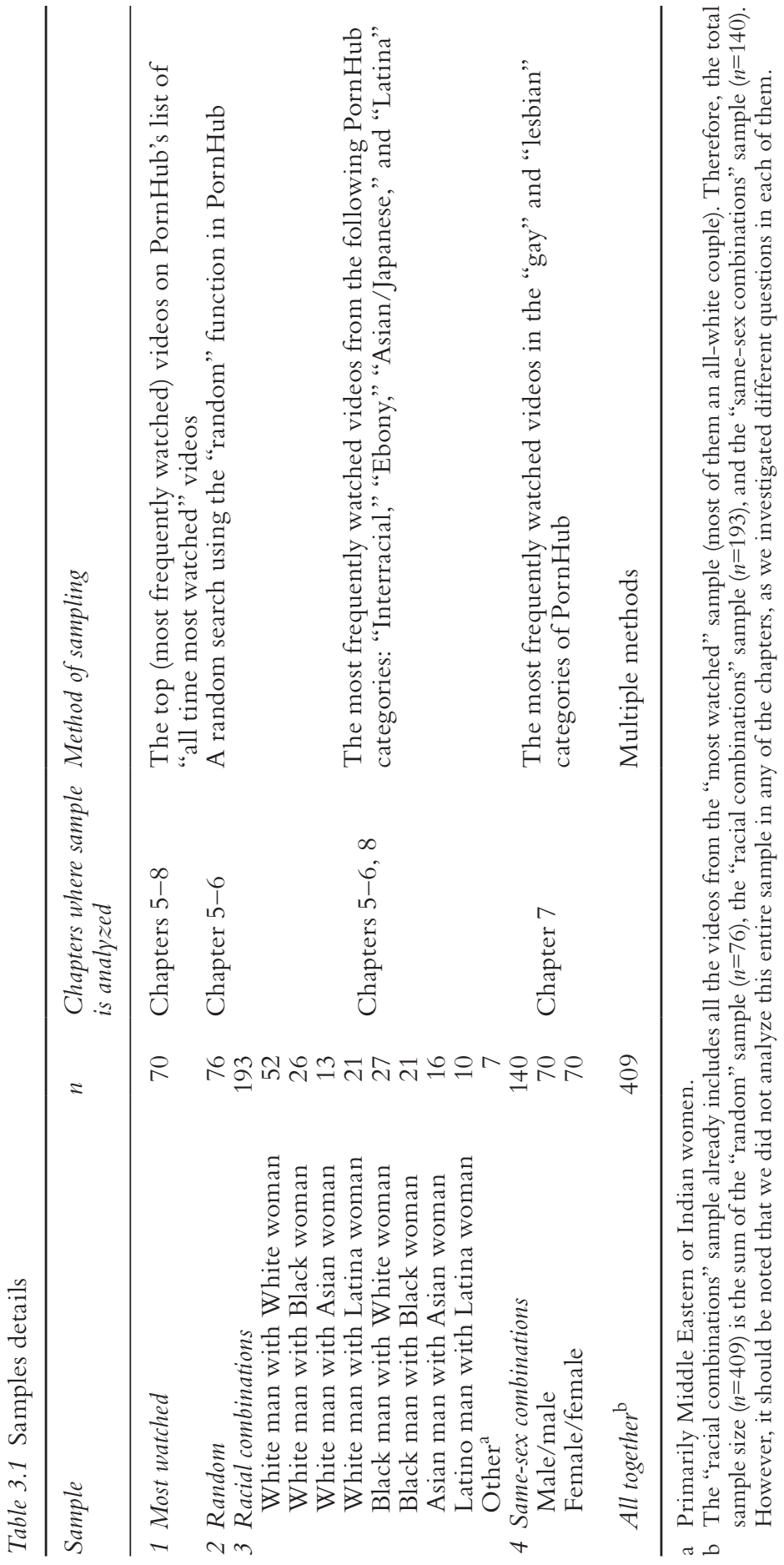


less popular, we also retrieved a random sample of videos using PornHub's "Random" function, which randomly samples a video from the website's archives. We first sampled 80 videos and then excluded four of them that did not include a couple of a man and a woman, resulting in a sample size of 76 videos. It should be noted that we did not use this sample as a comparison in the analyses that examine racially diverse videos and same-sex videos, since PornHub does not allow a random search within these categories.

III Quota sampling for racial/ethnic diversity $(n=123)$. Most previous content analyses of pornography either examined the most watched/most rented videos (Bridges et al., 2010; Klaassen \& Peter, 2015; McKee, 2005) or they used convenience or random sampling techniques (Gorman et al., 2010; MonkTurner \& Purcell, 1999). Consequently, these studies were not able to reach substantial variation in race and ethnicity and most of them did not analyze minorities in pornography. Seeking to achieve such ethno-racial variability, we chose to employ a purposive sampling technique. Most of the videos on the "all-time most watched" sample included sexual interactions between a White (North American) man and a White (North American) woman. We therefore purposively sampled additional all-time most-watched videos from each of the following PornHub categories: "Interracial" (25 videos), "Ebony" (52 videos), "Asian/Japanese" (35 videos), and "Latina" (19 videos). This strategy was designed to reach at least 25 videos that include White women with non-White men, Black women, Asian women, and Latina women (when combined with the all-time most-watched videos). After excluding videos that did not include men (four videos) and videos containing more than two participants (five videos), we reached a sample of 123 videos. This sample included at least 25 videos for each of the following racial/ethnic combinations: a White man with a Black woman, a Black man with a White woman, a Black man with a Black woman, an Asian woman (with any partner), and a Latina woman (with any partner). Other racial combinations (e.g. an Asian man with a White woman or a Latino man with a Black woman) were not accessible through PornHub categories.

IV Quota sampling for same-sex videos $(n=140)$. Our final sample was designed to allow us to contrast the videos from the general all-time most-watched sample (all including a dyad of a man and a woman) with comparable same-sex videos. We therefore sampled 70 videos from the all-time most frequently watched videos in the "gay" category of PornHub (all including a dyad of two men) and an additional 70 videos from the all-time most frequently watched videos in the "lesbian" category (all including a dyad of two women).

\section{Measurements and coding}

\section{Defining and coding aggression/violence and affection/pleasure displays}

Definitional ambiguities regarding violence and aggression in pornography have historically plagued both public and academic discussions the field and 
have been a source of ongoing contention. Violence has often been defined simply as behaviors directed from one person to another that are intended to cause harm (Mellor \& Duff, 2019). McKee (2005, 2015), however, has argued that while most former analyses of sexually explicit materials (SEM) have dismissed consent in their definition of aggression. This omission, in turn, leads to problems in understanding the relationship between SEM and healthy sexual development. He therefore advocated for a careful inspection of the interactions in SEM, treating consent as an ongoing process, which considers the entirety of the sexual interaction and accounts for both physical and verbal expressions of consent. Similarly, Ramirez (2009) noted an important distinction between two types of aggression-hostile and instrumental. While the former is aggression conducted with the intention to harm, instrumental aggression is used to achieve a goal, such as exerting control or at times eliciting pleasure (e.g. in pornography that includes bondage, discipline, sadism, and masochism (BDSM)).

These insights about the significance of consent and instrumental aggression are important for our understanding, interpretation, and coding of aggression in pornography. However, the majority of former content analyses in this field have used a much broader definition of aggression, one that does not focus on consent (Barron \& Kimmel, 2000; Bridges et al., 2010; Cowan \& Campbell, 1994; Cowan et al., 1988; Gorman et al., 2010; Klaassen \& Peter, 2015; Malamuth \& Spinner, 1980; Monk-Turner \& Purcell, 1999; Scott \& Cuvelier, 1993). Bridges et al. (2010) summarized the rationale for employing a broader and more inclusive definition, claiming that the genre of pornography often requires performers to express enjoyment following just about any act or situation. Considering these powerful expectations, they argued, almost no act would be coded as aggressive under a definition that requires a target to clearly show displeasure, rendering sexual aggression invisible. Bridges et al. (2010) therefore defined aggression as "any purposeful action causing physical or psychological harm to oneself or another person, whereby psychological harm is understood as assaulting another verbally or nonverbally" (p. 1072).

Indeed, it is often hard for viewers of SEM to distinguish between "authentic" and "performed" consent. In addition, aggression or degradation are often a matter of perception and subjectivity: what one individual may consider degrading or aggressive another may consider kinky or arousing (Tarrant, 2016). Still, we hold that even if consent is not authentic and some viewers may be aware of this fact, the performance itself carries substantial importance, as it prescribes a normative sexual script where aggression is acceptable only (or mostly) when it is performed with the consent of the receiving party.

Recognizing the merits of both the approaches to aggression described above, in the present study we adopted two different operational measurements of aggression. The first, in line with Bridges et al. (2010) and most other previous studies, focused on the acts themselves and on the apparent intent to cause harm. Following this definition (henceforth: "visible aggression"), we coded the following acts as physically aggressive: (1) biting, (2) pinching, 
(3) kicking, (4) pulling hair, (5) hitting of the face, (6) hitting of the body, (7) choking, (8) forced gagging, (9) spanking, (10) sadomasochism, (11) rough handling (e.g. pushing, shoving, tossing, shaking), and (12) forceful penetration (vaginal or anal) with penis, hand, or another object, with an apparent intent to cause pain/discomfort. We also noted the duration of each of these acts relative to the duration of the entire video.

Next, in line with McKee's (2015) conceptualization of aggression (henceforth: "non-consensual aggression"), we watched carefully for both verbal and physical cues for lack of consent. These could include either explicit verbal requests to stop or avoid a certain act, nonverbal signs of resistance, attempts to avoid the act, or evident unhappiness at being in the situation or performing a certain act, which were nevertheless ignored by the other party. Whenever such verbal or nonverbal cues appeared, we coded the video as containing non-consensual aggression.

In addition to noting whether a video contained aggression or not, we also coded the total length of aggressive acts and then used this figure to calculate the percentage of the video including visible aggression. This variable is important, as a video that lasts 30 minutes, which includes only two seconds of slapping, would still be considered as containing aggression, but it is clearly not the same as a video in which half of the playing time is devoted to aggressive acts. Next, for each of the videos, we also determined whether the title suggests aggression. Examples of titles suggesting aggression were "Gigantic Cock Rips Skinny Bitch" and "Teeny Booper Kidnapped by Huge Black Cock." Examples of more neutral titles included "Stunning MILF Has the Most Spectacular Tits" and "Office Asian Fuck." While titles may not accurately represent the actual content of videos, we believe that they are often important to analyze in their own right, as even the suggestion of aggression may be an important part of the fantasy and viewing experience. In addition, we also noted the occurrence of verbal aggression, which included practices such as yelling, name-calling, threatening, and swearing at a sexual partner.

Finally, while much of the previous research on pornography focused mainly on aggression and humiliation, it is important to also examine whether and to what extent Internet pornography depicts displays of mutual affection and of pleasure and satisfaction by both partners. We therefore coded the presence and relative length of affectionate acts, including acts such as kissing, hugging, caressing, praising, and sweet-talking. In addition, we noted pleasure responses and displays (e.g. moaning, screaming in pleasure, or clearly showing a climax), particularly by the non-dominant partner in the sexual act.

\section{Coding aggression in same-sex videos}

When coding the dominant and submissive partners roles in "heterosexual" (hereafter "male/female," or "m/f") videos, we assigned "M1" to the male, and "F1" to the female, since all clips employed a heteronormative script that cast women in a submissive and men in a dominant position. All the 
videos in the same-sex samples also had a clear initiator, and most of them also mirrored a dominant/submissive dichotomy, similar to the one found in the heterosexual videos. For the "lesbian" (hereafter "female/female," or "f/f") clips, we coded as "dominant" the woman who initiated sexual activity, expressed aggression (in clips containing aggression), penetrated the other woman (in clips featuring sex toys), or possessed some form of social or economic power (e.g. employer, teacher, or stepmother). For the male/male clips, we coded as "dominant" the man who initiated sexual activity, expressed aggression (in clips containing aggression), or possessed some form of social or embodied power (e.g. larger size). Still, we also coded and analyzed any instances of reverse aggression and "dominating" behavior in all videos: three of the female/female clips featured mutual sex toy penetration, and three of the male/male clips featured mutual anal penetration.

In terms of sexual acts, however, we had to adopt a more sophisticated coding strategy. Certain acts (e.g. cunnilingus) were not relevant for clips featuring two men, and while penetration for $\mathrm{m} / \mathrm{f}$ videos always referred to a flesh penis, in $\mathrm{f} / \mathrm{f}$ clips, penetration referred to a sex toy and/or digits and could have been initiated by either one of the performers. In addition to coding sexual acts, we also collected and thematically coded the "tags" associated with each clip, dividing them into themes such as "descriptions of body parts" (e.g. breasts, penis size), "location" (e.g. couch, locker room), or "sexual acts" (e.g. oral sex). Our main goal in the coding process was to compare aggression against and pleasure displays among women in $\mathrm{m} / \mathrm{f}$ videos to aggression against and pleasure displays of the individuals who assumed a more submissive (non-dominant) role in the $\mathrm{m} / \mathrm{m}$ and $\mathrm{f} / \mathrm{f}$ videos. We should note that although none of the analyzed clips indicated the involvement of trans` performers, we can neither assume nor ascertain that all performers were cisgender.

\section{Coding race/ethnicity}

As described above, our sampling strategy sought to capture a wide variety of racial/ethnic groups and the various interactions among them. While we clearly recognize that racial and ethnic categorizations are social constructions, rather than biological categories, these social constructions have a significant impact on social realities. In other words, the ways in which viewers perceive the race or ethnicity of performers and the typical stereotypes associated with them affect the perception and often also the treatment of the corresponding ethnic and racial groups in everyday life.

We used the following cues in determining performers' race/ethnicity: (1) the titles of videos, (2) the categories in which videos were classified (e.g. "ebony”), (3) the tags awarded to each video (e.g. "Asian," "Ebony," "Czech," "Brunette," and "Latina"), and (4) the physical appearance of performers (in cases where the video did not otherwise indicate race or ethnicity). The first three indicators (title, category, and tag) are important because even if the performer is not actually what the title or tag suggest (e.g., a "Muslim 
Middle-Eastern" who is actually portrayed by a Caucasian American-born woman), the title, category, and tag serve as an important part of the fantasy marketed to viewers.

We were able to categorize all performers into one of the following racial/ ethnic categories: Caucasian American, Caucasian European, light-skinned Middle Eastern, dark-skinned Middle Eastern, Black (Afro-American), bi-racial or light-skinned Black, Latino/Latina, South-East Asian, and East Asian. Since many of these initial categories included very few observations, we collapsed them in the final analyses into four major categories: White (including light-skinned Middle Eastern), Black (including light-skinned Black), Latino/Latina, and Asian.

\section{Coding procedures and inter-coder reliability}

Two female coders worked on this project. Both were graduate students with good familiarity of sexually explicit materials from previous research projects. They both coded all the videos in the sample separately and then met to compare their coding. The coders first met several times with the project leader (the lead author), who trained them in the method and coding scheme. Before coding from the actual sample, each of them coded five trial videos separately. The research team then met to discuss the coding and resolve unclear issues. Next, the coders continued to code all the videos in the sample. When they encountered disagreements over the interpretation of contents, they met with the project leader and the entire research team discussed the issues and reached a resolution. Finally, the project leader watched all the videos to ascertain coding accuracy, paying special attention to the coding of aggression. Differences in coding were then resolved through discussion between the members of the research team.

Inter-coder agreement between students' coding and the project leader's coding was high, with $95.11 \%$ agreement for aggressive titles, $89.24 \%$ agreement for visible aggression, and $96.58 \%$ agreement for non-consensual aggression. Kappa statistic scores for these three key measures were 0.74, 0.78 , and 0.82 respectively, all considered indicators of strong or excellent agreement. The only measurement of aggression for which inter-coder agreement was somewhat lower was the percent of the video containing aggression, with 66.50 inter-coder agreement and a Kappa coefficient of 0.43 , which is often considered fair or moderate. However, we should note that for this variable it is hard to reach a high degree of agreement, as even slight variations in the recording of time (e.g. one minute and a half vs. one minute and 40 seconds) generate disagreement. It is therefore important to note that when disagreements did occur they were usually fairly smallmostly within no more than five seconds difference and never more than 30 seconds difference. When such disagreements did occur, we resolved them by re-watching the video, discussing it, and reaching a consensus agreement. 
While previous studies often used "scene" or "character" as their unit of analysis (Bridges et al., 2010; Cowan \& Campbell, 1994; Klaassen \& Peter, 2015; McKee, 2005), we chose to use the entire video as our unit of analysis, in order to prevent over-representation for longer videos. We should note, however, that most of the videos in our sample only consisted of one sex scene.

\section{Research component 2: interviews with pornography users}

The quantitative component of our analysis is crucial for understanding what is "out there" in the current landscape of free mainstream pornographic videos and what role aggression plays in this market. This analysis examines the videos that viewers are most likely to watch (both overall and in various racial and sexual orientation categories), as well as a random sample of all the videos uploaded to the most popular free online streaming website, thus providing a fairly representative picture of free online mainstream pornography.

Nevertheless, this quantitative content analysis is unable to provide answers to some of the most interesting questions regarding aggression in today's online pornography, namely those that address viewers' views of the industry, preferences, and reactions when they encounter aggression. While the quantitative component of our study does include an analysis of viewers' preferences - in the form of both overall viewership and viewers" "likes" or dislikes"- these measures remain crude, suggestive, and lacking in information. First, they do not provide any information on the reasons for viewers' choices to watch certain videos or their actual judgments of these videos, which may or may not be related to portrayals of aggression. In addition, these measures do not allow us to determine the relationship between the demographic characteristics of viewers and the type of videos they prefer to watch. As such, we are unable to assess whether factors such as gender, age, race, sexual orientation, nationality, and education affect viewers' preferences and approach toward aggression in pornography.

In order to address these important issues, we conducted 100 in-depth interviews with men and women who regularly watch pornographic videos online. We asked interviewees various questions about their watching habits and preferences related to aggression in pornography.

\section{Sampling strategy and recruitment}

Since we were unable to obtain a comprehensive list of online pornography viewers, we had to use a non-probability sampling method. While this method limits generalizability, it is still useful in obtaining rich descriptive data, revealing trends, and generating hypotheses. It is also well-suited for research that seeks to identify and explain highly specific and complex events, processes, preferences, and views (Tansey, 2007). We believe that this method complements our more representative content analysis of videos and helps in providing 
a richer and fuller picture of both the portrayals of aggression in current pornography and viewers' preferences, views, and reactions to these portrayals.

We used a mix of voluntary and purposive sampling to obtain our sample of interviewees. First, in order to obtain our preliminary list of potential interviewees, we advertised both physical and virtual recruitment posters. We posted physical copies of our recruitment poster on bulletin boards in various venues around the City of Montreal, including in coffee shops, public gathering spaces, recreation centers, universities, and community centers. In addition, we posted our recruiting poster to Craigslist, Kijiji, and to several Facebook groups, including a page for recent immigrants to Montreal and multiple student group pages. The poster clarified that potential interviewees must be individuals over the age of 18 who have watched pornographic videos online at least once per month over the previous year. The large majority of interviewees (93\%) reported that they became aware of the study online, through Craigslist, Kijiji, or a Facebook group. ${ }^{1}$ In order to increase participation rates, we also offered each interviewee a $\$ 20$ compensation for participating in the study.

We then applied a theoretically driven purposive sampling strategy, in order to increase variability among our sample of interviewees. Our primary goal in applying this strategy was to be able to explore the relationship between preferences and views on the one hand and various theoretically important factors, such as gender, age, nationality, ethnicity, sexual orientation, and socioeconomic status on the other hand. As such, we sought to reach a roughly balanced number of male and female interviewees (60 each), as well as ensure representation for interviewees from various countries and continents, different sexual orientations, a variety of ethnicities, different ages, and a range of socioeconomic backgrounds and occupations.

Our final list of interviewees does not (and cannot) provide full representation of the population of online pornography viewers. It includes 122 interviewees - 61 who identified as women (one transgender), 60 who identified as men (one transgender), and one who identified as gender-diverse. Although we had interviewees from a wide variety of countries (28 different countries ${ }^{2}$ ) and geographical regions, nearly half of the interviewees were Canadian (45.0\%). The interviewee list also includes a relatively high share of younger people $58.2 \%$ of the interviewees were between the ages of 18 and 25 , though it should be noted that according to PornHub data, more than $60 \%$ of its users are younger than 34$)$, students (71.3\% of all interviewees), and individuals from relatively affluent socioeconomic backgrounds (77.1\%). Any generalizations and conclusions should therefore be done with great caution. Nevertheless, we were able to reach a diverse sample, which captures some of the most theoretically important features and characteristics that may potentially influence viewers' preferences and attitudes toward aggression in pornography.

Table 3.2 presents some of the key descriptive statistics on our sample of interviewees. As noted above, the large majority of our interviewees were 
younger, with the average age of the entire sample slightly lower than 25 years (the women were younger than were the men). Most interviewees were North American, with substantial representation for interviewees from Europe (13.9\%), South Asia (12.3\%), and East Asia (9.0\%). Nearly 80\% of the interviewees identified as heterosexual and the majority of those who did not identified as bisexual (15.6\%). About half of the interviewees (46.7\%) and more than half of the women were in a long-term relationship.

Table 3.2 Descriptive statistics of the interviewees' sample $(n=122)$

\begin{tabular}{|c|c|c|c|}
\hline & $\begin{array}{l}\text { Women } \\
(n=61)\end{array}$ & $\begin{array}{l}\text { Men } \\
(n=60)\end{array}$ & $\begin{array}{l}\text { All } \\
\left(n=122^{a}\right)\end{array}$ \\
\hline Mean number of views per month & 6.8 & 15.3 & 11.4 \\
\hline Mean age & 23.9 & 25.6 & 24.7 \\
\hline Mean age of being introduced to sex & 10.2 & 10.5 & 10.3 \\
\hline $\begin{array}{l}\text { Mean age of first watching pornographic } \\
\text { videos }\end{array}$ & 12.6 & 11.9 & 12.2 \\
\hline \multicolumn{4}{|l|}{ Age categories } \\
\hline 18-19.9 & $14.8 \%$ & $0.0 \%$ & $7.4 \%$ \\
\hline $20-24.9$ & $50.8 \%$ & $51.7 \%$ & $50.8 \%$ \\
\hline $25-29.9$ & $21.3 \%$ & $36.7 \%$ & $29.5 \%$ \\
\hline $30-34.9$ & $6.6 \%$ & $6.7 \%$ & $6.6 \%$ \\
\hline $35-39.9$ & $3.3 \%$ & $1.7 \%$ & $2.5 \%$ \\
\hline $40+$ & $3.3 \%$ & $3.3 \%$ & $3.3 \%$ \\
\hline \multicolumn{4}{|l|}{ Region of residence (at least until age 18) } \\
\hline North America & $55.8 \%$ & $53.3 \%$ & $54.6 \%$ \\
\hline South and Central America & $6.6 \%$ & $5.0 \%$ & $5.7 \%$ \\
\hline Europe & $18.0 \%$ & $10.0 \%$ & $13.9 \%$ \\
\hline Middle East & $0.0 \%$ & $5.0 \%$ & $2.5 \%$ \\
\hline South Asia & $9.8 \%$ & $15.0 \%$ & $12.3 \%$ \\
\hline East Asia & $8.2 \%$ & $10.0 \%$ & $9.0 \%$ \\
\hline Africa & $1.6 \%$ & $1.7 \%$ & $1.7 \%$ \\
\hline \multicolumn{4}{|l|}{ Ethnicity } \\
\hline Caucasian & $59.0 \%$ & $46.7 \%$ & $52.5 \%$ \\
\hline Latin American & $9.8 \%$ & $6.7 \%$ & $8.2 \%$ \\
\hline Middle Eastern & $1.64 \%$ & $6.7 \%$ & $4.1 \%$ \\
\hline East Asian & $13.1 \%$ & $21.7 \%$ & $18.0 \%$ \\
\hline Southwest Asian & $13.1 \%$ & $15.0 \%$ & $13.9 \%$ \\
\hline Indigenous American/Canadian & $0.0 \%$ & $1.7 \%$ & $0.8 \%$ \\
\hline Black & $3.3 \%$ & $1.7 \%$ & $2.5 \%$ \\
\hline \multicolumn{4}{|l|}{ Sexual orientation } \\
\hline Heterosexual & $70.5 \%$ & $85.0 \%$ & $77.1 \%$ \\
\hline Homosexual & $1.6 \%$ & $6.7 \%$ & $4.1 \%$ \\
\hline Bisexual & $24.6 \%$ & $6.7 \%$ & $15.6 \%$ \\
\hline Queer/Pansexual/Sexually fluid & $3.3 \%$ & $1.7 \%$ & $3.3 \%$ \\
\hline
\end{tabular}




\begin{tabular}{lll}
$\begin{array}{l}\text { Women } \\
(n=61)\end{array}$ & $\begin{array}{l}\text { Men } \\
(n=60)\end{array}$ & $\begin{array}{l}\text { All } \\
\left(n=122^{a}\right)\end{array}$ \\
\hline
\end{tabular}

Relationship status

Single

In a relationship

$47.5 \% \quad 58.3 \% \quad 53.3 \%$

$52.5 \% \quad 41.7 \% \quad 46.7 \%$

Socioeconomic background

Low

Medium

High

$\begin{array}{rrr}4.9 \% & 5.0 \% & 4.9 \% \\ 21.3 \% & 15.0 \% & 18.0 \% \\ 73.8 \% & 80.0 \% & 77.1 \%\end{array}$

Education

High school graduate

Undergraduate degree (completed or in process)

Advanced degree (completed or in process)

$\begin{array}{rrr}6.6 \% & 8.3 \% & 7.4 \% \\ 70.5 \% & 71.7 \% & 71.3 \% \\ & & \\ 23.0 \% & 20.0 \% & 21.3 \%\end{array}$

Occupation

Student

Manager

Clerical support

Service and sales

Independent/business owner

Unemployed/not working

$\begin{array}{rrr}70.5 \% & 73.3 \% & 71.3 \% \\ 1.6 \% & 0.0 \% & 0.8 \% \\ 3.3 \% & 1.7 \% & 2.5 \% \\ 14.8 \% & 20.0 \% & 18.0 \% \\ 3.3 \% & 1.7 \% & 2.5 \% \\ 6.6 \% & 3.3 \% & 4.9 \%\end{array}$

a The overall number of interviewees consists of 61 women, 60 men, and one individual who identified as "gender-diverse."

\section{Procedure}

All interviews were conducted in either French or English by two highly skilled and well-trained research assistants. Both interviewers were graduate students in their twenties, a fact that may have helped in establishing rapport and a sense of comfort, as most interviewees in our study were also in their twenties. One of the interviewers was female and the other male, and both interviewed both female and male interviewees. Of note, we could not detect any noticeable differences between interviews conducted by the female and the male interviewers, suggesting that the gender of the interviewers was not significantly affecting interviewees' responses or their level of rapport and comfort in sharing intimate preferences and experiences.

Due to the sensitive nature of the study, and in an effort to increase participation and candid reporting, both the interviewers and the interviewees were encouraged to avoid revealing their real names or any specific identifying details. All interviews were conducted via telephone or Skype audio (without video), in an effort to increase interviewees sense of confidentiality and encourage them to speak candidly about their preferences, experiences, and views. Furthermore, interviewers were careful to reassure interviewees that the interviews were completely private and that no identifying information 
will be disclosed. Indeed, most of the interviewees appeared to be open about their preferences and views and were willing to share them with us without reservations.

Interviews lasted between 30 and 120 minutes. We asked interviewees a series of guiding questions but also allowed them to speak more broadly about other experiences with sexuality and views about pornography. The interviews first featured demographic questions, including age, gender identification, sexual orientation, age, ethnicity, nationality, education, occupation, and relationship status. We then also asked questions about sexual history and history with pornography; questions about their preferences, experiences, and views regarding aggression in pornography; and questions about their preferences, experiences, and views regarding same-sex and different-sex pornography, as well as pornography that includes performers from different ethnicities.

When asking our interviewees more specifically about aggression, we first requested them to tell us which acts they considered to be aggressive and under which circumstances. We then also examined preferences using conventional definitions of aggression in recent content analyses of pornographic content (e.g. Bridges et al., 2010; Klaassen \& Peter, 2015). We thus asked viewers whether they at least sometimes found each of the following 12 items arousing: (1) biting; (2) pinching; (3) kicking; (4) pulling hair; (5) hitting of the face; (6) hitting of the body; (7) choking; (8) forced gagging; (9) spanking; (10) sadomasochism; (11) rough handling; (e.g. pushing, shoving, tossing, shaking); and (12) forceful penetration (vaginal or anal) with penis, hand, or another object, with an apparent intent to cause pain/discomfort. To reduce ambiguity, we asked interviewees to reflect about each of these acts separately.

The female research assistant then entered the responses to questions with a clear answer (e.g. responses to demographic questions and questions with either a positive or a negative response) into a spreadsheet. In addition, she coded binary variables for each of the following six questions regarding aggression (1=yes):

1 Do you at least sometimes enjoy/feel aroused by any acts of aggression in pornography (see list above)?

2 Do you at least sometimes enjoy/feel aroused by any "harder" acts of aggression in pornography (defined as choking, forced gagging, kicking, hitting of the face or body parts, rough handling, and sadomasochism)?

3 Do you sometimes enjoy/feel aroused by non-consensual aggression in pornography?

4 Do you sometimes enjoy/feel aroused by women in pornography expressing pain/discomfort in response to aggression?

5 Would you like to see more aggression in pornographic videos readily available to you?

6 Do you actively search for aggression in pornographic videos? 


\section{Notes}

1 Of note, we did not focus on aggression in our recruitment poster, trying to avoid sampling bias, where individuals with a certain view of aggression (either favorable or unfavorable) would potentially be more likely to participate in the study.

2 We had interviewees from Bangladesh, Belgium, Brazil, Canada, China, Costa Rica, France, India, Indonesia, Iran, Japan, Korea, Lebanon, Mauritius, Mexico, Pakistan, Peru, Romania, Russia, Singapore, Slovenia, Sri Lanka, Syria, the United Kingdom, the United States, Venezuela, Vietnam, and Zimbabwe.

\section{References}

Barron, M., \& Kimmel, M. (2000). Sexual violence in three pornographic media: Toward a sociological explanation. Journal of Sex Research, 37(2), 161-168.

Bridges, A. J., Wosnitzer, R., Scharrer, E., Sun, C., \& Liberman, R. (2010). Aggression and sexual behavior in best-selling pornography videos: A content analysis update. Violence Against Women, 16(10), 1065-1085.

Cowan, G., \& Campbell, R. (1994). Racism and sexim in interracial pornography. Psychology of Women Quarterly, 18, 323-228.

Cowan, G., Lee, C., Levy, D., \& Snyder, D. (1988). Dominance and inequality in X-rated videocassettes. Psychology of Women Quarterly, 12, 299-311.

Gorman, S., Monk-Turner, E., \& Fish, J. N. (2010). Free adult internet Web sites: How prevalent are degrading acts? Gender Issues, 27, 131-145.

Klaassen, M., \& Peter, J. (2015). Gender (iIn)equality in internet pornography: A content analysis of popular pornographic internet videos. Journal of Sex Research, 52(7), 721-735.

Malamuth, N. M., \& Spinner, B. (1980). A longitudinal content analysis of sexual violence in the best-selling erotic magazines. Journal of Sex Research, 16, 226-237.

McKee, A. (2005). The objectification of women in mainstream pornographic videos in Australia. Journal of Sex Research, 42(4), 277-290.

McKee, A. (2015). Methodological issues in defining aggression for content analyses of sexually explicit material. Archives of Sexual Behavior, 44, 81-87.

Mellor, E., \& Duff, S. (2019). The use of pornography and the relationship between pornography exposure and sexual offending in males: A systematic review. Aggression and Violent Behavior, 46, 116-126.

Monk-Turner, E., \& Purcell, C. (1999). Sexual violence in pornography: How prevalent is it? Gender Issues, 17, 58-67.

PornHub. (2018, December 11, 2018). 2018 year in review. Retrieved from https:// www.pornhub.com/insights/2018-year-in-review

Ramirez, J. M. (2009). Some dichotomous classifications of aggression according to its function. Journal of Organisational Transformation and Social Change, 6(2), 85-101.

Scott, J. E., \& Cuvelier, S. J. (1993). Violence and sexual violence in pornography: Is it really increasing? Archives of Sexual Behavior, 22, 357-371.

Tansey, O. (2007). Process tracing and elite interviewing: A case for non-probability sampling. PS: Political Science \& Politics, 40(4), 765-772.

Tarrant, S. (2016). The pornography industry: What everyone needs to know. Oxford, UK: Oxford University Press. 
van der Linde, D. (2016, September 2, 2016). 2016 will be our best year: How the Geeks took over Montreal's porn industry. Retrieved from http://business. financialpost.com/fp-tech-desk/how-the-montreal-porn-industry-has-shiftedfrom-sex-to-tech

Vannier, S. A., Currie, A. B., \& O’Sullivan, L. F. (2014). Schoolgirls and soccer moms: A content analysis of free "Teen" and "MILF" online pornography. Journal of Sex Research, 51(3), 253-264. 


\section{The effects of pornography on consumers}

Pornography's effects have been a matter of fierce debates since the so-called feminist sex wars of the 1980s, which were characterized by public disagreements between anti-porn and sex-positive feminist groups. Beginning in the early 1970s, prominent feminist theorists and activists connected pornography to sexual violence, arguing that pornography almost invariably leads to misogyny and sexual aggression and perhaps even inherently constitutes as violence (e.g. Brownmiller, 1975; Dines, Jensen, \& Russo 1998). Other scholars, however, have been more cautious about the relationship between pornography and violence against women (e.g. Chapkis, 1995; McKee, 2014; Watson \& Smith, 2012; Weitzer, 2011). They have argued that pornography offers a field of multilayered and contradictory contents that can be enjoyed without necessarily causing harm (Chapkis, 1995; McKee, 2014). To date, most research has focused on the potential harmful effects of pornographic materials, although some scholars have also noted neutral or positive ramifications of the growing availability of sexually explicit media (Duggan, Hunter, \& Vance, 1994; McKee, 2014; Watson \& Smith, 2012).

In the first portion of this chapter, we review the debate surrounding the potential harmful effects of pornography. Next, we outline the evidence regarding pornography's effects on gender inequality and sexual violence, looking at both experimental and correlational research designs as well as the various moderating factors in this relationship. We also examine pornography's role as a source of sexual education for adolescents and youth and its part in creating sexual norms and scripts, particularly pertaining to sexual aggression and violence against girls and women. In the final section of this chapter, we bring results from our own interviews with pornography viewers, examining their perceptions regarding the impact of pornography, particularly pornography that includes aggression, on their sexual lives and romantic relationships.

\section{The feminist debates over pornography and its effects}

In the late 1970s and early 1980s, pornography has become a subject of public debate, largely due to tensions between anti-pornography and sex-positive 
feminist activists and scholars. The debate over pornography and its effects was particularly heated among radical and libertarian feminists. The former claimed that pornography leads to the normalization of sexual violence and subsequently to an increase in actual violence, while the latter were concerned that censoring or criminalizing pornography would adversely affect sexual expression for women and sexual minorities (Ferguson, 1984; Lipton, 2012).

Anti-pornography positions hold that pornography is created almost exclusively by men and for men, and thus reflects and sustains male dominance (Dines, 2010). In this view, pornography is intrinsically misogynistic and harmful to women's personal and social well-being (Dworkin, 1981). It portrays women as sexual objects, ready to serve men's sexual needs rather than pursuing mutual pleasure. In the words of feminist activist Robin Morgan, "pornography is the theory, rape is the practice" (Cavalier, 2011). More recently, porn critic Robert Jensen suggested that

pornography is rarely the sole direct causal agent in real-life sexual violence... The discussion should be about the ways in which pornography might be implicated in our rape culture-porn doesn't make men do it, but it's a part of the puzzle.

(Jensen, 2007, p. 102)

This careful proclamation reflects how anti-pornography feminist positions have become more nuanced over the years. Still, over the years, these positions have often shared an uneasy affinity with those of social conservatives (Kaminer, 2013; Whittier, 2014). Although the two groups often differ in their reasons for objecting to pornography, their ultimate goals, and their ties to larger movements (Whittier, 2014), they share a passionate belief in the need to limit the distribution and use of pornography.

Groups who have been most vocally critical of anti-pornography sentiments are often associated with "sex-positive feminism." The term was coined by Ellen Willis, who in a 2005 Village Voice article criticized the antipornography movement. She argued that the movement's claim that "pornography is violence against women" echoes the neo-Victorian idea that "men want sex and women endure it." "Sex-positive" feminists have criticized the anti-pornography movement for repressing sexuality and encouraging censorship, particularly of sexual behaviors that transcend the monogamous, heterosexual norm (McBridge, 2011; Rubin, 1998). Cultural critic Laura Kipnis (1998) challenged the position that porn perpetuates misogyny and sex crimes, criticizing what she saw as the criminalization of fantasy. Anthropologist Gayle Rubin (1984) similarly highlighted the problems with presenting certain sexual practices (e.g. anal sex) or types of sex (e.g. kinky, casual), which fall outside narrow understandings of "healthy sex," as inherently disgusting or debasing. Others have noted that problems such as young men's normalization of sexual aggression or young women's inability to voice their sexual needs precede the widespread availability of online pornography (see 
for example Smith, 2018). Hence, they argue, the perception of pornography as a main cause of violence against women is misguided.

Sex-positive feminists differ in the degree to which they feel pornography is actually beneficial. Libertarian perspectives staunchly maintain that "the benefits pornography provides to women far outweigh any of its disadvantages" (McElroy, 1997, p. 109) and highlight the benefits of "free" sexual expression. Liberal feminist perspectives are more cautious but nevertheless argue that pornography use is generally not harmful unless it contains violence or degradation (Berger, Cottle, \& Searles, 1991). Over the last two decades, alongside the resurgence of anti-pornography feminist arguments (Whisnant \& Stark, 2004), there has also been a parallel rise in more moderate and nuanced arguments about the industry. These latter arguments highlight the difference between causality and correlation in the pornography-aggression association, as well as the importance of distinguishing between types or genres of pornography (Eaton, 2007; Kennedy, Kimmel, \& Llewellyn, 2013; Malamuth, 2018).

\section{How does pornography influence viewers? Sexual script theory}

Pornography has been shown to both reflect and shape attitudes, patterns of desire, and expectations concerning sexuality and gender roles. Simon and Gagnon's (1986) influential sexual script theory has been widely adapted to study pornography's various effects (Wright, Miezan, \& Sun, 2018). The theory, derived from the symbolic interactionist ${ }^{1}$ school of thought (Longmore, 1998), posits that sexuality is a scripted practice, emerging from the interaction of biological factors and sociocultural conditions. Sexual scripts can be understood as personalized cognitive schemata that define sexual reality, behaviors, and preferences (Simon \& Gagnon, 2003). Script theory can therefore help us understand how pornography creates normative expectations for sexual behaviors (Wright, Tokunaga, Kraus, \& Klann, 2017) and how it shapes the preferences of both individuals and social groups (Brown \& L'Engle, 2009), as well as the meanings people attach to sexual behaviors (Hardy, 1998). Pornographic scripts determine what is considered a sexual situation, what event(s) should be included in it, who should participate in it, and how participants should respond (Wright, 2012; Zhou \& Paul, 2016).

Mainstream heterosexual scripts, which dominate mainstream pornography, are largely derived from wider gendered cultural scripts. They prescribe a set of complementary but unequal relational and sexual norms and roles for both women and men. These include the sexual double standard (men want sex and women set sexual limits) and the notion that men have "uncontrollable" sexual needs that ought to be prioritized. By contrast, women should meet men's needs and minimize their own sexual needs (Kim et al., 2007). These scripts are rooted in an understanding of male sexuality as aggressive, insatiable, urgent, and relentless, while female sexuality is perceived as 
passive, cautionary, and discreet (Carpenter, 1998; Durham, 1996). Put otherwise, the mainstream heterosexual script generally mimics normative gender expectations (Mellman, 2017) and constructs two opposing roles: womanas-submissive and man-as-dominant (Courtice \& Shaughnessy, 2018).

Scholars who study media effects and behavioral psychologists have emphasized the need to better understand when and how exposure to and use of media content is related to individuals' attitudes and behaviors. For example, research on learning processes has shown that most people learn more deeply from images (visual learning) than from words (auditory, reading/ writing-based learning) and that people learn better when aroused (Ågmo, Turi, Ellingsen, \& Kaspersen, 2004; Lindner, Blosser, \& Cunigan, 2009). Empirical evidence further suggests that sexual arousal may become conditioned and fantasies can be "primed," based on the pairing of stimuli (e.g. pornographic scenes) and reward (e.g. orgasm) (Brom, Both, Laan, Everaerd, \& Spinhoven, 2014). Finally, some studies have suggested that greater exposure to pornography increases its impact on every day and sexual lives (Peter \& Valkenburg, 2006; Vogels \& O’Sullivan, 2019).

\section{The empirical evidence: pornography's effects on attitudes and behaviors related to sexual aggression, sexual violence, and gender inequality}

Previous studies on the effects of pornography are mostly rooted in behaviorist understandings of sexuality and have generally taken either an experimental or a correlational approach. Experimental studies have relied primarily on lab experiments, while the correlational studies include both studies on sex offenders and studies on non-criminal populations. Below we review the evidence coming from each of these branches. We then examine the research on the various qualifying and moderating factors in the relationship between pornography and real-life aggression, as well as the more specific branch of research that has focused on pornography's effects on attitudes and behaviors in adolescents and youth.

\section{Experimental studies}

Starting in the late 1970s, psychological laboratory studies have attempted to establish a causal relationship between pornography and violent behavior. These studies have focused on the attitudinal and behavioral effects of pornography on samples of largely white, middle-class male undergraduates (Ciclitira, 2004; see Linz and Malamuth, 1993 for an overview). Early studies found that men's exposure to aggressive pornography increased aggressive behaviors in the lab (see Donnerstein, 1980). Early experimental research further showed that even a single exposure to aggressive pornography can result in (1) self-generated rape fantasies (Malamuth, 1981); (2) a less sensitive attitude toward rape (Malamuth \& Check, 1980); and (3) an increased 
acceptance of rape myths and violence against women (Malamuth \& Check, 1981) among male research subjects. Still, other early lab-based findings also found that there was little evidence that non-aggressive pornography had negative impacts. Non-aggressive materials seemed to affect male aggression only following long-term and massive exposure (see Allen, Emmers, Gebhardt, \& Giery, 1995; Donnerstein, 1984).

Early experimental studies have been further plagued by issues of bidirectional causal relationship, whereby "individuals with higher inclinations to aggress against women may derive more gratification from media portrayals of VAW [violence against women] and may also be more susceptible to the influence of such messages" (Malamuth, 1984, p. 40). Consequently, many of these earlier researchers noted the need for research in naturalistic settings with diverse populations (see Malamuth, 1984). More recently, narrative and longitudinal studies have pointed out some other limitations of these laboratory-based studies, particularly their questionable ecological validity (see for example Jensen, 2007). Furthermore, later research has shown that the effects of exposure to aggressive pornography have only been established for certain individuals who were already disposed to sexual aggression and those who share some underlying psychological traits (Hald, Seaman, \& Linz, 2014; Kingston, Malamuth, Fedoroff, \& Marshall, 2009; Malamuth, Hald, \& Koss, 2012; Seto, Maric, \& Barbaree, 2001). We discuss these qualifying studies in more detail below, in the sub-section that addresses the moderating factors in this relationship.

\section{Correlational studies}

Studies on the correlation between pornography and sexual aggression can be divided into two major streams (Malamuth, 2018). The first stream focuses on pornography consumption and criminal acts. It examines testimonies of victims and of sexual offenders regarding pornography-viewing habits and influences; recorded differences in pornography consumption between criminals convicted of sex-related crimes and non-criminals (see Allen, D’Alessio, \& Emmers-Sommer, 1999; Bauserman, 1996); and examinations of the relationship between cross-national aggregate-level consumption of pornography and rape statistics (Diamond, 2009; Diamond, Jozifkova, \& Weiss, 2011; Kutchinsky, 1991). The second stream examines differences among non-criminal populations related to the impact of pornography use on both attitudes and behaviors (see Wright, Sun, Steffen, \& Tokunaga, 2015).

We focus here on the latter subset of studies (those looking at non-criminal populations), as we are mostly interested in pornography's effects on sexual and gendered violence and aggression in the general population. The empirical research examining the relationship between general use of mainstream pornography and sexual- and gender-based violence has produced inconclusive findings. While some studies suggest a relationship between pornography consumption and sexually aggressive behaviors (Peter \& Valkenburg, 2016; 
Wright et al., 2015), others have reported that pornography use is not associated with negative views toward women (McKee, 2014) and argue that the relationship between viewing sexually explicit materials and real-life violence is not necessarily a causal one (Ferguson \& Hartley, 2009; Lim, Carrotte, \& Hellard, 2016). Some, such as Ferguson and Hartley (2009), have even suggested that pornography use "may actually provide a catharsis to alleviate sexual aggression" (p. 328).

The evidence on exposure to pornography featuring aggression appears more conclusive. Greater exposure to aggressive sexual interactions in pornography has been linked to more permissive attitudes toward aggression, coercion, and sexual violence toward women (Malamuth, Addison, \& Koss, 2000; Wright \& Tokunaga, 2016), as well as to a greater proclivity to commit sexual assault (Boeringer, 1994; Hald, Malamuth, \& Yuen, 2010; Milburn, Mather, \& Conrad, 2000). Foubert, Brosi, and Bannon (2011), for example, surveyed 489 US college fraternity men (aged 18-23), finding that men's use of three forms of pornography (mainstream, sadomasochistic, and rape) was associated with an increased belief in rape myths, an intent to commit rape and sexual assault in hypothetical situations, and a decreased likelihood to intervene in a hypothetical rape situation. Notably, these deleterious effects increased along with the level of violence depicted within the pornography viewed. Similarly, a longitudinal (2006-2009) US national survey demonstrated that adolescents who intentionally viewed violent pornography were almost six times more likely to report sexually aggressive behavior than those who had not (Ybarra, Mitchell, Hamburger, Diener-West, \& Leaf, 2011).

Recent review studies have suggested that even viewing nonviolent pornography may be associated with attitudes condoning violence against women (Hald et al., 2010). Meta-analyses of experimental and correlational studies have similarly shown that exposure to pornography, both with or without consent, was associated with a significant increase in the acceptance of rape myths (Allen et al., 1995; Malamuth, 2018; Yang \& Youn, 2012). Some studies have even suggested that pornography might increase the likelihood of committing sexual aggression, including the use of physical force or verbal coercion to obtain sex (Wright, Tokunaga, \& Kraus, 2016) and dating violence (Rothman \& Adhia, 2016). However, such an association with actual coercion and aggression remains doubtful (Kingston, Federoff, Firestone, Curry, \& Bradford, 2008; Richters et al., 2008; Vogels \& O’Sullivan, 2019).

Of note, neither experimental nor correlational data provide much information about subjective experiences with pornography, including the negotiation and ambivalence involved in the use of pornography. In real-life social and sexual interactions beyond the laboratory, what constitutes aggression may be far more complex than a pre-defined and narrow understanding of aggression, as it was set out in laboratory studies. We begin addressing this gap with the help of our qualitative interviews with pornography viewers, which we discuss in the final section of this chapter. 


\section{Qualifying and moderating factors in the pornography-aggression relationship}

The relationship between pornography use and aggression is further complicated by moderating factors, primarily individual characteristics such as antisocial or aggressive tendencies (see Kingston et al., 2009; Malamuth \& Hald, 2017). Psychological studies on male pornography users have reported that traits such as hostile masculinity, narcissism, and low agreeableness were associated with a greater risk for real-life sexual aggression (Hald \& Malamuth, 2015; Hald, Malamuth, \& Lange, 2013; Malamuth, 2018). Malamuth (1981), one of the first to suggest this interaction, found that exposure to nonconsenting pornography was more evocative of sexual aggression for men who already exhibited tendencies toward sexual aggression (as measured by survey responses that determined the degree of "force orientation").

Gender may be another moderating factor impacting the relationship between pornography use and aggression. Most studies on the relationship between pornography use and sexual aggression have been conducted with men. The few studies that examined women found that they had stronger negative affective reactions to pornography, were less likely to be aroused by content they considered to be degrading, and anticipated worse outcomes for viewers (Attwood, 2005; Glascock, 2005; Goodson, McCormick, \& Evans, 2001; Hald, 2007). Still, studies that examined the effects of aggressive content in pornography on women found that those who read pornographic materials that included violence were more likely to blame the victims rather than the perpetrators of the act (Davis, Norris, George, Martell, \& Heiman, 2006; Norris, Davis, George, Martell, \& Heiman, 2004). Hald and Malamuth (2015) similarly reported that higher levels of pornography consumption among women predicted attitudes supporting violence against women.

In sum, recent research suggests that the effects of pornography exposure likely depend on a large number of qualifying and moderating factors. These include gender, the type of pornography being consumed, the frequency of use, the degree of active search for aggressive content, the specific outcome in question (e.g. attitudes, behaviors, or endorsement of aggression toward women), and various individual characteristics, such as levels of agreeableness and antisocial tendencies (Malamuth \& Huppin, 2005; Miller, Hald, \& Kidd, 2018; Vega \& Malamuth, 2007). Psychologist Michael Bader therefore concluded that "porn is not harmless. But neither is it an important cause of sexual violence or misogyny. Partisans on both sides of this debate have littered their arguments with distortions, hyperbole, and cheap rhetorical tricks" (Tarrant, 2016, p. 94).

\section{Pornography's effects on adolescents and youth}

Adolescents and young adults are the heaviest consumers of pornography (Pornhub.com, 2015), with 61\% of the visitors to PornHub in 2018 aged 
18 to 34 . A US study of 433 youth, aged 12-22, found that $85 \%$ of males and $50 \%$ of females reported exposure to sexually explicit websites (BraunCourville \& Rojas, 2009). In addition, as we discussed in Chapter 2, studies have suggested that the average age of initial exposure to online pornography has been dropping in recent years, with many beginning to consume pornographic materials even before reaching their teenage years (Harrison \& Ollis, 2015; Lewis, Somers, Guy, Watchirs-Smith, \& Skinner, 2018; McIntyre \& Clark, 2015). With pornography's increasing accessibility, educators, scholars, and journalists have all expressed concerns that pornography functions as a primary source of sexual education for adolescents and youth (Morgan, 2011). These concerns intensify when considering that adolescence is a period of both social and neurological malleability (see Brown \& Wisco, 2019). Neuropsychological development patterns may explain the growing evidence for compulsive behaviors related to online pornography and cybersex among adolescents (Delmonico \& Griffin, 2008; Lam, Peng, Mai, \& Jing, 2009; van den Eijnden, Spijkerman, Vermulst, van Rooij, \& Engels, 2010).

Concerns about pornography as a tool for sex education are particularly applicable to young males, who both view it more frequently and hold more favorable views about it (Johansson \& Hammarén, 2007; Miller et al., 2018). Indeed, adolescents frequently report using pornography to learn about sex (Orenstein, 2016; Ramlagun, 2012). And although they report being able to distinguish between pornography and sexual reality, adolescents still consider pornography to be a reliable source of information (Löfgren-Mårtenson \& Månsson, 2010), both adopting and adapting sexual scripts obtained from pornography (Arnett, 2006; Furman \& Shaffer, 2003). Thus, pornography often serves as a "how-to" guide and as a means for young people to gauge social norms around sex, sexuality, and gender.

Empirical research on the connections between pornography use and sexual aggression has often concluded that pornography use among adolescent boys is associated with harmful attitudes and behaviors (Bloom \& Hagedorn, 2015; Springate \& Omar, 2013). These include viewing women solely as sexual objects (Peter \& Valkenburg, 2009), less progressive attitudes about gender roles (Brown \& L'Engle, 2009), and increased feelings of hostility toward women (Hald et al., 2013). Peter and Valkenburg (2016) reviewed 20 years of academic research on the prevalence, predictors, and implications of pornography use among adolescents, finding that pornography use was related to more sexual aggression, both in terms of perpetration and victimization. Longitudinal research has also found that the use of sexually explicit material, particularly violent pornography without consent, was associated with various forms of sexual violence among boys (Brown \& L'Engle, 2009; Ybarra \& Thompson, 2018). Among adolescent girls, studies found an association between pornography use and sexual victimization (Bekele, Van Aken, \& Dubas, 2011; Bonino, Ciairano, Rabaglietti, \& Cattelino, 2006; Romito \& Beltramini, 2015). Female adolescents and emerging adults who used pornography were also more likely to report emulating behaviors they 
have seen online (Maas \& Dewey, 2018; Maas et al., 2019; Mattebo, Tydén, Häggström-Nordin, Nilsson, \& Larsson, 2016).

While these studies show a relationship between pornography use and adolescents' sexual attitudes and behaviors, caution is again required before concluding about a causal relationship. Furthermore, the ways in which adolescents interact with and apply the material (aggressive or otherwise) they see in pornography often do not correspond with the linear effects assumed by traditional social learning theories (Steele \& Brown's, 1995). Mulholland's (2013) work, for example, shows that youth negotiate pornography in complex ways, demonstrating an ability to watch it for sexual satisfaction, while at the same time being able to parody it. Hence, studies have highlighted the critical skills that many adolescents employ when engaging with pornography (see Buckingham \& Bragg, 2004; Peter \& Valkenburg, 2016).

\section{The potential beneficial effects of pornography}

While the bulk of pornography scholarship focuses on its negative effects (for a review, see Davis \& Bauserman, 1993), some research highlights potential positive effects (see Newmahr, 2010). McKee's (2007) survey of pornography users in Australia found that 58\% of respondents perceived pornography as having had a very positive or a positive effect on their attitudes toward sexuality, while 35\% felt that it had no effect and $7 \%$ thought it had negative effects. Another Australian survey, with a large representative sample (aged 16-69 years), found that most pornography viewers stated that pornography did not affect them negatively (men $82 \%$ and women $84 \%$ ), and very few reported that they felt addicted to pornography (Rissel et al., 2017).

Pornography's positive effects may also include general sexual education for some young adults (McKee, 2007; Rissel et al., 2017; Smith, Attwood, \& Barker, 2018) and for those who might not have access to relevant sexual health information, primarily among sexual minorities (Kubicek, Beyer, Weiss, Iverson, \& Kipke, 2009). In addition, pornography may constitute a sphere in which individuals (e.g. LGBTQ+ individuals) can achieve a sense of identity and membership (Smith et al., 2018). For example, viewing online pornography may allow socially or sexually anxious or marginalized individuals to find and form virtual communities in which they can communicate or practice some sexual behaviors in a relatively safe environment (Cooper, Delmonico, \& Burg, 2000; Leiblum, 1997). There is also some qualitative evidence to suggest that pornography can function to expand users' sexual horizons, which may provide a source of personal empowerment (see Weinberg, Williams, Kleiner, \& Irizarry, 2010). The data we present in the next section from our interviews with pornography viewers further emphasizes the importance of empirical evidence on pornography's neutral or positive impacts. 


\section{Self-perceived effects: Findings from our interviews with pornography viewers}

The study of self-perceived effects of pornography use is a relatively young but growing subfield within pornography research (see Hald \& Malamuth, 2008; Rissel et al., 2017; Miller et al., 2018). Investigating claims about pornography's effects from the perspective of consumers is important, particularly given the dynamic and bidirectional nature of the media-consumer relationship discussed earlier (see Slater, 2007). Our interviews with 122 individuals who regularly watch pornographic videos online provide an additional contribution to this emerging subfield.

When we asked about their general view of the relationship between pornography viewing and real-life aggression (particularly violence against women), most of our interviewees believed that the two were related. Nearly three-quarters (74\%) of our interviewees felt that there was some relationship between pornography and violence against women, while $17 \%$ were unsure about the existence of such a relationship. Less than $10 \%$ of the interviewees believed that there was no relationship at all between the two. Interviewees talked about performers' economic constraints and systemic exploitation, as well as pornography's disproportionately negative impact on women, given men's acceptance and enactment of harmful scripts emulated from mainstream pornography.

However, despite these views, most of the interviewees argued that viewing has had a fairly minimal impact on their own sexual and daily practices. Less than $30 \%$ of our interviewees felt that pornography impacted their lives and even fewer felt that it had negatively impacted their lives. Many of the interviewees felt that they were relatively immune to the long-term impacts of aggression and violence in pornography. One commonly cited reason for this perceived lack of effect was not using pornography frequently enough to have an effect. Others felt that pornographic materials, including more aggressive materials, did not impact them because, over time, they have learned to use pornography "responsibly," developing "porn literacy" (Dawson, Gabhainn, \& MacNeela, 2019). Khalid, a 22-year-old heterosexual student from Canada pronounced this view:

You can't let [pornography] define your sex, [or] you'll never have realistic sex ... I have evolved. I was childish before, very straightforward. Now I see this more as a comparison to what I do in real life. Like, would this work, will it bother me? I think about how it affects my life.

Interviewees' perceptions regarding which acts are aggressive and/or humiliating clearly shaped their views on whether aggression in pornography has had a negative impact on their lives. Most participants who reported adopting certain aggressive acts (primarily consensual spanking, hair pulling, and light choking) into their sexual lives as a result of pornography use perceived these 
acts as acceptable or even desirable. Consequently, they believed that pornography has had a neutral or even a positive effect on their lives (see also Newmahr, 2010; Rissel et al., 2017). For example, Chloe, a 26-year-old bisexual business entrepreneur from France, credited pornography with clarifying her desires and revealing that she likes aggression in the context of BDSM: "it helped me know what I like and what limits I have, and understand that it's ok to like the BDSM things. It's not traumatizing; it's pleasurable and there is respect. It's not just violence." Other participants, such as Katrina, a 27-yearold heterosexual student from Slovenia and Oliver, a 26-year-old from Canada (heterosexual and unemployed), noted that pornography had "broadened their horizons" or encouraged them to try new things.

Still, many of the interviews contained contradictions regarding the behavioral implications of aggression in pornography. In speaking about the aggression they sought, encountered, or enjoyed in pornography, about one-quarter of the participants (both men and women) either implicitly or explicitly expressed discomfort or ambivalence about enjoying aggressive acts or scenes. James, a 22-year-old heterosexual student from the United Kingdom admitted that "[a]t the time [of watching aggressive porn] it's arousing. But after [you finish watching] you feel bad. You feel like 'OMG, what a horrible person I am.",

Others expressed similar sentiments. Floriane, a 22-year-old heterosexual student from Canada, remarked: "I feel hypocritical. I wouldn't want to be treated the way I like to see in porn." Elise, another 22-year-old heterosexual student from Canada, acknowledged that although she "enjoy[s] somewhat aggressive sex, it feels kind of wrong; it feels un-feminist." Jennifer, a 27-year-old heterosexual student from China similarly explained: "I'm not saying I don't occasionally dabble in more aggressive/rape-ier [material]. But when I do, my conscience tells me it's wrong." These views reflect a degree of cognitive dissonance, which appears to be due to both personal preferences and awareness of social norms and moral codes. We further explore these contrasting emotions in Chapter 6.

Some of the interviewees felt conflicted about aggressive pornography's impact on their own and on their partners' desires. For example, Kai, a 22-year-old male student from Canada, shared with us the conflict between viewing aggressive pornography and trying it in his own sex life, on the one hand, and his perception that it might lead to negative relational implications, on the other:

[Porn] makes me objectify women. I have tried rougher stuff I saw in porn, although usually it's the girl who wants to try it, weirdly enough. But on a deeper level, it kind of lessens the intimate connection we have. The aggressiveness of porn in general makes us less connected.

One very common way in which participants dealt with the dissonance between their awareness of societal norms regarding sexual aggression and their own arousal by it was to draw a very clear distinction between reality and 
fantasy, clearly situating pornography within the latter realm. For example, Alani, a 34-year-old heterosexual government administrator from Canada, said that she enjoyed aggression in pornography:

I like rougher contact... As long as it's fantasy it's okay. I really like the aggressive stuff... It's not like I want to be raped. I just like extreme dominance. But yeah, it's weird to be attracted to [this type of pornography].

Mindy, an 18-year-old heterosexual student from Sri Lanka, made a similar distinction: "I like watching gangbangs, but it's a fantasy; I wouldn't want it in real life."

These interviewees and others thus distinguished between their real-life desires, which mostly did not include aggression, and their fantasies and desires when consuming pornography, which involved seeking out and at times enjoying aggressive material. For many of them, the separation between the two worlds was clear. For example, Cindy, an 18-year-old bisexual retail worker from Canada, told us: "It's for my imagination. Like, I'd never act on it [aggressive material seen in porn] in real life." Similarly, Julie, a 23-year-old bisexual student from Mauritius, shared her approach to aggressive pornography: "I do like it, but you can't do it in real life." While most of the interviewees referred to the distinction between fantasy and reality, others chose to compartmentalize or ignore porn-related tastes and broader questions of morality. One prominent example for this approach came from Camila, a -27-year-old queer restaurant worker from the United States:

These days I don't think about the aggression I see in porn. I just enjoy it and feel good. I used to feel weird about it, guilty for enjoying it, but I've gotten over it. I don't care anymore... it doesn't need to be justified. It's just porn, and it's just sex; things that people are into sexually doesn't reflect how they are in other ways.

It thus appears that at least some of the interviewees, particularly women, who managed to enjoy or remain unaffected by aggression in pornography, were able to achieve this due to a degree of certainty that such acts would not or could not enter their own sexual scripts. Similar views were pronounced by some of the men we interviewed interviewees. Henry, a 20-year-old heterosexual student from Hong Kong, shared his thoughts about this issue with us:

I do think porn is more fantasy life for me. Things like threesomes or foursomes turn me on. I don't think that would actually be something I want in real life. So for me, porn is fantasy world vs. sex life overall.

Similarly, Veronique, 27-year-old from France who works in the film industry, repeatedly highlighted the unrealistic images in pornography as the reason she was unfazed and unaffected by aggressive material. For participants, 
then, there is a clear awareness of and investment in the "fantastical" element of aggression in pornography. This distinction between pornographic scripts and their own sexual life underpinned viewers' assessment regarding the relatively minor impact of pornography in general, and aggressive pornography in particular, has had on their personal lives.

Roughly $60 \%$ of the interviewees in our sample indicated a degree of familiarity with or even normalization of more aggressive material over time. However, in contrast to claims made by proponents of desensitization and content progression theses (see Hald et al., 2014; Mann, Berkowitz, Sidman, Starr, \& West, 1974), many young men expressed an increased aversion to aggressive material. For example, Landon, a 25-year-old heterosexual student from Canada, told us: "I think I'm more bothered now by aggressive stuff. Maybe earlier, I'd look for some aggressive stuff. But now I never think about watching it." Amin, a 22-year-old heterosexual student from India, similarly noted: "I'm more and more repelled. People go to new heights every day in porn, I can't stand it."

In line with the findings of Löfgren-Mårtenson and Månsson’s (2010) study of Swedish adolescents, our interviewees indicated not only a process of normalization but also of ambivalence toward pornography. Participants felt a range of negative emotions (e.g. uneasiness, disgust, shame, and guilt) about some of the aggression they had encountered, even while sometimes they enjoyed this aggression. However, like the participants in Löfgren-Mårtenson and Månsson's study, most of our interviewees felt that with time they have acquired the skills (for example, porn literacy) to deal with pornography "responsibly" and "reflexively." Danica, a 20-year-old sexually fluid student from Canada, exemplified this sentiment:

When I first started watch[ing] porn, like [at the age of] 14, I still had weird guilty feelings about sexuality that weren't resolved, so I stopped watching for a while. And then, when I became more comfortable with sexuality and exploring my sexuality, I started using [porn] in a healthy way around [the age of] 17. Now it's just natural.

\section{Conclusion}

This chapter provided an overview of the weedy field of research on the effects of pornography among various subpopulations. Although there is a growing scholarly consensus that pornography has both positive and negative effects, and that it may be only one of many factors leading some individuals to enact sexual aggression and violence, pornography's effects on users and on society remain debated. Existing research has not established an association between general pornography use and sexual aggression. It does, however, suggest a relationship between pornography that includes aggression and real-life sexual aggression. Still, scholars remain undecided regarding the 
causal nature of this relationship and the relationship is further complicated by multiple qualifying and moderating factors.

Participants in our qualitative sample held ambivalent stances toward aggression in the pornography they consumed as well as its broader and long-term impacts on their own lives. Importantly, however, more than two-thirds of them did not feel pornography had any impact on their own (sex) lives, challenging the extant literature focusing on pornography's progressively deleterious impacts among frequent users. Some of the interviewees mentioned "porn literacy" as a possible reason for this lack of effect. This explanation highlights the importance of developing such literacy, perhaps through educative programs with adolescents and emerging adults, who may be less able to make sense of and "compartmentalize" the sexual contents they watch. Our interviews also emphasized the tendency to bracket pornography's scripts and norms as "fantasy," thus reducing their impact on the real world of sexual interactions.

\section{Note}

1 Behavioral social scientists explain behavior as a function of external objects that act as stimuli, while symbolic interactionists emphasize the mediating importance of the meaning (arising from social interaction) individuals ascribe to external stimuli (Longmore, 1998).

\section{References}

Ågmo, A., Turi, A. L., Ellingsen, E., \& Kaspersen, H. (2004). Preclinical models of sexual desire: Conceptual and behavioral analyses. Pharmacology Biochemistry and Behavior, 78(3), 379-404. doi:10.1016/j.pbb.2004.04.013.

Allen, M., D’Alessio, D., \& Emmers-Sommer, T. M. (1999). Reactions of criminal sexual offenders to pornography: A meta-analytic summary. Annals of the International Communication Association, 22(1), 139-169. doi:10.1080/23808985.1999. 11678961.

Allen, M., Emmers, T., Gebhardt, L., \& Giery, M. A. (1995). Exposure to pornography and acceptance of rape myths. The Journal of Communication, 45(1), 5-26. doi:10.1111/j.1460-2466.1995.tb00711.x.

Arnett, J. (2006). Emerging adulthood: The winding road from the late teens through the twenties. New York: Oxford University Press.

Attwood, F. (2005). What do people do with porn? Qualitative research into the comsumption, use, and experience of pornography and other sexually explicit media. Sexuality and Culture, 9(2), 65-86. doi:10.1007/s12119-005-1008-7.

Bauserman, R. (1996). Sexual aggression and pornography: A review of correlation research. Basic and Applied Social Psychology, 18(4), 405-427. doi:10.1207/ s15324834basp1804_4.

Bekele, A. B., Van Aken, M. A. G., \& Dubas, J. S. (2011). Sexual violence victimization among female secondary school students in eastern Ethiopia. Violence and Victims, 26(5), 608-630. doi:10.1891/08866708.26.5.608.

Berger, R. J., Cottle, C. E., \& Searles, P. (1991). Feminism and pornography. Westport, CT: Praeger Publishers. 
Bloom, Z. D., \& Hagedorn, W. B. (2015). Male adolescents and contemporary pornography: Implications for marriage and family counselors. The Family Journal, 23(1), 82-89. doi:10.1177/1066480714555672.

Boeringer, S. B. (1994). Pornography and sexual aggression: Associations of violent and nonviolent depictions with rape and rape proclivity. Deviant Behavior, 15(3), 289-304. doi:10.1080/01639625.1994.9967974.

Bonino, S., Ciairano, S., Rabaglietti, E., \& Cattelino, E. (2006). Use of pornography and self-reported engagement in sexual violence among adolescents. European Journal of Developmental Psychology, 3(3), 265-288. doi:10.1080/174056206 00562359.

Braun-Courville, D. K., \& Rojas, M. (2009). Exposure to sexually explicit web sites and adolescent sexual attitudes and behaviors. Journal of Adolescent Health, 45(2), 156-162. doi:10.1016/j.jadohealth.2008.12.004.

Brom, M., Both, S., Laan, E., Everaerd, W., \& Spinhoven, P. (2014). The role of conditioning, learning and dopamine in sexual behavior: A narrative review of animal and human studies. Neuroscience \& Biobehavioral Reviews, 38(1), 38-59. doi:10.1016/j.neubiorev.2013.10.014.

Brown, J. A., \& Wisco, J. J. (2019). The components of the adolescent brain and its unique sensitivity to sexually explicit material. Journal of Adolescence, 72(1), 10-13. doi:10.1016/j.adolescence.2019.01.006.

Brown, J. D., \& L'Engle, K. L. (2009). X-rated: Sexual attitudes and behaviors associated with U.S. early adolescents' exposure to sexually explicit media. Communication Research, 36(1), 129-151. doi:10.1177/0093650208326465.

Brownmiller, S. (1975). Against our will. New York, NY: Simon and Schuster.

Buckingham, D., \& Bragg, S. (2004). Young people, sex and the media: The facts of life? Hampshire \& New York: Palgrave Macmillan.

Carpenter, L. M. (1998). From girls into women: Scripts for sexuality and romance in seventeen magazine, 1974-1994. Journal of Sex Research, 35(2), 158-168. doi:10.1080/00224499809551929.

Cavalier, R. (6 December 2011). "Feminism and pornography". CMU Philosophy Department. Retrieved from http://caae.phil.cmu.edu/cavalier/Forum/ pornography/ background/CMC_article.html

Chapkis, W. L. (1995). Prostitution politics and policies: An examination of the commercial sex trade. Ph.D. dissertation, University of California, Santa Cruz.

Ciclitira, K. (2004). Pornography, women and feminism: Between pleasure and politics. Sexualities, 7(3), 281-301. doi:10.1177/1363460704040143.

Cooper, A., Delmonico, D. L., \& Burg, R. (2000). Cybersex users, abusers, and compulsives: New findings and implications. In A. Cooper (Ed.), Cybersex: The dark side of the force. Sexual addiction \& compulsivity (pp. 5-29). Philadelphia, PA: Brunner-Routledge.

Courtice, E. L., \& Shaughnessy, K. (2018). The partner context of sexual minority women's and men's cybersex experiences: Implications for the traditional sexual script. Sex Roles, 78(3-4), 272-285. doi:10.1007/s11199-017-0792-5.

Davis, C. M., \& Bauserman, R. (1993). Exposure to sexually explicit materials; An attitude change perspective. Annual Review of Sex Research, 4(1), 121-209.

Davis, K. C., Norris, J., George, W. H., Martell, J., \& Heiman, J. R. (2006). Rapemyth congruent beliefs in women resulting from exposure to violent pornography: Effects of alcohol and sexual arousal. Journal of Interpersonal Violence, 21(9), 1208-1223. doi:10.1177/0886260506290428. 
Dawson, K., Gabhainn, S. N., \& MacNeela, P. (2019): Toward a model of porn literacy: Core concepts, rationales, and approaches. The Journal of Sex Research, 00(0). doi:10.1080/00224499.2018.1556238.

Delmonico, D. L., \& Griffin, E. J. (2008). Cybersex and the E-teen: What marriage and family therapists should know. Journal of Marital and Family Therapy, 34(4), 431-444. doi:10.1111/j.1752-0606.2008.00086.x.

Diamond, M. (2009). Pornography, public acceptance and sex related crime: A review. International Journal of Law and Psychiatry, 32, 304-314. doi:10.1016/j. ijlp.2009.06.004.

Diamond, M., Jozifkova, E., \& Weiss, P. (2011). Pornography and sex crimes in the Czech Republic. Archives of Sexual Behavior, 40, 1037-1050. doi:10.1007/ s10508-010-9696-y.

Dines, G. (2010). Pornland: How porn has hijacked our sexuality. Boston, MA: Beacon Press.

Dines, G., Jensen, R., \& Russo, A. (1998). Pornography: The production and consumption of inequality. New York, NY: Routledge.

Donnerstein, E. (1980). Aggressive erotica and violence against women. Journal of Personality and Social Psychology, 39(2), 269-277. doi:10.1037//0022-3514.39.2.269.

Donnerstein, E. (1984). Pornography: Its effect on violence against women. In N. M. Malamuth \& E. Donnerstein (Eds.), Pornography and sexual aggression (pp. 53-81). Orlando, FL: Academic Press.

Duggan, L., Hunter, N., \& Vance, C. (1994). False promise: Feminist antipornography legislation. In A. Jaggar (Ed.), Living with contradictions (pp. 165-170). Boulder, CO: Westview Press.

Durham, M. (1996). The taming of the shrew: Women's magazines and the regulation of desire. Journal of Communication Inquiry, 20(1), 18-31. doi:10.1177/019685999602000102.

Dworkin, A. (1981). Pornography: Men possessing women. London, UK: Women's Press.

Eaton, A. W. (2007). A sensible antiporn feminism. Ethics, 117(4), 674-715. Retrieved from http://web.mit.edu/sgrp/2008/no2/EatonSAPF.pdf

Ferguson, A. (1984). Sex war: The debate between radical and libertarian feminists. Chicago Journals, 10(1), 106-112.

Ferguson, C. J., \& Hartley, R. D. (2009). The pleasure is momentary...the expense damnable? The influence of pornography on rape and sexual assault. Aggression and Violent Behavior, 14(5), 323-329. doi:10.1016/j.avb.2009.04.008.

Foubert, J. D., Brosi, M. W., \& Bannon, R. S. (2011). Pornography viewing among fraternity men: Effects on bystander intervention, rape myth acceptance and behavioral intent to commit sexual assault. Sexual Addiction \& Compulsivity, 18(4), 212-231. doi:10.1080/10720162.2011.625552.

Furman, W., \& Shaffer, L. (2003). The role of romantic relationships in adolescent development. In P. Florsheim (Ed.), Adolescent romantic relations and sexual behavior: Theory, research, and practical implications (pp. 3-22). Mahwah, NJ: Lawrence Erlbaum Associates, Inc.

Glascock, J. (2005). Degrading content and character sex: Accounting for men and women's differential reactions to pornography. Communication Reports, 18(1-2), 43-53. doi:10.1080/08934210500084230.

Goodson, P., McCormick, D., \& Evans, A. (2001). Searching for sexually explicit materials on the Internet: An exploratory study of college students' behavior and attitudes. Archives of Sexual Behavior, 30(2), 101-118. doi:10.1023/A:1002724116437. 
Hald, G. M. (2007). Gender differences in pornography consumption among young heterosexual Danish adults. Archives of Sexual Behavior, 35(5), 577-585. doi:10.1007/ s10508-006-9064-0.

Hald, G. M., \& Malamuth, N. M. (2008). Self-perceived effects of pornography consumption. Archives of Sexual Behavior, 37(4), 614-625. doi:10.1007/ s10508-007-9212-1.

Hald, G. M., \& Malamuth, N. M. (2015). Experimental effects of exposure to pornography: The moderating effect of personality and mediating effect of sexual arousal. Archives of Sexual Behavior, 44(1), 99-109. doi:10.1007/s10508-014-0291-5.

Hald, G. M., Malamuth, N. M., \& Lange, T. (2013). Pornography and sexist attitudes among heterosexuals. The Journal of Communication, 63(4), 638-660. doi:10.1111/ jcom.12037.

Hald, G. M., Malamuth, N. M., \& Yuen, C. (2010). Pornography and attitudes supporting violence against women: Revisiting the relationship in nonexperimental studies. Aggressive Behavior, 36(1), 14-20. doi:10.1002/ab.20328.

Hald, G. M., Seaman, C., \& Linz, D. (2014). Sexuality and pornography. In D. Tolman, L. Diamond, J. Bauermeister, W. George, J. Pfaus, M. Ward (Eds.), APA handbook of sexuality and psychology (pp. 3-35). Washington, DC: American Psychological Association.

Hardy, S. (1998). The reader, the author, his woman, and her lover: Soft-core pornography and heterosexual men. London, UK: Cassell.

Harrison, L., \& Ollis, D. (2015). Young people, pleasure, and the normalization of pornography: Sexual health and well-being in a time of proliferation?. In J. Wyn \& H. Cahill (Eds.), Handbook of children and youth studies (pp. 155-167). Singapore: Springer.

Jensen, R. (2007). Getting off: Pornography and the end of masculinity. Cambridge, MA: South End Press.

Johansson, T., \& Hammarén, N. (2007). Hegemonic masculinity and pornography: Young people's attitudes toward and relations to pornography. The Journal of Men's Studies, 15(1), 57-70. doi:10.3149/jms.1501.57.

Kaminer, W. (12June 2013). When conservative senators soundlike anti-porn feminists. The Atlantic. Retrieved from https://www.theatlantic.com/sexes/archive/2013/06/ when-conservative-senators-sound-like-anti-porn-feminists/276808/

Kennedy, A., Kimmel, M., \& Llewellyn, C. (2013). The porn wars redux: What can young feminists learn from the porn wars, and what can those veterans learn from younger feminists. Revista Anglo Saxonica, 3(6), 177-200. Retrieved from https:// www.academia. edu/3356278/Beauty_Incarnate_A_Claim_for_Postmodern_ Feminist_Theology

Kim, J. L., Lynn Sorsoli, C., Collins, K., Zylbergold, B. A., Schooler, D., \& Tolman, D. L. (2007). From sex to sexuality: Exposing the heterosexual script on primetime network television. Journal of Sex Research, 44(2), 145-157. doi:10.1080/00224490701263660.

Kingston, D. A., Federoff, P., Firestone, P., Curry, S., \& Bradford, J. M. (2008). Pornography use and sexual aggression: The impact of frequency and type of pornography use on recidivism among sexual offenders. Aggressive Behavior, 34(4), 341-351. doi:10.1002/ab.20250.

Kingston, D. A., Malamuth, N. M., Fedoroff, P., \& Marshall, W. L. (2009). The importance of individual differences in pornography use: Theoretical perspectives 
and implications for treating sexual offenders. Journal of Sex Research, 46(2-3), 216-232. doi:10.1080/00224490902747701.

Kipnis, L. (1998). Bound and gagged: Pornography and the politics of fantasy in America. Durham, NC: Duke University Press.

Kubicek, K., Beyer, W. J., Weiss, G., Iverson, E., \& Kipke, M. D. (2009). In the dark: Young men's stories of sexual initiation in the absence of relevant sexual health information. Health Education and Behaviour, 37(2), 243-263. doi: 10.1177/1090198109339993.

Kutchinsky, B. (1991). Pornography and rape: Theory and practice? Evidence from crime data in four countries where pornography is easily available. International Journal of Law and Psychiatry, 14, 47-64.

Lam, L. T., Peng, Z. W., Mai, J. C., \& Jing, J. (2009). Factors associated with internet addiction among adolescents. Cyberpsychology \& Behavior, 12(5), 551-555. doi:10.1089/cpb.2009.0036.

Leiblum, S. R. (1997). Sex and the net: Clinical implications. Journal of Sex Education and Therapy, 22(1), 21-27. doi:10.1080/01614576.1997.11074167.

Lewis, L., Somers, J. M., Guy, R., Watchirs-Smith, L., \& Skinner, S. R. (2018). 'I see it everywhere': Young Australians' unintended exposure to sexual content online. Sexual Health, 15(4), 335-341. doi:10.1071/SH17132.

Lim, M. S., Carrotte, E. R., \& Hellard, M. E. (2016). The impact of pornography on gender-based violence, sexual health and well-being: What do we know?. Journal of Epidemiology \& Community Health, 70(1), 3-5. doi:10.1136/jech-2015-205453.

Lindner, K., Blosser, G., \& Cunigan, K. (2009). Visual versus auditory learning and memory recall performance on short-term versus long-term tests. Modern Psychological Studies, 15(1). Retrieved from https://scholar.utc.edu/mps/vol15/iss1/6

Linz, D., \& Malamuth, N. M. (1993). Pornography. London, UK: Sage.

Lipton, S. (2012). Trouble ahead: Pleasure, possibility and the future of queer porn. New Cinemas: Journal of Contemporary Film, 10(2-3), 197-207. doi:10.1386/ ncin.10.2-3.197_1.

Löfgren-Mårtenson, L., \& Månsson, S. A. (2010). Lust, love, and life: A qualitative study of Swedish adolescents' perceptions and experiences with pornography. Journal of Sex Research, 47(6), 568-579.

Longmore, M. A. (1998). Symbolic interactionism and the study of sexuality. The Journal of Sex Research, 35(1), 44-57. doi:10.1080/00224499809551916.

Maas, M. K., \& Dewey, S. (2018). Internet pornography use among collegiate women: Gender attitudes, body monitoring, and sexual behavior. Sage Open, $8(2)$, 1-9. doi:10.1177/2158244018786640.

Maas, M. K., Bray, B. C., \& Noll, J. G. (2019). Online sexual experiences predict subsequent sexual health and victimization outcomes among female adolescents: A latent class analysis. Journal of Youth and Adolescence, 48(5), 837-849. doi:10.1007/ s10964-019-00995-3.

Malamuth, N. M. (1981). Rape fantasies as a function of exposure to violent sexual stimuli. Archives of Sexual Behavior, 10(1), 33-47. doi:10.1007/BF01542673.

Malamuth, N. M. (Ed.). (1984). Pornography and sexual aggression (1st Ed.). Orlando, FL: Academic Press.

Malamuth, N. M. (2018). "Adding fuel to the fire"? Does exposure to non-consenting adult or to child pornography increase risk of sexual aggression? Aggression and Violent Behavior, 41, 74-89. 
Malamuth, N. M., Addison, T., \& Koss, M. (2000). Pornography and sexual aggression: Are there reliable effects and can we understand them?. Annual Review of Sex Research, 11(1), 26-91.

Malamuth, N. M., \& Check, J. V. (1980). Sexual arousal to rape and consenting depictions: The importance of the woman's arousal. Journal of Abnormal Psychology, 89(6), 763-766. doi:10.1037/0021-843X.89.6.763.

Malamuth, N. M., \& Check, J. V. (1981). The effects of mass media exposure on acceptance of violence against women: A field experiment. Journal of Research in Personality, 15(4), 436-446.

Malamuth, N. M., \& Hald, G. M. (2017). The confluence mediational model of sexual aggression. In A. R. Beech \& T. Ward (Eds.), Volume I: Theories. The wiley handbook on the theories, assessment and treatment of sexual offending (2nd ed., pp. 53-71). Oxford, UK: Wiley.

Malamuth, N. M., Hald, G. M., \& Koss, M. P. (2012). Pornography, individual differences in risk and men's acceptance of violence against women in a representative sample. Sex Roles, 66, 427-439.

Malamuth, N. M., \& Huppin, M. (2005). Pornography and teenagers: The importance of individual differences. Adolescent Medicine Clinics, 16(2), 315-326. doi:10.1016/j.admecli.2005.02.004.

Mann, J., Berkowitz, L., Sidman, J., Starr, S., \& West, S. (1974). Satiation of the transient stimulating effect of erotic films. Journal of Personal Social Psychology, 30(6), 729-735. doi:10.1037/h0037528.

Mattebo, M., Tydén, T., Häggström-Nordin, E., Nilsson, K. W., \& Larsson, M. (2016). Pornography consumption among adolescent girls in Sweden. The European Journal of Contraception \& Reproductive Health Care, 21(4), 295-302. doi:10.1080/ 13625187.2016.1186268.

McBridge, A. (2011). Lesbian history: The sex wars. University of Michigan. Retrieved from https://archive.ph/20120719152558/http://sitemaker.umich.edu/ lesbian.history/the_sex_wars

McElroy, W. (1997). XXX: A woman's right to pornography. New York, NY: St Martin's Press.

McIntyre, S., \& Clark, D. (2015). The role of technology in human trafficking. A white paper prepared for Microsoft. Retrieved from http://mtroyal.ca/cs/groups/ public/documents/pdf/ humantrafficking.pdf

McKee, A. (2007). 'Saying you've been at dad's porn book is part of growing up': Youth, pornography and education. Metro Magazine, 155, 118-122. Retrieved from https://eprints.qut.edu.au/14574/1/14574.pdf

McKee, A. (2014). The relationship between attitudes towards women, consumption of pornography, and other demographic variables in a survey of 1,023 consumers of pornography. International Journal of Sexual Health, 19(1), 31-45. doi:10.1300/ J514v19n01_05.

Mellman, W. L. (2017). Beyond the gender binary in sexual scripts?: Dating and relationships among transgender men and their non-transgender partners (Doctoral dissertation). Columbia University, New York.

Milburn, M. A., Mather, R., \& Conrad, S. D. (2000). The effects of viewing R-rated movie scenes that objectify women on perceptions of date rape. Sex Roles, 43(9-10), 645-664. doi:10.1023/A:1007152507914.

Miller, D. J., Hald, G. M., \& Kidd, G. (2018). Self-perceived effects of pornography consumption among heterosexual men. Psychology of Men \& Masculinity, 19(3), 469-476. doi:10.1037/men0000112. 
Morgan, E. M. (2011). Associations between young adults' use of sexually explicit materials and their sexual preferences, behaviors, and satisfaction. Journal of Sex Research, 48(6), 520-530. doi:10.1080/00224499.2010.543960.

Mulholland, M. (2013). Young people and pornography: Negotiating pornification (pp. 99-120). Palgrave Macmillan, New York.

Newmahr, S. (2010). Rethinking kink: Sadomasochism as serious leisure. Qualitative Sociology, 33(3), 313-331. doi:10.1007/s11133-010-9158-9.

Norris, J., Davis, K. C., George, W. H., Martell, J., \& Heiman, J. R. (2004). Victim's response and alcohol-related factors as determinants of women's responses to violent pornography. Psychology of Women Quarterly, 28(1), 59-69. doi:10.1111/j.1471-6402.2004.00123.x.

Orenstein, P. (2016). When did porn become sex ed? New York Times. Retrieved August 9, 2018 from https://www.nytimes.com/2016/03/20/opinion/sunday/ when-did-porn-become-sex-ed.html

Peter, J., \& Valkenburg, P. M. (2006). Adolescents' exposure to sexually explicit material on the Internet. Communication Research, 33(2), 178-204. doi:10.1177/0093650205285369.

Peter, J., \& Valkenburg, P. M. (2009). Adolescents' exposure to sexually explicit internet material and notions of women as sex objects: Assessing causality and underlying processes. Journal of Communication, 59(3), 407-433. doi:10.1111/j.1460-2466.2009. 01422.x.

Peter, J., \& Valkenburg, P. M. (2016). Adolescents and pornography: A review of 20 years of research. The Journal of Sex Research, 53(4-5), 509-531. doi:10.1080/ 00224499.2016 .1143441$.

Pornhub.com. (2015). Coming of age: Millennials. Pornhub. Retrieved August 9, 2018, from http://www.pornh ub.com/insights/millennials-demographics-statistics

Pornhub.com. (2018, January 9). Pornhub's 2017 year in review. Retrieved from https://www.Pornhub.com/insights/2017-year-in-review

Ramlagun, P. (2012). "Don't call me weird, but I normally watch porn"-Girls, sexuality and porn. Agenda, 26(3), 31-37. doi:10.1080/10130950.2012.716652.

Richters, J., De Visser, R. O., Rissel, C. E., Grulich, A. E., \& Smith, A. M. (2008). Demographic and psychosocial features of participants in bondage and discipline, "sadomasochism" or dominance and submission (BDSM): Data from a national survey. The Journal of Sexual Medicine, 5(7), 1660-1668. doi:10.1111/j.1743-6109.2008.00795.x.

Rissel, C., Richters, J., de Visser, R. O., McKee, A., Yeung, A., \& Caruana, T. (2017). A profile of pornography users in Australia: Findings from the second Australian study of health and relationships. Journal of Sex Research, 54(2), 227-240. doi:10.1080/00224499.2016.1191597.

Romito, P., \& Beltramini, L. (2015). Factors associated with exposure to violent or degrading pornography among high school students. The Journal of School Nursing, 31(4), 280-290. doi:10.1177/1059840514563313.

Rothman, E. F., \& Adhia, A. (2016). Adolescent pornography use and dating violence among a sample of primarily Black and Hispanic, urban-residing, underage youth. Behavioral Sciences, 6(1), 1. doi:10.3390/bs601 0001.

Rubin, G. (1984). Thinking sex: Notes for a radical theory of the politics of sexuality. In C. Vance (Ed.), Pleasure and danger: Exploring female sexuality (pp. 267-319). Boston, MA: Routledge.

Rubin, G. (1998). Social perspectives in lesbian and gay studies. New York City, NY: Routledge, 100-133. 
Seto, M. C., Maric, A., \& Barbaree, H. E. (2001). The role of pornography in the etiology of sexual aggression. Aggression and Violent Behavior, 6, 35-53.

Simon, W., \& Gagnon, J. H. (1986). Sexual scripts: Permanence and change. Archives of Sexual Behavior, 15(2), 97-120. doi:10.1007/BF01542219.

Simon, W., \& Gagnon, J. H. (2003). Sexual scripts: Origins, influences and changes. Qualitative Sociology, 26(4), 491-497. doi:10.1023/B:QUAS.0000005053.99846.e5.

Slater, M. D. (2007). Reinforcing spirals: The mutual influence of media selectivity and media effects and their impact on individual behavior and social identity. Communication Theory, 17(3), 281-303. doi:10.1111/j.1468-2885.2007.00296.x.

Smith, C. (2018). Policy, politics and porn. Sexualities, 21(8), 1351-1359. doi:10.1177/1363460718794416.

Smith, C., Attwood, F., \& Barker, M. (2018). 'I'm just curious and still exploring myself': Young people and pornography. New Media \& Society, 20(10), 3738-3759. Retrieved from http://sure.sunderland.ac.uk/id/eprint/8988

Springate, J., \& Omar, H. A. (2013). The impact of the Internet on the sexual health of adolescents: A brief review. International Journal of Child and Adolescent Health, 6(4), 469-471. Retrieved from https://uknowledge.uky.edu/pediatrics_facpub/135

Steele, J. R., \& Brown, J. D. (1995). Adolescent room culture: Studying media in the context of everyday life. Journal of Youth and Adolescence, 24, 551-576. doi:10.1007/ BF01537056.

Tarrant, S. (2016). The pornography industry: What everyone needs to know. New York, NY: Oxford University Press.

van Den Eijnden, R. J., Spijkerman, R., Vermulst, A. A., van Rooij, T. J., \& Engels, R. C. (2010). Compulsive internet use among adolescents: Bidirectional parentchild relationships. Journal of Abnormal Child Psychology, 38(1), 77-89. doi:10.1007/ s10802-009-9347-8.

Vega, V., \& Malamuth, N. M. (2007). Predicting sexual aggression: The role of pornography in the context of general and specific risk factors. Aggressive Behavior, 33, 104-117. doi:10.1002/ab.20172.

Vogels, E. A., \& O’Sullivan, L. F. (2019). The relationship among online sexually explicit material exposure to, desire for, and participation in rough sex. Archives of Sexual Behavior, 48(2), 653-665. doi:10.1007/s10508-018-1290-8.

Watson, M. A., \& Smith, R. D. (2012). Positive porn: Educational, medical, and clinical uses. American Journal of Sex Education, 7, 122-145. doi:10.1080/15546128. 2012.680861.

Weinberg, M. S., Williams, C. J., Kleiner, S., \& Irizarry, Y. (2010). Pornography, normalization, and empowerment. Archives of Sexual Behavior, 39(6), 1389-1401. doi:10.1007/s10508-009-9592-5.

Weitzer, R. (2011). Sex trafficking and the sex industry: The need for evidence-based theory and legislation. The Journal of Criminal Law \& Criminology, 101(4), 1337-1370. Retrieved from https://heinonline.org/HOL/P?h=hein.journals/jclc101\&i=1347

Whisnant, R., \& Stark, C. (Eds.). (2004). Not for sale: Feminists resisting prostitution and pornography. North Geelong, AU: Spinifex Press.

Whittier, N. (2014). Rethinking coalitions: Anti-pornography feminists, conservatives, and relationships between collaborative adversarial movements. Social Problems, 61(2), 175-193. doi:10.1525/sp.2014.12151.

Willis, E. (18 October 2005). Lust horizons. Village Voice. Retrieved from http:// www.villagevoice.com/10/18/lust-horizons/i 
Wright, P. J. (2012). Show me the data! Empirical support for the "centerfold syndrome". Psychology of Men \& Masculinity, 13(2), 180.

Wright, P. J., Miezan, E., \& Sun, C. (2018). Pornography consumption, perceptions of pornography as sexual information, and condom use. Journal of Sex \& Marital Therapy, 44(8), 800-805. doi:10.1080/0092623X.2018.1462278\.

Wright, P. J., Sun, C., Steffen, N. J., \& Tokunaga, R. S. (2015). Pornography, alcohol, and male sexual dominance. Communication Monographs, 82(2), 252-270. doi: 10.1080/03637751.2014.981558.

Wright, P. J., \& Tokunaga, R. S. (2016). Men's objectifying media consumption, objectification of women, and attitudes supportive of violence against women. Archives of Sexual Behavior, 45(4), 955-964. doi:10.1007/s10508-015-0644-8.

Wright, P. J., Tokunaga, R. S., \& Kraus, A. (2016). A meta-analysis of pornography consumption and actual acts of sexual aggression in general population studies. Journal of Communication, 66(1), 183-205. doi:10.1111/jcom.12201.

Wright, P. J., Tokunaga, R. S., Kraus, A., \& Klann, E. (2017). Pornography consumption and satisfaction: A meta-analysis. Human Communication Research, 43(3), 315-343. doi:10.1111/hcre.12108.

Yang, D. O., \& Youn, G. (2012). Effects of exposure to pornography on male aggressive behavioral tendencies. The Open Psychology Journal, 5(1), 1-10. doi: 10. 2174/1874350101205010001.

Ybarra, M. L., Mitchell, K. J., Hamburger, M., Diener-West, M., \& Leaf, P. J. (2011). $\mathrm{X}$-rated material and perpetration of sexually aggressive behavior among children and adolescents: Is there a link? Aggressive Behavior, 37(1), 1-18. doi:10.1002/ Ab.20367.

Ybarra, M. L., \& Thompson, R. E. (2018). Predicting the emergence of sexual violence in adolescence. Prevention Science, 19(4), 403-415. doi:10.1007/ s11121-017-0810-4.

Zhou, Y., \& Paul, B. (2016). Lotus blossom or dragon lady: A content analysis of "Asian women" online pornography. Sexuality \& Culture, 20(4), 1083-1100. doi:10.1007/s12119-016-9375-9. 


\section{$5 \quad$ Myths about the prevalence of aggression}

In this chapter we reexamine two common assumptions that often appear in the academic literature and in popular media, though typically without the backing of systematic empirical support. First, we reexamine the common claim that the vast majority of (popular) pornography contains aggression. Second, we evaluate the validity of claims that mainstream online pornography is getting "harder and harder," that is, that it contains a steadily growing amount of aggressive acts.

\section{Assertion I: the vast majority of mainstream pornography contains aggression}

Former estimates of the prevalence of aggression in rental and Internet pornographic videos have varied greatly, ranging from about $\%$ (McKee, 2005) to almost 90\% (Bridges, Wosnitzer, Scharrer, Sun, \& Liberman, 2010) of all videos. This wide range partly reflects different definitions of aggression, but it is also the result of examining different forms of media at different time periods and of varying methodological and sampling choices.

Despite these diverging estimates, many scholars and journalists tend to cite the higher-end estimates and claim that the (large) majority of pornography available on the Internet contains aggression, particularly aggression toward women. In academia, this view is especially popular among radical feminists such as Gail Dines (2003, 2006, 2010) and Robert Jensen (2007, 2010, 2016), who are frequently interviewed and cited by highly circulated mainstream media, pronouncing the long-held view that pornography is violence against women. Jensen, for example, believes that "pornography is what the end of the world looks like" (2010, p. 105), while Dines argues that "pornography has socialized a generation of men into watching sexual torture" (Hedges, 2015). The statement that about $90 \%$ of pornography is violent has also been widely cited in articles on pornography by leading mainstream media, such as the BBC (Brown, 2017) The Daily Telegraph (Tuohy, 2015), The Independent (Smith, 2013), The Washington Post (Halverson, 2016), The Guardian (Bindel, 2010, 2019), and The Sydney Morning Herald (McKenzie, 2011). 
Table 5.1 The prevalence of aggression in PornHub videos

\begin{tabular}{llc}
\hline & $\begin{array}{l}\text { Visible } \\
\text { aggression (\%) }\end{array}$ & $\begin{array}{l}\text { Non-consensual } \\
\text { aggression (\%) }\end{array}$ \\
\hline Most-watched videos of all time sample $(n=70)$ & 12.9 & 1.4 \\
Random PornHub sample $(n=76)$ & 36.8 & 9.2 \\
Overall sample, including various racial & 39.8 & 12.3 \\
$\quad$ categories $(n=269)$ & & \\
\hline
\end{tabular}

How accurate are these claims? How prevalent is aggression in today's mainstream free online pornography? Our analyses show that regardless of specific definitions and sampling choices, the claim that the (large) majority of free online pornography contains aggression is unsubstantiated. In Table 5.1 we present our findings for our sample of PornHub videos from the years 2008 to 2016. We examined both visible and non-consensual aggression, ${ }^{1}$ as well as various samples and sub-samples from PornHub, including a random sample of all available videos, a sample of the all-time most-watched videos, and a larger purposive sample, in which we oversampled from various racial categories.

As Table 5.1 shows, when we examine only the most-watched videos in PornHub, aggression tends to be sporadic. This sample is important, as these are the videos to which PornHub viewers are most likely to be exposed. Thus, these videos potentially have the highest degree of influence on viewers' sexual scripts and sexual education. In addition, this sampling technique is most comparable to some of the most notable and well-cited previous studies in this field (e.g. Bridges et al., 2010; Klaassen \& Peter, 2015; McKee, 2005). Only $12.9 \%$ of the 70 videos we examined contained any form of visible aggression. Most of this aggression was in the form of what one might call "milder" aggression - acts such as moderate spanking (of the bottom) and hair pulling-while "harder" acts of aggression, such as choking, forced gagging, and face hitting was visibly less common. Furthermore, we have argued in Chapter 3 for the importance of differentiating between consensual and non-consensual aggression, noting that even if consent is problematic within the context of the pornography industry, the message to viewers when an act is clearly non-consensual is more powerful and pernicious. As such, it is important to note that only $1.4 \%$ of the videos in the most-watched sample (that is, one out of the 70 videos) contained non-consensual aggression.

Table 5.1 also presents figures for a random sample of PornHub videos, retrieved using the website's "random" function $(n=76)$ and for a larger sample that also contains videos specifically sampled from PornHub subcategories that focus on racial and ethnic minorities $(n=269)$. Aggression was more prevalent in these two samples, although it still did not exceed $40 \%$ of all videos. Only $36.8 \%$ of the videos in the random sample and $39.8 \%$ of the videos in the overall sample contained any form of visible aggression. Non-consensual aggression was present in $9.2 \%$ and $12.3 \%$ of the videos, 
respectively. We therefore conclude that while aggression is certainly present in mainstream online pornography, it tends to be quite infrequent in the most-watched videos (particularly "harsher" forms of aggression) and less common than previously reported even in less-frequently-watched videos. Furthermore, non-consensual aggression is infrequent and one is not likely to encounter it often, unless specifically searching for this type of videos.

Beyond noting whether a given video included aggression or not, it is also important to assess how central aggression was to the narrative of the videos in which it appeared. We therefore also calculated the relative share of a given video in which aggression was depicted. We found that even videos that included aggressive acts were not all aggressive. In these videos, visible aggression was portrayed in about $6 \%$ of the entire duration of the video, while other acts and behaviors, including displays of affection and pleasure, almost always featured more prominently. In the large majority of the videos in which aggression was included, aggression was not the main theme but rather one among multiple components of the sexual act. In other words, the sexual script entailed by these videos is not one where aggression dominates all other acts but instead one where aggressive acts are part of the fuller repertoire of sexual encounters.

\section{Which aggressive acts are most prevalent?}

Our measure of "visible aggression" included various acts, most of which were also included in former studies on aggression in pornography (e.g. Bridges et al., 2010; Klaassen \& Peter, 2015). However, the frequency of these acts varies significantly. For example, kicking, which was signaled as one potential form of aggression by former studies, did not appear in any of the 269 videos in our sample. Other acts, such as hitting a woman's body or face, were also very infrequent. Table 5.2 shows that the most common acts categorized as aggression in our sample of most-watched videos $(n=70)$ were hair pulling and spanking, although even these were fairly infrequent, with $5.71 \%$ and $7.14 \%$ respectively. These two acts were also the most common ones in our random sample $(n=76)$, particularly spanking, with almost one-quarter of the videos in the sample including this act. When further expanding the sample to include the various racial categories $(n=269)$, two other acts emerge as also relatively common, in addition to spanking (16.36\%) - forceful vaginal penetration ("thumping"), appearing in $13.38 \%$ of the videos, and forced gagging, which appeared in $17.84 \%$ of the videos.

Table 5.2 also presents data on acts that were not typically considered aggressive in most former studies but are nevertheless considered degrading or humiliating by some authors and speakers. These include anal penetration, ejaculation in a woman's mouth, and ejaculation on her face. Of these, ejaculation in the mouth was most common, appearing in more than $30 \%$ of the videos, regardless of sampling choice. Unlike acts that were coded as aggression, ejaculation in a woman's mouth or on her face was more prominent in 
Table 5.2 The prevalence of specific aggressive/degrading acts in PornHub videos

\begin{tabular}{lccc}
\hline & $\begin{array}{l}\text { Most watched } \\
(\%)(n=70)\end{array}$ & $\begin{array}{l}\text { Random }(\%) \\
(n=76)\end{array}$ & $\begin{array}{l}\text { Overall }(\%) \\
(n=269)\end{array}$ \\
\hline Visible aggression acts & & & \\
Hair pulling & 5.71 & 7.89 & 7.43 \\
Spanking & 7.14 & 23.68 & 16.36 \\
Rough handling & 1.43 & 2.63 & 8.92 \\
Choking & 1.43 & 3.95 & 3.35 \\
Face hitting & 0.00 & 3.95 & 1.49 \\
Body hitting & 1.43 & 3.95 & 2.97 \\
Forced gagging & 4.29 & 6.58 & 17.84 \\
Forceful vaginal penetration & 2.86 & 1.32 & 13.38 \\
Forceful anal penetration & 1.43 & 1.32 & 4.09 \\
& & & \\
Acts that some consider degrading & 8.57 & 9.21 & 11.90 \\
Anal penetration & 35.71 & 31.58 & 31.60 \\
Ejaculation in mouth & 24.29 & 18.42 & 22.68 \\
Ejaculation on face & & & \\
\hline
\end{tabular}

the most-watched video sample than in the random sample or the overall sample. This suggests that these acts are the ones that have been most normalized and that they have been adopted as a fairly standard script in mainstream pornography. Below, we explore how viewers perceive these acts and respond to them.

\section{What acts do viewers perceive as aggression?}

While our definition of visible aggression follows previous research in this field, one question that is not asked enough in these studies is how viewers themselves interpret these acts. Do viewers indeed view all of these acts as aggressive? Our interviews with consumers of online pornography therefore provide further context for the figures presented above. When we asked our interviewees what acts they considered to be aggressive in the pornography videos that they watch, the large majority of them cited consent (both verbal and visual) as the primary and most important signal for whether or not they viewed an act as aggressive. Of note, viewers brought up consent even without us prompting them about this issue. For example, Danica, a 20-year-old Canadian student, told us:

I think generally, anything is okay, as long as it's consensual. Unless I see the person is in pain. Then I cringe and think: "that's gotta be uncomfortable; that's not acceptable." In some cases, I get skeptical. They probably don't have much of a choice in it. Or the director could be exploiting the actors. [But generally, ] anything aggressive is ok as long as they're ok with it. It can be ambiguous sometimes and they might look like they're 
not totally comfortable with it. But it's not my position to say what's ok. Everyone has their own kinks. I tend to not put value judgments on it, I just follow my own preferences... all of them except gagging are ok. It depends on the extent. [Even] gagging can be self-induced, in which case it's not [aggression], but with the illusion of aggression it could be. It depends on context and extent... In terms of humiliation, it depends on [the] dynamic. It can be powerful for the person receiving, if you're in a more assertive position and say you want it. It's not an act in itself; it's who is navigating the situation.

Julie, a 23-year-old student from Mauritius, shared similar sentiments:

I think it depends. If it's [the act] consensual, it's okay. But if it's not, then it's aggression... [An act is] acceptable as long as you see it's not too much, or there is any visible pain. If it looks like torture, it's not okay. As long as it's not too much though, acts like spanking, hitting, and gagging are acceptable.

Most of our male interviewees also shared these views and emphasized consent as a central feature when thinking about aggression in pornography and making sense of it. Henry, a 20-year-old student from Hong Kong, told us:

As long as it's consensual, then no, it's not aggressive... Hair pulling, for example, depends on the extent. If it's sudden and done without knowledge of consent, then yes, it's aggressive. Gagging and spanking, if it's consensual, it's not aggressive, but spanking can also be super aggressive and continuous, and you can see that it hurts. That bothers me. The occasional spank isn't aggressive though. Spanking in moderation can be arousing. Gagging as well, it just can't go too far. Some spanking and hair pulling is okay.

Even viewers who found aggression to be arousing, almost unanimously stressed the importance of consent. For example, Liang, a 24-year-old accounting agent from Canada, said that "as long as there is consent, aggression is okay. Consent and wanting are two different things too. But ideally, both want it." These statements about the importance of consent in interpreting an act as "aggressive" or "acceptable" (interviewees did not always make a clear distinction between these two concepts) are important in further contextualizing our findings regarding the prevalence of aggression. As shown in Table 5.1 , we found that about $9 \%$ of the randomly sampled videos and $1.4 \%$ of the most-watched videos on PornHub contained non-consensual aggression.

These findings suggest that since most viewers do not actively seek aggressive content (see later in this chapter), they would probably judge $90.8 \%-$ $98.6 \%$ of the videos that they are most likely to watch (the exact rate depends on individual viewing habits and preferences) as not actually including 
aggression. Alternatively, viewers may perceive these videos as containing mild aggression, albeit one that is acceptable and, for many, even arousing. These figures are far lower than many previous estimates regarding the prevalence of aggression in popular online pornography. They serve to dispel the myth that the vast majority of free popular pornography portrays aggression against women or that many individuals actively seek out materials that they perceive to be aggressive.

\section{Assertion II: mainstream online pornography is getting "harder and harder"}

Another common contention among scholars and journalists writing about pornography is that while the industry may not have traditionally included large amounts of aggression, over the last decade or two, aggression has been growing steadily. According to this line of argumentation, the industry continuously pushes the boundaries of sexual conventions, becoming "harder and harder" with every passing year (Fradd, 2017; Picker \& Sun, 2008; Sun, Wosnitzer, Bridges, Scharrer, \& Liberman, 2010). Contents that were previously considered marginal and esoteric, such as rough, aggressive, and demeaning acts, gradually make their way into mainstream videos and eventually become the new norm. Some pornography scholars and pundits have argued that with time, viewers - the majority of whom are men-gradually become desensitized to such materials. Many viewers begin to actively seek aggressive and demeaning contents once the thrill and excitement previously achieved by traditional videos diminishes, in ways that are similar to drug addicts who consume higher doses once they are unable to reach the same high as with the previous dose (Doidge, 2007; Hilton \& Clark, 2011; Paul, 2010).

Some critiques of pornography have further suggested that male viewers prefer aggression and humiliation of women not merely because they present a novelty, but rather because they celebrate the tension and thrill derived from sexualizing gender inequalities in the form of men's dominance and women's submission (Dines, 2006, 2010; Dines, Jensen, \& Russo, 1998). Anti-porn critics have additionally charged that the producers, creators, and distributers of pornographic materials are more than happy to comply with the growing demand for materials that depict aggression against women and/or acts that demean and humiliate women (Picker \& Sun, 2008). These key industry personnel, who are almost all men, remain oblivious, or outright deny, the possible harms of such materials, catering to, and helping to create, public demand, all in a cynical pursuit of greater profits (Dines, 2010; Hedges, 2015; Jensen, 2007; Paul, 2005; Picker \& Sun, 2008). Consequently, and in the absence of any effective regulation, mainstream pornography steadily adopts harder content, with scenes depicting aggression and humiliation of female performers increasingly becoming the norm (Bridges et al., 2010).

Consistent with such claims, the final segment of the influential 2008 documentary, The Price of Pleasure (Picker \& Sun, 2008), was titled "Harder and 
Harder." It projected a grim future for the porn industry, one in which boundaries are consistently pushed and aggression and violence increasingly become part of mainstream pornographic videos. These predictions rely primarily on the views of renowned feminist anti-pornography scholars and investigative journalists, such as Gail Dines (2010), Karen Boyle (2010), Robert Jensen (2007, 2016), Pamela Paul (2005), and Chris Hedges (Hedges, 2015). But the assertion that pornographic video content is becoming increasingly aggressive seems nearly axiomatic, echoed in many of the current scholarly works in this field (e.g. Boyle, 2010; DeKeseredy, 2015; DeKeseredy \& Hall-Sanchez, 2017; Foubert, 2016; Fradd, 2017; Tyler, 2010; Whisnant, 2010).

It is therefore not surprising that the view that pornographic content is becoming increasingly aggressive is also very prevalent in the popular media. Articles in leading media outlets such as the BBC (Brown, 2017) The Daily Telegraph (Tuohy, 2015), The Independent (Smith, 2013), The Washington Post (Halverson, 2016), The Guardian (Bindel, 2010, 2019), and The Sydney Morning Herald (McKenzie, 2011), all talk about how pornography has been changing for the worse. These articles advance the position that pornography continues to move in the direction of rougher, harder, and more violent materials, with producers and audiences alike always looking for something more extreme and more shocking.

Some writers have also suggested possible reasons for this presumed tendency toward increasing aggression in pornographic videos. Psychiatrist Norman Doidge (2007) suggested that the very meaning of "hardcore" porn has changed over the years. "Softcore" contents today are what hardcore used to be in the past, while "hardcore" videos are increasingly dominated by themes that infuse sex with hatred and humiliation. The reason for this, Doidge argued, is that the more our society becomes sexually saturated, the more porn makers pump out harder and harder materials, trying to remain on the cutting edge. New York Times writer Judith Shulevitz (2016) has similarly argued that the Internet has made porn weirder and weirder because the flooding of the market by amateur sex tapes has cut into producers' profits. Consequently, the only way for producers to stay in business was by coming up with more extreme scenarios that satisfy the most outré desires. Shulevitz reported that she spoke with communication experts who maintained that in recent years, fetishes that one could only find on the "dark net" have become easily accessible through popular porn tube sites such as PornHub. They further argued that degrading and aggressive videos have moved into more prominent locations within these sites, making them more likely to be viewed by wider audiences.

Despite this wide agreement over the direction of aggression in pornography, none of the authors mentioned above has provided systematic empirical support for this contention. Indeed, to the best of our knowledge, such claims have never been supported by empirical evidence from large-scale systematic analyses of pornographic content. Still, the assumption that pornography is becoming increasingly aggressive has rarely been challenged and appears to have gained wide consensus in scholarly writings, journalistic reports, and consequently, as we show below, among the wider public. 


\section{Viewers' perceptions about the "harder and harder" argument}

The sense that mainstream pornography is gradually becoming aggressive was also evident in our interviews with online pornography viewers. ${ }^{2}$ When we asked interviewees whether they felt that pornography was becoming more aggressive with time, many of them responded affirmatively. However, most did not seem to consult their own experience with pornography when reflecting on this matter. Instead, they appeared to be reciting what they viewed as conventional wisdom about the reasons for this "harder and harder" trend. As such, some of them were rehearsing arguments about the perceived need of pornography websites to "up their game" in order to stay in business. For example, Ray, a 22-year-old entrepreneur from Canada, said: "There's so much choice out there, [so] companies need to differentiate themselves." Raphael, a 26-year-old sales coordinator from France, agreed: "Because there is too much porn, there is an excess. Producers want to be more unique. So yes, more hardcore." Donna, a 19-year-old student from Canada, summarized this sentiment: "There's so much done to make it more shocking and dramatic and draw attention [in order] to fight for clicks."

Other viewers also appeared to be influenced by media messages, reciting arguments about addiction and habituation. For example, William, a 25-year-old food attendant from Zimbabwe, believed that pornography is getting more aggressive "because people are trying new stuff." Mindy, an 18-year-old student from Sri Lanka, more specifically referenced arguments about habituation: "Yeah I think the barrier for pleasure is higher. People need more to get off. Porn tries to go above and beyond previous barriers." Similarly, Suzanne, a 19-year-old student from France, argued that "people grow numb to what they see. [So pornographic materials] need to go crazier [in order for viewers] to get aroused." Some interviewees echoed scholarly arguments that the presumably growing rates of aggression in pornography result from a backlash by (straight) men who perceive their social power as diminishing. Julian, a 20-year-old queer man from Canada, who works at a call center, reflected on what he saw as an increase in aggression over time: "There's something coming out, especially [in response to the] Me Too movement etc. So people are trying to take back male dominance and be able to do whatever. White hetero men are a lot of [the] audience."

It should be noted that not all of the interviewees saw the presumed increase in aggressive content as a negative development. Some believed that it reflects changes in social norms and in definitions of deviant sexuality. For example, Ray (cited above) believed that "BDSM isn't really taboo anymore. It's not really considered sexually deviant. Forty percent of [the] people do some kind of BDSM in their own lives." Similarly, Christopher, a 21-yearold student from Canada, argued that "with the way everything is moving in 2019, everything nowadays, especially with Pride, you see different types of sexual aspects that people like." James, a 22-year-old (unemployed) from the UK, agreed: "The definition of what's tame versus kinky is changing 
because everyone is exposed to this stuff." Ayami, a 22-year-old student from Japan, sees things in a similar way: "It's a lot more acceptable now to watch it [aggressive acts]. And there's more acceptance for all types of fetishes and scenes now, and people are more adventurous." Tristan, a 25-year-old medical student from Canada summarized this common sentiment:

Yeah for sure [pornography is getting more and more aggressive]. I think sex in general, overt behaviors, are becoming less taboo... And I think everything is becoming more and more acceptable on [the] Internet. So I think it's less restraining for porn and sexuality in general.

\section{So, is porn really becoming more aggressive?}

To answer this question, we examined videos uploaded to PornHub over the last decade. ${ }^{3}$ Almost all of the videos in our overall sample (262 of 269) were uploaded to PornHub between 2008 and 2016, with 25 to 36 videos uploaded each year within this nine-year period. In Figure 5.1, we show the percentage of videos including one of our three measures of aggression in each year between 2008 and 2016. The graph begins in 2008 because only seven videos in our sample were uploaded to PornHub prior to this year. Examining the trends in Figure 5.1 reveals no evidence for a temporal increase in aggression. None of our three measures of aggression (visible aggression, non-consensual aggression, and aggressive video titles) shows an increase over time, and in fact some of them actually show a downward trend.

First, depictions of visible aggression fluctuate but show no steady upward or downward trend. Videos depicting visible aggression mostly range from around $30 \%$ to $50 \%$ of all videos yearly (the trend for this measure was not statistically significant). Importantly though, the average duration of visible aggression in videos shows a substantial declining trend $(p<0.05)$. In 2008, nearly $13 \%$ of the average video portrayed visible aggression. By 2016, this

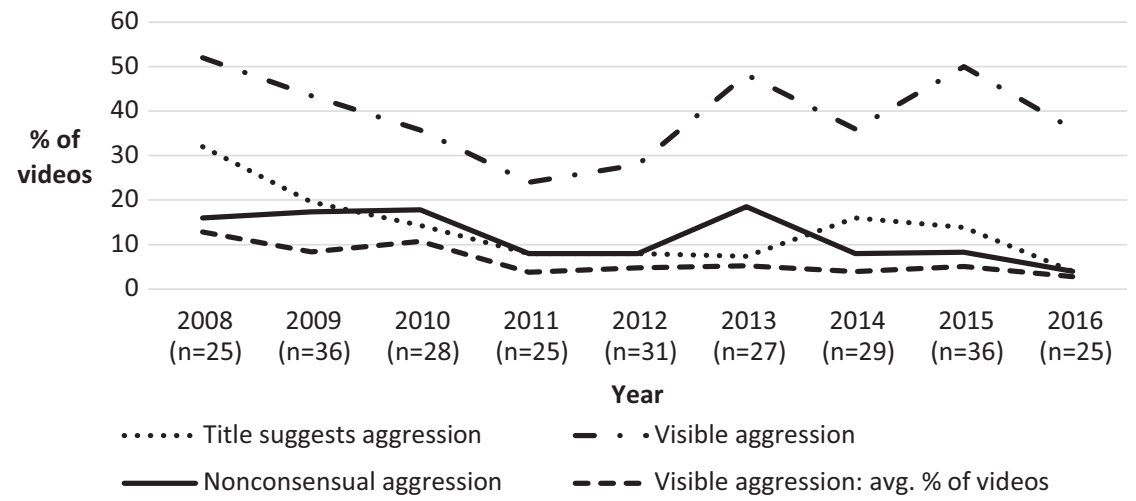

Figure 5.1 Temporal tendencies in aggression ( $n=262$ videos). 
figure dropped to less than 3\%. This finding is important, as it stresses the fact that even videos that contain aggressive acts are not all about aggression. In fact, many of them also portray female pleasure-mostly not in response to aggression-courtship, tenderness, and affection. Similarly, the rate of videos with non-consensual aggression slightly declined over time, from about $20 \%$ of all videos in 2009 and 2010 to roughly 10\% over the last three years, although this trend was not statistically significant. Finally, the prevalence of videos with titles that suggest aggression dropped significantly $(p<0.05)$ from nearly $40 \%$ of all videos in 2008 to about 5\% in 2016.

In addition to the overall time trends in aggression, which we report in Figure 5.1, we also examined time trends in each of the specific measures of aggression noted in our methodological chapter (e.g. biting, pulling hair, hitting of the body, choking, forced gagging, spanking, and forceful penetration). We found no significant trend for any of these practices. In Figure 5.2, we present four of the more common practices that appear in videos, three that are frequently coded as aggression (spanking, hair pulling, and forced gagging) and one sometimes considered to be humiliating (ejaculation on face). As noted above, none of the four practices shows a consistent statistically significant upward trend. The only practice that might be somewhat on the rise according to the figure is spanking. However, this may simply be a temporal fad and it is important to note again that most of the interviewees in our sample do not actually consider spanking to be a form of aggression.

\section{Conclusion}

In this chapter we examined two common myths about pornography: (1) that the vast majority of (popular) pornography contains aggression and (2) that online mainstream pornography is getting "harder and harder" with time. Our analyses of the content of popular videos from PornHub and of our

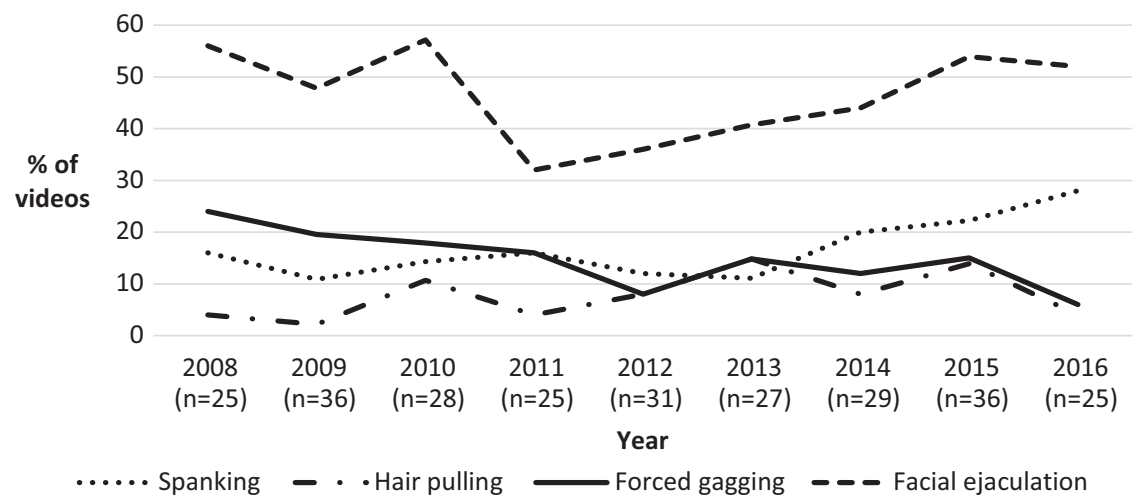

Figure 5.2 Temporal tendencies in specific aggressive acts ( $n=262$ videos). 
in-depth interviews with men and women who regularly watch online pornography provide little evidence for both of these assertions.

We found no support for the claim that most of mainstream, freely available online pornography is aggressive. First, visible aggression was present in less than $40 \%$ of the videos in a random sample from PornHub and in less than $13 \%$ of the 70 most-watched videos on this website. Second, even in videos that included aggression, aggressive acts accounted for only about $6 \%$ of the entire duration of the video and were mostly overshadowed by depictions of affection, passion, and even compassion. Third, most of these aggressive acts were ones that the viewers we interviewed actually considered to be non-aggressive-mostly due to perceived consent-or, at most, they included mild acts of aggression, such as moderate spanking and hair pulling. Finally, non-consensual acts - the only acts that most of the viewers we interviewed actually defined as aggression-were very infrequent. They appeared in about $9 \%$ of the randomly sampled videos and $1.4 \%$ of the mostwatched videos in our sample.

The second assertion, according to which pornography is getting "harder and harder," was also not supported by our data and analyses. We found no evidence for an uptake in the number of videos depicting visual aggression uploaded to PornHub over the last decade. Furthermore, we found a significant decrease in the average length of scenes that included such visibly aggressive acts, as well as an overall downward trend in the number of videos containing non-consensual aggression and in the number of video titles that suggested aggression. More specific acts, such as hair pulling, forced gagging, and facial ejaculation, also did not show an upward trend. Together, these findings suggest either a status quo or perhaps even a mild decrease in the prevalence of aggression over the last decade. Our findings do not support the common notion that many pornography users seek more "cutting edge" and aggressive materials in order to reach sexual satisfaction.

\section{Notes}

1 As we describe in greater detail in the methodological chapter (Chapter 3), our definition for "visible aggression" focuses on the acts themselves and on the apparent intent to cause harm, pain, or discomfort, while "nonconsensual aggression" considers lack of consent as key for defining an act as aggressive or violent.

2 See Chapter 3 for a detailed description of our sample of interviewees.

3 In Chapter 3, we describe in detail our research design, sampling strategy, and sample.

\section{References}

Bindel, J. (2010). The truth about the porn industry. Retrieved from https://www. theguardian.com/lifeandstyle/2010/jul/02/gail-dines-pornography 
Bindel, J. (2019). How to talk to your kids about porn (before the pornographers do). Retrieved from https://www.theguardian.com/commentisfree/2018/oct/25/ how-to-talk-to-your-kids-about-porn-pornography

Boyle, K. (2010). Introduction: everyday pornography. In K. Boyle (Ed.), Everyday pornography (pp. 1-14). New York, NY: Routledge.

Bridges, A. J., Wosnitzer, R., Scharrer, E., Sun, C., \& Liberman, R. (2010). Aggression and sexual behavior in best-selling pornography videos: A content analysis update. Violence Against Women, 16(10), 1065-1085.

Brown, J. (2017, September 26, 2017). Is porn harmful? The evidence, the myths and the unknowns. Retrieved from http://www.bbc.com/future/ story/20170926-is-porn-harmful-the-evidence-the-myths-and-the-unknowns

DeKeseredy, W. (2015). Critical criminological understandings of adult pornography and woman abuse: New progressive directions in research and theory. International Journal of Crime Justice and Social Democracy, 4(4), 4-21.

DeKeseredy, W., \& Hall-Sanchez, A. (2017). Adult pornography and violence against women in the heartland: Results from a rural southeast Ohio study. Violence Against Women, 23(7), 830-849.

Dines, G. (2003). From fantasy to reality: Unmasking the pornography industry. In R. Morgan (Ed.), Sisterhood is forever: The women's anthology for a new millennium (pp. 306-314). New York, NY: Washington Square Press.

Dines, G. (2006). The white man's burden: Gonzo pornography and the construction of black masculinity. Tale Journal of Law and Feminism, 283, 283-297.

Dines, G. (2010). Pornland: How porn has hijacked our sexuality. Boston, MA: Beacon Press.

Dines, G., Jensen, R., \& Russo, A. (1998). Pornography: The production and consumption of inequality. New York, NY: Routledge.

Doidge, N. (2007). The brain that changes itself: Stories of personal Triumph from the frontiers of brain science. London, UK: Penguin Books.

Foubert, J. (2016). How pornography harms. Bloomington, IN: LifeRichPublishing.

Fradd, M. (2017). The porn myth: Exposing the reality behind the fantasy of pornography. San Francisco, CA: Ignatius Press.

Halverson, H. (2016). The anti-porn movement is growing. The public is just catching up. Retrieved from https://www.washingtonpost.com/news/in-theory/ $\mathrm{wp} / 2016 / 05 / 27 /$ the-anti-porn-movement-is-growing-the-public-is-justcatching-up/?tid=a_inl\&utm_term $=.5$ b6363ce9792

Hedges, C. (2015). Pornography is what the end of the world looks like'. Retrieved from https://www.truthdig.com/articles/pornography-is-what-the-endof-the-world-looks-like/

Hilton, D., \& Clark, W. (2011). Pornography addiction: A neuroscience perspective. Surgical Neurology International, 2, 19-23.

Jensen, R. (2007). Getting off: Pornography and the end of masculinity. Cambridge, MA: South and Press.

Jensen, R. (2010). Pornography is what the end of the world looks like. In K. Boyle (Ed.), Everyday pornography (pp. 105-113). New York, NY: Routledge.

Jensen, R. (2016). Pornographic values: Hierarchy and hubris. Sexualization, Media, E Society, January-March, 2, 1-5.

Klaassen, M., \& Peter, J. (2015). Gender (in)equality in Internet pornography: A content analysis of popular pornographic Internet videos. Journal of Sex Research, 52(7), 721-735. 
McKee, A. (2005). The objectification of women in mainstream pornographic videos in Australia. Journal of Sex Research, 42(4), 277-290.

McKenzie, S. (2011, March 3, 2011). Why the new 'porn norm' is hurting women. Retrieved from http://www.smh.com.au/federal-politics/why-the-new-pornnorm-is-hurting-women-20110302-1be54.html

Paul, P. (2005). Pornified: How pornography is damaging our lives, our relationships, and our families. New York, NY: Henry Holt.

Paul, P. (2010). From pornography to porno to porn: How porn became the norm. In J. Stonder \& D. Hughes (Eds.), The social costs of pornography: A collection of papers (pp. 3-20). Princeton, NJ: Witherspoon Institute.

Picker, M., \& Sun, C. (Writers). (2008). The price of pleasure: Pornography, sexuality $\mathcal{E}$ relationships. Northampton, MA: Media Education Foundation.

Shulevitz, J. (2016). It's O.K., liberal parents, you can freak out about porn. Retrieved from https://www.nytimes.com/2016/07/17/opinion/sunday/its-okliberal-parents-you-can-freak-out-about-porn.html

Smith, J. (2013). Porn has changed - For the worse. Even men have noticed. Retrieved from http://www.independent.co.uk/voices/comment/porn-has-changed-forthe-worse-even-men-have-noticed-8846457.html

Sun, C., Wosnitzer, R., Bridges, A. J., Scharrer, E., \& Liberman, R. (2010). Harder and harder: The content of popular pornographic movies. In M. Antoinette Paludi \& F. Denmark (Eds.), Victims of sexual assault and abuse: Incidence and psychological dimensions (pp. 335-361). Westport, CT: Praeger.

Tuohy, W. (2015). Rougher, harder, violent: How porn is wrapping the male mind. Retrieved from http://www.dailytelegraph.com.au/rendezview/rougherharder-violent-how-porn-is-warping-the-male-mind/news-story/504698ce318f $551052847 \mathrm{f} 13 \mathrm{ac} 678 \mathrm{fd} 1$

Tyler, M. (2010). 'Now, that's pornography': Violence and domination in adult video news. In K. Boyle (Ed.), Everyday pornography (pp. 50-62). New York, NY: Routledge.

Whisnant, R. (2010). From Jekyll to Hyde: The grooming of male pornography consumers. In K. Boyle (Ed.), Everyday pornography (pp. 114-133). New York, NY: Routledge. 


\section{Myths about the popularity of aggression}

The "harder and harder" premise, which we began to explore in Chapter 5, in fact enfolds two related, yet separate, assertions. The first of these assertions is a temporal one, regarding the content of popular pornography on the Internet, which presumably contains higher levels of aggression with each passing year. As we showed in the previous chapter, we found no empirical support for this claim. The second assertion is closely related to the first. It suggests that the assumed escalation in aggressive content is fueled by an ever-increasing consumer demand. In this view, viewers of Internet pornography have come to prefer videos containing aggressive acts to those that do not contain such acts. That is, viewers are not only exposed to greater degrees of aggression; rather, they actively seek this content and prefer it to non-aggressive content.

The claim that aggression is popular among pornography viewersparticularly among male viewers - is quite prevalent in the anti-pornography literature. But how valid is this claim? Do (most) pornography viewers really seek and enjoy aggressive content? And is it also the case that men are disproportionally the audience for such content? In this chapter we explore the validity of these claims using both quantitative behavioral measures of popularity-looking at the number of views and at viewers' ratings for videos including aggression - and qualitative interviews with both men and women who frequently watch pornographic videos online.

\section{Scholarly claims about the popularity of aggression}

The assumption that men prefer aggressive and demeaning content largely relies on the increasingly popular, although contested, premise that pornography viewing often becomes an addiction since it affects the brain in a way that is similar to that of most drugs (Allen, Kannis-Dymand, \& Katsikitis, 2017; Brand, Snagowski, Laier, \& Maderwald, 2016; ,Doidge 2007; Foubert, 2016, 2017; Hilton, 2013; Hilton \& Clark, 2011; Love, Laier, Brand, Hatch, \& Hajela, 2015; Phillips, Hajela, \& Hilton, 2015). According to this view, much like any other addiction, frequent pornography use is characterized by habituation and a growing demand for more extreme substances in order to 
achieve the same rewards. Psychiatrist Norman Doidge (2007), one of the notable proponents of this approach, suggested that in individuals who compulsively and chronically watched Internet pornography, dopamine is continuously released into the reward system, which in turn simulates neuroplastic changes that reinforce the experience. With time, users develop tolerance, where previously established brain maps for "natural" sexuality can no longer compare with the newly developed maps generated by the compulsive watching of Internet pornography, pushing individuals to more explicit and graphic Internet pornography in order to maintain the higher level of excitement.

While this addiction model has been criticized by other scientists (Ley, Prause, \& Finn, 2014; Prause, Janssen, Georgiadis, Finn, \& Pfaus, 2017; van Rooij \& Prause, 2014), it remains common in clinical practice and highly popular in scholarly and media debates about pornography. Paul (2005, 2010), for example, interviewed male pornography consumers, concluding that once viewers increase the quantity of porn consumption, they then want more quality, meaning more action, more intensity, and more extreme situations. Viewers learn to ignore or navigate around unwanted imagery and gradually find previously arousing materials to be less interesting, leading them to seek more extreme and shocking materials, which often include degradation and aggression. Similarly, Whisnant (2010), reviewing online postings by individuals who discussed porn, concluded that the "contemporary pornography industry is a wasteland of lost and damaged humanity" (p. 117). She argued that hostile and humiliating acts against women are gradually becoming the rule rather than the exception, perhaps because many men actually prefer and enjoy such content, as reflected in their online comments.

Dines $(2006,2010)$ took another step in this direction, invoking claims by other radical feminists that pornography is pleasurable for men precisely because it sexualizes inequality between women and men (Brownmiller, 1975; Dworkin, 1989; Dworkin \& MacKinnon, 1988). Dines argued that since this is the case, pornography viewers-who are largely men-do not merely learn to ignore aggressive content but rather actively seek for and prefer this content. The more degraded and abused the woman is, the greater the sexual tension, thrill, and pleasure derived by male viewers. Following this logic, one may expect videos that include scenes of aggression and humiliation to be even more popular than videos that do not include such scenes. This may be particularly true for videos where women are forced into submission and clearly perform acts against their will, as this submission reinforces gender inequalities and power relations that favor men.

\section{Do viewers of mainstream pornography really prefer aggressive content?}

Despite their great popularity and traction, the contentions of scholars and journalists regarding viewers' preference for aggressive pornography have been typically based on a relatively small number of interviews and on 
anecdotal evidence. To the best of our knowledge, no previous study has systematically assessed viewers' preferences as reflected by their response to specific aggressive and non-aggressive videos and their pronounced preferences when watching such videos. In order to begin addressing this contention about the popularity of aggression in pornographic videos, we first adopt a behavioral approach, examining the content of videos available online and viewers' preferences and ratings for these videos. We therefore coded and analyzed two distinct quantitative measures of popularity: the number of views that a given video has received and its rating by viewers (measured as the relative ratio of "likes" that the videos has received).

\section{Aggression and number of views}

We first examine the number of views that a given video has received. This approach assumes that Internet users "vote with their fingers," and videos that match the preferences and fantasies of wider audiences are more likely to be watched and re-watched. Here, a comparison between our sample of "most watched videos" $(n=70)$ and our random sample of videos $(n=76)$ is useful. While the former received at least three million views each, with a mean of 23.5 million views per video, the latter received significantly fewer views, less than one million each, with a mean of 60,000 views per video.

Figure 6.1 presents our comparison between the two samples of videos for both displays of aggression and displays of pleasure. The findings presented in this figure do not support the common claims about the popularity of aggression. The figure shows that while only $12.9 \%$ of the videos in the most-viewed sample contained visible aggression and only 1.4\% (one video) included non-consensual aggression, in the random sample of less-watched videos, visible aggression was present in $36.8 \%$ of the videos and non-consensual aggression in $9.2 \%$ of them. Verbal aggression was also significantly

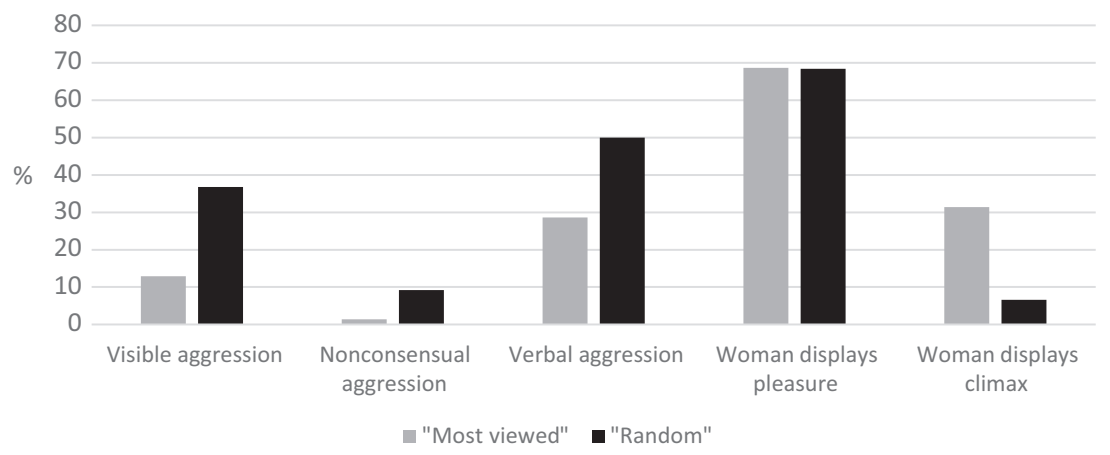

Figure 6.1 Aggression and pleasure displays in the most frequently watched ( $n=70$ ) vs. less frequently watched $(n=76)$ videos. 
more prevalent in the random sample, with half of the videos including verbal aggression (e.g. name-calling), compared with less than $30 \%$ of the videos in the most-viewed sample. As a complementary analysis, we also performed a negative binomial regression analysis, with the number of views as the dependent variable. We found that both visible aggression $(p<0.05)$ and non-consensual aggression $(p<0.01)$ were significant predictors for a video receiving fewer views.

Non-consensual aggression was particularly unpopular with viewers. This fact is consistent with McKee's $(2005,2015)$ contention that judgments about consent are at the heart of viewers' treatment of sexually explicit materials. Indeed, we found that acts such as ejaculation in mouth, anal penetration, and spanking — which some might find reprehensible or degrading-generally did not have an effect on viewers' popularity rankings (the percent of "likes"). Conversely, acts that were more clearly unpleasant/painful for female performers, such as forced gagging or forceful anal penetration, were ranked as less popular.

Furthermore, while the literature mostly focuses on displays of aggression, it is also important to examine displays of pleasure by women. One of the common claims by anti-pornography scholars and pundits is that pornography is focused on men's pleasure and neglects women's pleasure. As such, it is interesting to note the high rate of videos where women in fact display pleasure and even more to note the difference in displays of a female climax between the most frequently watched videos and the random sample. While more than $30 \%$ of the videos in the sample of most frequently watched videos included portrayals of female orgasm, this was the case for only $6.6 \%$ of the videos in the random sample. These findings suggest that viewers tend to prefer videos where women display a climax, while disfavoring those videos where women appear to be in pain.

\section{Aggression and video rating}

The results we present above offer some interesting insights into viewers' preferences. However, one possible shortcoming of "number of views" as a measure of popularity is that certain videos may receive more views not because most viewers prefer these videos, but rather because the viewers find the title or thumbnail leading to the video attractive, while these often do not clearly indicate whether the video includes aggression. Alternately, the website itself may choose to promote certain materials and make access to them easier, prompting higher viewership. Hence, the number of views may not always reflect viewers' actual preferences or opinions about the videos or the aggressive scenes within them. For example, if PornHub decides to put videos containing less aggression on its homepage, these videos may receive more views simply because many viewers see them first and click on them rather than trying to search for other content. 


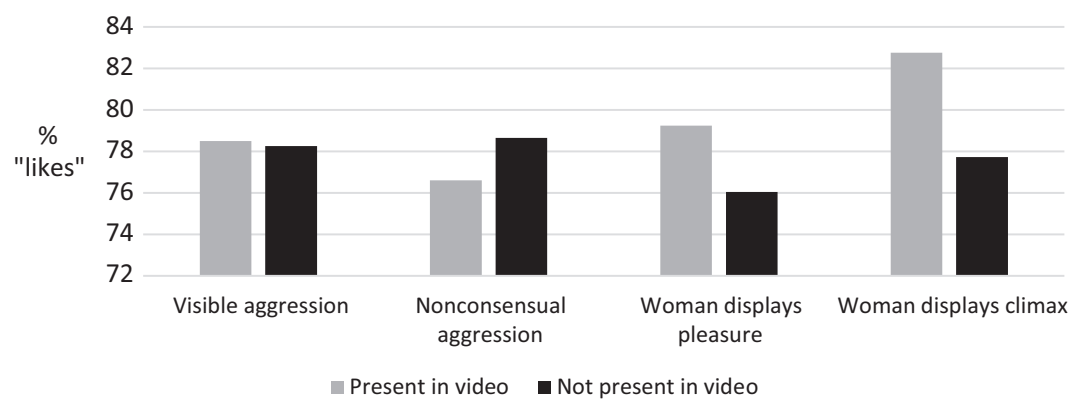

Figure 6.2 Aggression and pleasure responses as predictors of viewers' ratings of pornographic videos.

We therefore also examined an additional (alternative) and arguably more accurate measure of viewer's preferences_ video rating. PornHub allows viewers to choose whether they "liked" (green thumb up) or "disliked" (red thumb down) each of the videos on the website. For every video in our sample we coded the percent of positive ("like") votes out of the overall number of votes. The range for this measure in our sample was between $45 \%$ and $89 \%$ likes.

Using this measure, we compared the average percentage of "likes" in videos where aggression was present with the average percentage in videos where no aggression was displayed. Figure 6.2 presents the results of this comparison. The figure shows no difference in viewers' preferences between videos that displayed visible aggression and those that did not. It does, however, show a difference for non-consensual aggression, with videos portraying such aggression receiving about $2 \%$ fewer likes than videos that do not portray this type of aggression (76.6\% vs. 78.7\% respectively). While this difference is not very large, an ordinary least squares (OLS) regression analysis with popularity measured as a continuous variable showed that it is statistically significant $(p<0.05)$.

As with the number of views, we also examined the relationship between portrayals of aggression and the popularity of videos that display women's pleasure. And once again, we found a relationship that stands in contrast to statements in the literature that viewers enjoy women's suffering and pain while not caring about their pleasure. Both videos that display women's pleasure $(79.2 \%$ likes) and those displaying women's climax (82.8\% likes) were more likely to be popular than videos that did not include such pleasure displays (76.0\% likes and $77.7 \%$ likes respectively). Our OLS regression analyses further reveal that these differences were significant in both cases $(p<0.01)$. Overall, then, our results suggest that the majority of viewers who choose to share their preferences favor videos that do not include aggression and where women, rather than just men, appear to enjoy the sexual act and reach a climax. 


\section{What do viewers actually say about their preferences?}

The results we report above from our analysis of the content of videos and the responses of viewers to this content are important. They rely on the behavioral preferences of a very large number of viewers who either "voted with their fingers" by choosing to watch a certain video or chose to anonymously rate a video, which likely allowed them to freely express their actual preferences. However, these measures also suffer from a few inherent shortcomings. First, we have no way of separating the aggression or pleasure displayed in videos from other elements in the video that might have affected both the number of views and viewers' choice to award a video with a "like/dislike." We controlled for a few of these possible confounders in our regression analysis, including the performers' age and race, whether this was an amateur video or not, the year in which the video was uploaded to the website, the video's duration, and the number of views (when examining of the ratings that viewers give to videos as the dependent variable). Still, other factors may play a role in determining viewers' preferences, such as the perceived attractiveness of performers, the scenario depicted in the video, or its title (all of which do not necessarily suggest the presence or lack of aggression).

Another shortcoming of the measures we discuss above is that we lacked data on viewers' personal characteristics, such as gender, age, and nationality. In particular, gender is an important factor to consider here. The literature we reviewed above about the presumed rise of aggression in pornography and viewers' preferences for aggressive content often assumes that the large majority of viewers are men and that it is men, not women, who show a preference for such aggressive materials, thus pushing the industry to produce more of them. However, as we have noted earlier in the book (see Chapter 2 ), women actually make up a substantial portion of the viewers of freely available online pornography. It is therefore possible that, at least to a degree, the tendencies we report above are driven by female viewers, who prefer to watch less aggression and more depictions of women's pleasure, while male viewers still show a preference for aggressive acts.

Our interviews with both men and women who regularly watch online pornography are therefore instrumental in providing further insights on viewers' preferences. We asked interviewees various questions about their viewing habits and preferences related to aggression in pornography. Most of the interviewees were open about these preferences and were willing to share them with us without discernable reservations, perhaps due to the sense of anonymity offered by our phone interviews. Most of them encountered aggression in pornography. However, as indicated in Chapter 4 , their interpretation of aggressive acts often diverged from common definitions of aggression in the pornography literature, as almost all of them stressed the importance of consent (or rather lack of) in judging an act as aggressive. 


\section{Men's preferences regarding aggression}

We begin with our interviews with men, as many of the studies and journalistic reports we cite above claim that men are the primary drivers of the supposed increase in aggressive materials. Some scholars even claim that this is because many-perhaps even most-men prefer aggressive pornography to non-aggressive pornography (Dines, 2003, 2006, 2010). Our interviews provided little support for such assertions. First, more than $80 \%$ of the interviewees stated that they would not like to see more aggression in pornography. Even more strikingly, this pronounced desire was more common among men $(93.3 \%$ of the men we interviewed said they would prefer not to see more aggression) than among women (only about two-thirds of women expressed this preference). For example, Shawn, a 34-year-old Canadian student, told us that aggression was "a turn off. I don't know why it's there... I'd like to see less of it. Even if it's an act, it's a turn off." Pierre, a 29-year-old personal assistant from France, expressed similar feelings and reactions when encountering aggressive acts:

I move on. I close the video and find a good one... People don't respect each other; I don't see how people can go that low. I don't understand why you'd like it... I just feel like, yeah, I am ashamed of what society makes people do.

Lucas, a 29-year-old from France, who works in restoration, thought that " $[t]$ he porn industry should take a stand and maybe not ban everything but soften and select content that filters through."

Some interviewees even made conscious attempts to avoid aggressive pornography, aware of the message that watching such videos sends to producers. For example, Preethi, a 22-year-old student from India, told us that she has been "actively searching for non-aggressive porn, which might help propagate the idea that aggression isn't what everyone wants." Similarly, 28-year-old Arshan, also from India, noted that he consciously avoids aggressive pornography:

I find anything involving [acts such as] slapping, gagging, etc. aggressive. I just skip the content and never revert back to it. When I encounter aggressive porn, I just skip it ASAP, and also almost never visit that site [again].

In fact, the large majority of interviewees who said that they enjoyed a degree of aggression in pornography preferred to see the same amount or fewer videos containing aggression. Craig, a 25-year-old production coordinator from the United States, told us: "I would like to see less aggressive acts. I don't need to see it all over; I can search for it if needed." Similarly, Kai, a 22-year-old Chinese student, who said that he found acts such as mild spanking, hair pulling, and 
gagging arousing, said: "I would like to see less aggression; it's better. I'd like to see fewer gangbangs. That's really fucked up, it gets rough." Khalid, a 22-year-old student from Canada, also said that while he does not look for aggressive videos, he does encounter them and found it arousing when both parties were clearly enjoying the acts. However, he also said that if he could design his ideal video, "it would include intense and intimate, and passionate scenes but no violence or aggression." James, a 22-year-old from the United Kingdom, who is currently unemployed, also said that while he enjoyed some BDSM videos, his ideal video would not include these acts: "I don't think so. For me, it's demeaning... If they're not super into it, nah, I'll pass."

Most of our male interviewees $(60.0 \%$ of them) reported that they did not enjoy watching any aggressive acts in pornography, even when these acts were seemingly consensual. Most of them also expressed discomfort with being exposed to aggressive content. Furthermore, only $16.7 \%$ of the men we interviewed said that they enjoyed displays of harder aggression, ones that go beyond light spanking or hair pulling, and only two men $(3.3 \%$ of all men in the sample) said that they enjoyed non-consensual aggression. Ramy, a 25-year-old student from India, explained why he did not enjoy aggression in pornography: "[S]ex is a passion. [It is] about love, feelings, not pain or aggression." Omar, a 24-year-old science student, who grew up in both Syria and Saudi Arabia, found aggressive acts uncomfortable:

I avoid physical aggression. If it pops up, it's a turn off... I will skip video. If it's only one scene, I might skip scene. But if it's too much, I'll change the video... I saw one where there was punching in stomach, I hated it, I remember it. It's unhealthy, not something that turns me on. And it wasn't role-playing, it seemed real... I feel that it's something I don't want to do, I cringe and I don't like it, I wouldn't want to be a part of it. I just skip them.

Interestingly, however, when we asked what their ideal video would look like if they could control its content, two of our male interviewees said that they would include some aggression in it, despite their personal preference for non-aggressive content. Alex, a 24-year-old student from Russia, said: "I don't really care for them [aggressive acts]. But I guess you gotta have a bit of them, because it's the market." Similarly, Pierre, a 29-year-old personal assistant from France, said:

If I could design my "ideal" video, maybe it would have some aggression, just because it sells. If it were for me, no aggression would be there... [But] I'm okay with a small amount of aggression. It's easy to skip. Everyone has to be happy.

Barry, a 22-year-old student from Singapore, wondered whether he was doing something wrong or having the wrong sexual preferences following 
his exposure to aggressive pornographic scenes: "[It made] me question if I'm not being aggressive enough sometimes. Or if there's a general preference for aggression. I wonder if people like it and whether they are pretending or not." Much like Barry, Zach, a 24-year-old student from Canada, also said that he disliked aggressive acts but questioned whether these acts are there because other viewers enjoy watching them:

I'm not an aggressive person, I don't want to hit someone... It's not natural for me... [So when I watch aggressive acts in pornographic videos] I'm thinking: "No, I don't want to see this." But after, I think about how bad it is. I wonder how much of the aggression out there is cause people actually like it. Or people just see it all the time [and have become used to it].

Alex, Pierre, Barry, and Zach seem to believe that while they themselves do not enjoy aggressive content and would prefer not to watch it, many-or perhaps even most - pornography viewers have a preference for such content, as shown by the presence of aggressive acts in many of the videos that they have watched. They are therefore willing to make concessions and include some minor acts of aggression in their "ideal" video, just to "make everyone happy," noting that it is easy to skip such content.

However, our interviews suggest that viewers' assumptions about the pervasiveness of preferences for aggressive content (and certainly for "harder" or non-consensual aggression) are misguided. Such misconceptions may therefore lead to what social psychologists have named the Abilene Paradox (Harvey, 1974), where a group of people decide on a course of action that does not align with their personal preference because each member mistakenly believes that their own preference is counter to that of the group and therefore does not raise objections. In the current scenario, viewers may support the inclusion of some aggressive sexual acts simply because they believe such acts are normative and most — or at least many_viewers desire them.

While the majority of our male interviewees did not find aggression in pornography arousing and preferred to see fewer portrayals of aggression in mainstream free online websites, such preferences were not universal. A substantial minority among the men that we interviewed (40\%) did state that they enjoyed at least some aggression, though consensual and mostly light (that is, including acts such as light spanking and hair pulling). A small minority among our male interviewees (6\%) also expressed a desire to see more aggression in videos. Ishan, a 24-year-old PhD student from India, told us that he enjoyed some aggressive acts, particularly when it appeared that the female performers were enjoying them:

Sometimes girls want to be slapped; the pain can arouse them. If she wants it, give it to her. There are girls who want it. But I'm not sure if it's an act, or [if] they need to get paid... As long as her expression shows 
that she wants it, it's okay. Sexuality is very complicated. There's a thin line between pain and pleasure.

Nathan, a 40-year-old administrative coordinator from Canada, was more elaborate about his preferences:

[My ideal video] would include forced fellatio, slapping, dick slapping, anal, and just screaming, both in pain and in loud moaning (it would be ambiguous). I would like to see more of these... [But it] depends on how I feel. Sometimes I feel more like violent stuff; like bondage. Sometimes it's not exactly what I want. Sometimes I just want someone to be submissive, and sometimes bondage isn't that... [I enjoy] slapping on face, when someone screams a lot, when it goes fast, dick spanking/slapping. Spanking is too much, I don't like it. Hair pulling - it hurts for nothing. If it hurts without being sexually pleasing it has no interest... Sometimes it's too much, so I skip. Otherwise, I get into it. I have to admit though that I feel guilty at the end... I have also seen aggression between two men. I'd say that my main interest in these types of videos is that there is aggression in them, so I'll search specifically for aggression in gay videos. I would use keywords [like] "punishment."

Liang, a 22-year-old student, who emigrated as a child from China to Canada, expressed some similar preferences:

I would just search for categories and keywords, "BDSM," or "hairpulling," or "rough" as keywords, "bondage"... Choking, spanking, hair pulling, gagging, facials, I enjoy them. Also, I guess I enjoy holding arms back, holding someone down. I look for these and when I keep watching I am aroused. I avoid anything to do with urine/fecal matter; anything where it looks like the female is in actual pain. That ruins it for me... It's very important. I like to see female enjoying herself; ideally both parties enjoying themselves. I prefer videos that show the woman reaching an orgasm... If the dominant party gets [the] sub [the submissive party] to say something, like getting them to say "thank you," that's arousing; it's a show of dominance... I assume and hope I am applying consent correctly. I do my best to see everyone enjoy themselves, and that they can stop anytime. I have caveat of female enjoying herself. If the girl isn't enjoying it, I won't watch.

Of note, these interviewees, as well as other men we interviewed, were attracted to the exploration of the relationship between pleasure and pain in sex. All expressed interest in pornographic videos that included variations of bondage, discipline, sadism, and masochism (BDSM). And for all of them, it was important to see that the female performer was enjoying the acts. None 
of them professed to being interested in the humiliation of women for the sake of it and they all stressed that the explicit or assumed consent of the female performers was an important issue for them.

Nevertheless, these interviewees do provide some support for the view that at least some viewers have become desensitized to presentations of aggression in pornography with time and with their repeated exposure to such aggression. For some, this may also lead to seeking additional aggression or more severe forms of aggression. Liang, for example, told us:

I think I became more interested in more things over time-like the interest in BDSM. It's been a four-year thing, so as I've gotten exposure to it, I find it more arousing... Now I look for these materials more.

Similarly, Thao, a 27-year-old master's student from Vietnam, described his reaction when watching aggression:

I've become accustomed to aggressive materials over time and now I look for them more, to satisfy my curiosity. If I get bored, I try to find something more kinky or aggressive. I would go with different titles, like "bondage" or "BDSM." At first, I felt excited but also a little bit empathetic to [the] girl. But then, the more I watched it, the more I felt it's quite normal. I wasn't sure if [the] girl liked it, but then I thought they do.

Thao demonstrates here the cognitive work that some viewers go through when watching aggression in pornography. While most viewers first feel at least somewhat uncomfortable with the aggression, some of them consequently convince themselves that it is normal and suspend their disbelief, assuming that if performers are willing to do these acts, they may also like them. One possible explanation for this may be the existence of a cognitive dissonance (Festinger, 1957), where viewers are repeatedly exposed to aggressive acts that they first find reprehensible or morally wrong yet continue to watch and at times even find them arousing. Reducing moral judgment and changing their perception about the nature of the act ("performers must enjoy them") helps alleviate the dissonance and avoid unwanted and uncomfortable emotions (Grubbs \& Perry, 2019; Perry, 2017).

These accounts reveal the complex and nuanced nature of men's reaction to and occasional attraction to aggression in pornography. They negate simplistic accounts determining that many men wish to see women suffer or humiliated. Again, it is important to stress that such preferences for displays of aggression were a minority. Most of the men we interviewed clearly stated that they disliked aggression in pornography, did not search for it, and tried to avoid aggressive acts when they encountered them. 


\section{Do women who watch pornography seek aggression?}

Enjoying and seeking aggression was by no means limited to men. In fact, a higher ratio of women (65.6\%) than men (40.0\%) in our sample expressed interest in aggressive acts and found them arousing. Women were also more likely than were men to report enjoying harder forms of aggression ${ }^{1}(39.3 \%$ of the women compared with $16.7 \%$ of the men) and to seek aggression in videos $(35.0 \%$ of the women compared with $21.1 \%$ of men). For example, Bianca, a 21-year-old student from Canada, shared her preferences when watching pornography: "I like seeing spanking and hair pulling; gagging too... I also like facials [and] getting tied up; also when someone is being held down... I'd like to see more women choking guys or pulling guys' hair." Chloe, a 26-year-old entrepreneur and business owner from France, told us:

It really depends on the context. Facials can be something looked for. I find spanking, spitting, choking, and facials all okay and arousing. It depends on the context. But I don't think forced deep throat is okay at all. I find it horrible. When there's blood, it's also too much... But I would like to see more aggressive acts. My favorite website has become too soft and erotic. I would like to see more spanking, choking, and slapping.

Similarly, when we asked about her exposure to acts of aggression in pornography, Stephanie, a 41-year-old university administrator from Canada, told us:

I like it; I love it! Not necessarily rape but I like submission. For me, aggression is exciting and very arousing. I look for facials, hair pulling, spanking, gagging, all of these acts. I enjoy watching submissive/dominating materials: rape scenarios, aggressive thrusting, when women are pinned down. I find it liberating... I'm aggressive; I like to be dominated. It's a way to let go... I like to be held forcibly in positions. I like when [the] man is aggressive and dominating and when the woman has to perform stuff.

It is important to note that Stephanie seeks BDSM material but is careful to delineate the actions she feels are acceptable and unacceptable. Although aggression is clearly an integral part of BDSM, these videos also often show "after care" and commitment to women's pleasure. Alani, a 34-year-old government administrator of West Indian descent, who grew up in Canada, shared a similar penchant for aggressive pornography, while also stressing the importance of being dominated and sexual partners who take control of the situation:

I search for forced [pornography]... Gangbang; rape... I look for more submissive stuff, more like dominant males, not in whips ways, more 
in forceful ways. I don't like it when [the] woman is more dominant, that's not my thing... It's not like I want to be raped. I just like extreme dominance... I like the forcefulness of any acts; someone taking control of another... I watch and am aroused, unless it's too much, or it feels too real... [If I could design my "ideal" video] it would include forced choking, or using someone as [an] object, even if in real life I don't like that.

These quotes clarify that while a substantial number of women among our interviewees seek and enjoy aggression, the large majority of them clearly emphasize the aspects of being dominated (or dominating others) and letting others take control of the sexual interaction. Aggression for them signals passion and the lack of inhibitions and is thus both liberating and exciting. It often exists as a fantasy, which can be enjoyed precisely because it is limited to videos and does not permeate real-life sexual interactions and intimate relationships more generally.

\section{Conclusion}

In this chapter we examined common assertions that pornography viewers, particularly male viewers, prefer and actively seek out aggressive content. Our analyses of viewers' behavioral responses to popular videos in PornHub, as well as our in-depth interviews with men and women who regularly watch online pornography, provide little evidence for these assertions. In terms of behavioral measurements, videos containing aggression (in particular non-consensual aggression) were significantly less likely to be viewed than videos that did not contain aggression. Videos with more views were also more likely to include displays of women's pleasure and orgasm. In addition, videos that portrayed non-consensual aggression were less likely to receive favorable ratings from viewers, while those portraying women's pleasure and ecstasy were more likely to received favorable ratings.

These tendencies received additional support in our interviews with viewers who frequently watch online pornography. The majority (about 80\%) of both women and men who watch pornography online did not seek aggressive content. Most of them said that they did not wish to be regularly exposed to such content and many of them would have liked to see fewer aggressive acts in the videos available to them on their favorite pornography website. Moreover, these tendencies were even more pronounced among men than among women. As much as $93 \%$ of the men we interviewed stated that they would like to see less aggression in pornography, compared with about twothirds of the women we interviewed. Men were also more likely to report not enjoying any sort of aggression in videos (60\% of the men) and in particular not enjoying harder aggression (83\% of the men) and non-consensual aggression (almost all of the men). In contrast, women were more likely to find at least some forms of aggression in pornography arousing (nearly two-thirds of women), including harder forms of aggression (almost $40 \%$ of the women). 
One should be cautious not to overgeneralize these findings. In particular, we should consider the issue of interviewees' self-selection into the study. Such self-selection may mean that our interviewees are not entirely representative of the larger population of pornography viewers and it could also be that this self-selection operates differently for men and for women. We should therefore be careful not to generalize these findings to all men or to all women. In particular, we should not conclude that fantasizing about aggression and enjoying visual representations of aggression entails a desire to experience such acts in one's own sex life. Indeed, both men and women who sometimes found aggression in pornography arousing often emphasized that they would not want to experience such acts in their own sex lives.

Still, the results we present here are revealing, especially when combining the quantitative analysis of online videos ratings with the qualitative accounts coming from our interviews. The former analysis provides a concrete behavioral measurement and offer greater representativeness of pornography viewers, while the latter accounts allow for a deeper understanding of the differences between men and women and the ways in which viewers interact with this online content. Taken together, these findings stand in contrast with long-held views that most men enjoy watching aggression against women (DeKeseredy \& Corsianos, 2015; Dines, 2010; Dworkin \& MacKinnon, 1988; Russell, 1988), that they seek more of it with time (Doidge, 2007; Fradd, 2017; Hilton \& Clark, 2011; Paul, 2010), and that nearly all women are deterred by such images. We further discuss the implications of these findings in the concluding chapter.

\section{Note}

1 Defined as choking, forced gagging; kicking, hitting of the face or body parts, rough handling, and sadomasochism.

\section{References}

Allen, A., Kannis-Dymand, L., \& Katsikitis, M. (2017). Problematic internet pornography use: The role of craving, desire thinking, and metacognition. Addictive Behaviors, 70, 65-71.

Brand, M., Snagowski, J., Laier, C., \& Maderwald, S. (2016). Ventral striatum activity when watching preferred pornographic pictures is correlated with symptoms of internet pornography addiction. NeuroImage, 129(1), 224-232.

Brownmiller, S. (1975). Against our will. New York, NY: Simon and Schuster.

DeKeseredy, W., \& Corsianos, M. (2015). Violence against women in pornography. New York, NY: Routledge.

Dines, G. (2003). King Kong and the white woman: Hustler magazine and the demonization of black masculinity. In G. Dines \& J. M. Humez (Eds.), Gender, race, and class in media, (2nd Ed., pp. 451-468). Thousand Oaks, CA: Sage.

Dines, G. (2006). The white man's burden: Gonzo pornography and the construction of black masculinity. Tale Journal of Law and Feminism, 283, 283-297. 
Dines, G. (2010). Pornland: How porn has hijacked our sexuality. Boston, MA: Beacon Press.

Doidge, N. (2007). The brain that changes itself: Stories of personal Triumph from the frontiers of brain science. London, UK: Penguin Books.

Dworkin, A. (1989). Pornography: Men possessing women. New York, NY: Dutton.

Dworkin, A., \& MacKinnon, C. (1988). Pornography and civil rights: A new day for women's equality. Minneapolis, MN: Organizing Against Pornography.

Festinger, L. (1957). A theory of cognitive dissonance. Stanford, CA: Stanford University Press.

Foubert, J. (2016). How pornography harms. Bloomington, IN: LifeRichPublishing.

Foubert, J. (2017). The public health harms of pornography: The brain, erectile dysfunction, and sexual violence. Dignity, 2(3), 1-7.

Fradd, M. (2017). The porn myth: Exposing the reality behind the fantasy of pornography. San Francisco, CA: Ignatius Press.

Grubbs, J., \& Perry, S. (2019). Moral incongruence and pornography use: A critical review and integration. The Journal of Sex Research, 56(1), 29-37. doi: 10.1080/00224499.2018.1427204.

Harvey, J. (1974). The Abilene paradox: The management of agreement. Organizational Dynamics, 3, 63-80.

Hilton, D. (2013). Pornography addiction - A supranormal stimulus considered in the context of neuroplasticity. Socioaffective Neuroscience \& Psychology, 3(1), 20767.

Hilton, D., \& Clark, W. (2011). Pornography addiction: A neuroscience perspective. Surgical Neurology International, 2, 19-23.

Ley, D., Prause, N., \& Finn, P. (2014). The emperor has no clothes: A review of the 'pornography addiction' model. Current Sexual Health Reports, 6(2), 94-105.

Love, T., Laier, C., Brand, M., Hatch, L., \& Hajela, R. (2015). Neuroscience of internet pornography addiction: A review and update. Behavioral Sciences, 5(3), 388-433.

McKee, A. (2005). The objectification of women in mainstream pornographic videos in Australia. Journal of Sex Research, 42(4), 277-290.

McKee, A. (2015). Methodological issues in defining aggression for content analyses of sexually explicit material. Archives of Sexual Behavior, 44, 81-87.

Paul, P. (2005). Pornified: How pornography is damaging our lives, our relationships, and our families. New York, NY: Henry Holt.

Paul, P. (2010). From pornography to porno to porn: How porn became the norm. In J. Stonder \& D. Hughes (Eds.), The social costs of pornography: A collection of papers (pp. 3-20). Princeton, NJ: Witherspoon Institute.

Perry, S. (2017). Pornography use and depressive symptoms: Examining the role of moral incongruence. Society and Mental Health, Advance Online Publication. doi:10.1177/2156869317728373.

Phillips, B., Hajela, R., \& Hilton, D. (2015). Sex addiction as a disease: Evidence for assessment, diagnosis, and response to critics. The Journal of Treatment \& Prevention, 22(2), 167-192.

Prause, N., Janssen, E., Georgiadis, J., Finn, P., \& Pfaus, J. (2017). Data do not support sex as addictive. The Lancet Psychiatry, 4(12), 899.

Russell, D. (1988). Pornography and rape: A causal model. Political Psychology, 9(1), 41-73. van Rooij, A., \& Prause, N. (2014). A critical review of "Internet addiction" criteria with suggestions for the future. Journal of Behavioral Addictions, 3(4), 203-213.

Whisnant, R. (2010). From Jekyll to Hyde: The grooming of male pornography consumers. In K. Boyle (Ed.), Everyday pornography (pp. 114-133). New York, NY: Routledge. 


\title{
7 Sexual orientation myths
}

\author{
Is aggression less common in \\ same-sex pornography?
}

As seen in the previous two chapters, anti-pornography scholars have often claimed that pornography is a site of violence by men against women. Some further claim that this is not a coincidence. Rather, aggression by men against women and women's humiliation in pornographic videos are specifically designed to reinforce patriarchal norms and women's subordination (Dines, Jensen, \& Russo, 1998, Jensen, 2007; Whisnant \& Stark, 2004) by dictating two opposing gender and sexual roles: woman-as-submissive and man-as-dominant (Courtice \& Shaughnessy, 2018; Mellman, 2017). According to these writers and many others (e.g. Bridges, Wosnitzer, Scharrer, Sun, \& Liberman, 2010; Brownmiller, 1975; Foubert, 2016; Fradd, 2017; Hedges, 2015), pornography is inherently linked to misogyny as well as to condoning or enacting violence and sexual aggression against women.

But if one subscribes to these arguments, what would this entail regarding aggression in pornography featuring same-sex partners? Should we expect aggression in these videos to be less frequent given the absence of malefemale power dynamics and the ideology of women's submission by men? Or are these merely replicas of the power dynamics in heterosexual pornography? What are the differences in aggressive scripts between "gay," "lesbian," and "heterosexual" pornographic videos? And how do viewers make sense of such differences?

Most former studies of mainstream online pornography have focused on "heterosexual" (hereafter " $\mathrm{m} / \mathrm{f}$ ") pornography. Pornography featuring same-sex sexual interactions has received comparably little scholarly attention (Burke, 2016; Weitzer 2009). Despite a brief period of scholarly interest during the so-called feminist sex wars (see Dworkin, 1987; Rubin, 1993), both "authentic" and "ersatz" (i.e. simulated) "lesbian" (hereafter, "f/f") pornography have since been largely neglected (Cohen \& Byers, 2014; Jackson \& Gilbertson, 2009; Morrison \& Tallack, 2005). This paucity of empirical work is somewhat surprising given the great international popularity of "lesbian" scenes among male and female consumers alike (Pornhub, 2017, 2019).

Previous studies on same-sex pornography have mostly focused on "gay" (hereafter " $\mathrm{m} / \mathrm{m}$ ") pornography, particularly its use by and effects on men who have sex with men (MSM). Similar to the literature on $\mathrm{m} / \mathrm{f}$ pornography, 
the literature on online $\mathrm{m} / \mathrm{m}$ pornography can be loosely divided between "porn-positive" and "porn-negative" approaches (Bishop, 2015). The former have been adopted by studies employing a polymorphous or gay-affirmative framework, such as those that highlight the role of pornography in increasing MSM's comfort and familiarity with same-sex attractions, practices, and identity (Currin et al., 2017; Maddison, 2017) or in entering gay culture and community (Kubicek, Carpineto, McDavitt, Weiss, \& Kipke, 2011). The latter approach suggests that pornography can have a negative effect on the health of MSM, encouraging risky sexual activities (Kendall, 2004; Schrimshaw, Antebi-Gruszka, \& Downing, 2016) or increasing sexually compulsive behaviors (Nelson et al., 2014; Stein, Silvera, Hagerty, \& Marmor, 2012).

However, to our knowledge, no previous study has systematically compared the contents of $\mathrm{m} / \mathrm{f}$ and same-sex mainstream pornography. To address this gap, we analyze here data from a purposively retrieved sample of 210 videos found on PornHub. This sample includes $70 \mathrm{~m} / \mathrm{f}$ videos from the site's "most-viewed videos of all time" category (at times referred to as the "heterosexual" subsample); $70 \mathrm{~m} / \mathrm{m}$ videos from the site's "gay" category, and $70 \mathrm{f} / \mathrm{f}$ videos from the site's "lesbian" category. We compare the contents of these three subgenres, paying attention to both visible and non-consensual aggression as well as to affection and pleasure displays. We also use varying definitions of aggression, differentiating between all visible displays of aggression and aggression that is visibly non-consensual.

\section{Previous research on aggression in male/male $(\mathrm{m} / \mathrm{m})$ pornography}

As noted above, the small body of research studying aggression in the subgenre of same-sex pornography overwhelmingly focuses on male/male interactions (Brennan, 2016; Kendall, 2004, 2006; Young, 2017). Some authors espousing a harms-based paradigm call for paying attention to the overlap between $\mathrm{m} / \mathrm{f}$ and $\mathrm{m} / \mathrm{m}$ pornography, articulating a gendered power differential in which the penetrative partner dominates the receptive partner (Fung, 1991; Kendall, 1999, 2004; Mahawatte, 2004; Ortiz, 1994; Young, 2017).

Notably, these previous studies have all relied on anecdotal evidence or theoretical arguments. None of them have systematically analyzed the content of free, online $\mathrm{m} / \mathrm{m}$ pornography, nor have they focused on aggression. Still, "gay-affirmative" frameworks of $\mathrm{m} / \mathrm{m}$ pornography tend to present it in somewhat utopic terms, arguing that it can serve as an educational tool for same-sex desire (Dyer, 1994; Escoffier, 2003; Fejes \& Petrich, 1993) or safer sex practices (Levine, 1998; Watney, 1997). In addition, according to anti-pornography scholars, media representations of (hetero)sexuality through pornography are a site of violence by men against women, assisting in enforcing patriarchal norms and women's subordination through depictions of aggression or humiliation (Dines et al., 1998, Jensen, 2007; MacKinnon, 1988/2018; Whisnant \& Stark, 2004). Accordingly, we might expect 
pornography featuring interactions between two men to exhibit a relatively lower degree of aggression than pornography featuring a man and a woman.

\section{Previous research on aggression in female/female ( $f / f)$ pornography}

The literature on aggression and violence in intimate relationships between women has been significantly expanding over the last decade (Badenes-Ribera, Frias-Navarro, Bonilla-Campos, Pons-Salvador, \& Monterde-i-Bort, 2015; Rollè, Giardina, Caldarera, Gerino, \& Brustia, 2018). However, to our knowledge, no study has either specifically examined or systematically analyzed aggression in online, mainstream $\mathrm{f} / \mathrm{f}$ pornography. Literature on lesbian pornography, "girl-on-girl" pornography, and comparisons between the two has focused largely on the authenticity of representations (e.g. women's pleasure) but has not addressed physical or verbal aggression (see Hermans, 2012; Jenefsky \& Miller, 1998; Morrison \& Tallack, 2005; Swedberg, 1989). However, to the extent that pornography featuring sex between two women adheres to traditional expectations of feminine behavior, this subgenre would presumably feature less aggression than either $\mathrm{m} / \mathrm{m}$ or $\mathrm{m} / \mathrm{f}$ pornography. Indeed, dominant sexual scripts often frame women's sexuality as passive and receptive (Hayfield \& Clarke, 2012). While anti-pornography scholars often regard men's sexuality as "predatory, exploitative, and dominating," women's sexuality is portrayed as "gentle, nurturing, and egalitarian" (Segal, 1998, p. 47).

To sum up, most previous research did not make explicit statements about the frequency of aggression in same-sex pornography when compared to $\mathrm{m} / \mathrm{f}$ pornography. Still, feminist scholars who hold anti-pornography views appear to suggest that $\mathrm{m} / \mathrm{f}$ pornography should include the largest number of aggressive acts, as many scholars see pornography primarily as a tool for asserting men's dominance over women. In contrast, $\mathrm{f} / \mathrm{f}$ pornography may be expected to include the smallest amount of aggression, as women are often perceived to possess a more passive, affectionate, gentle, nurturing, and egalitarian sexuality. But how accurate are these predictions? How do rates of aggression compare between $\mathrm{f} / \mathrm{m}, \mathrm{m} / \mathrm{m}$, and $\mathrm{f} / \mathrm{f}$ videos in mainstream online pornography?

\section{Is aggression most common in heterosexual pornography? Findings from a content analysis of popular online videos}

As we detail in our methodological chapter (Chapter 3), we sampled the 70 all-time most frequently watched videos from the "gay" category of PornHub (all including a dyad of two men) and the 70 all-time most frequently watched videos from the "lesbian" category (all including a dyad of two women). To ensure comparability, we contrasted these with the 70 top videos from PornHub's general all-time most-viewed list (all including a dyad of a man and a woman).

In Figure 7.1 we present comparisons for measures of aggression/humiliation and affection/pleasure between $\mathrm{m} / \mathrm{f}, \mathrm{m} / \mathrm{m}$, and $\mathrm{f} / \mathrm{f}$ videos. We present 


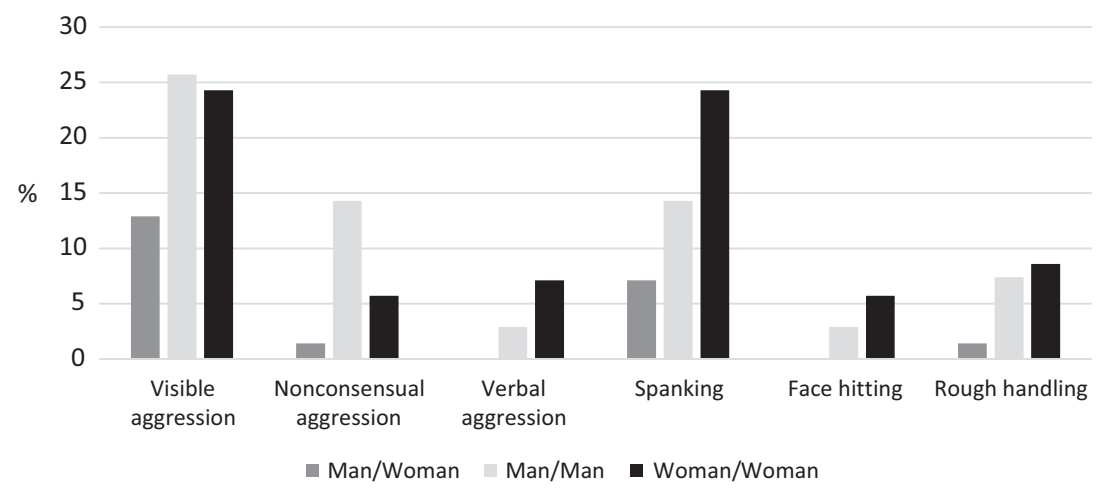

Figure 7.1 Aggression in heterosexual and non-heterosexual dyads $(n=210)$.

both overall measures, differentiated by the definition of aggression (visible physical aggression, non-consensual physical aggression, and verbal aggression) and specific measures for practices that were relatively more common (spanking, face hitting, and rough handling). The figure shows that, in contrast to our predictions, $\mathrm{m} / \mathrm{m}(t=2.314 ; p=0.027)$ and $\mathrm{f} / \mathrm{f}(t=2.01 ; p=0.042)$ videos were more likely to include visible aggression than $\mathrm{m} / \mathrm{f}$ ones. Visible aggression was present in about one-quarter of all $\mathrm{m} / \mathrm{m}$ and $\mathrm{f} / \mathrm{f}$ videos $(25.7 \%$ and $24.3 \%$ respectively), compared with $12.9 \%$ of $\mathrm{m} / \mathrm{f}$ videos. Non-consensual aggression was also most common in "Gay" videos (14.3\%), followed by "Lesbian" videos (5.7\%), while only one video (1.4\%) in the "Heterosexual" sample portrayed such aggression (the difference was statistically significant between the "Gay" and the "Heterosexual" categories: $t=2.890 ; p=0.002)$. Finally, while none of the videos in the "heterosexual" sample included verbal aggression, such aggression was present in a few $\mathrm{m} / \mathrm{m}$ and $\mathrm{f} / \mathrm{f}$ videos, although not frequently $\left(n=2\right.$ and $n=5$, respectively). ${ }^{2}$

As for specific types of aggression, Figure 7.1 shows that the variation in overall aggression has multiple sources. When compared with the $\mathrm{m} / \mathrm{f}$ clips, $\mathrm{m} / \mathrm{m}$ clips were more likely to include acts of spanking, face hitting, and rough handling. However, due to the low frequency of these practices in all categories, these differences were not statistically significant. Videos in the $\mathrm{f} / \mathrm{f}$ sub-sample were also more likely to include acts of spanking $(t=2.847 ; p=0.005)$, face hitting $(t=2.045 ; p=0.043)$, and rough handling $(t=1.981 ; p=0.049)$.

While our focus is primarily on displays of aggression, it is also important to pay attention to displays of affection and pleasure. Here findings seem to be more in line with preliminary expectations. Figure 7.2 shows that samesex videos were more likely to include mutual physical affection and pleasure displays by non-dominant partners. These pleasure displays included smiling, grinning, moaning, and specific affirmative verbal indications (e.g. "yes," "more," or "this feels so good"). While displays of physical affection were present in less than $30 \%$ of the videos in the $\mathrm{m} / \mathrm{f}$ sample, they were present 


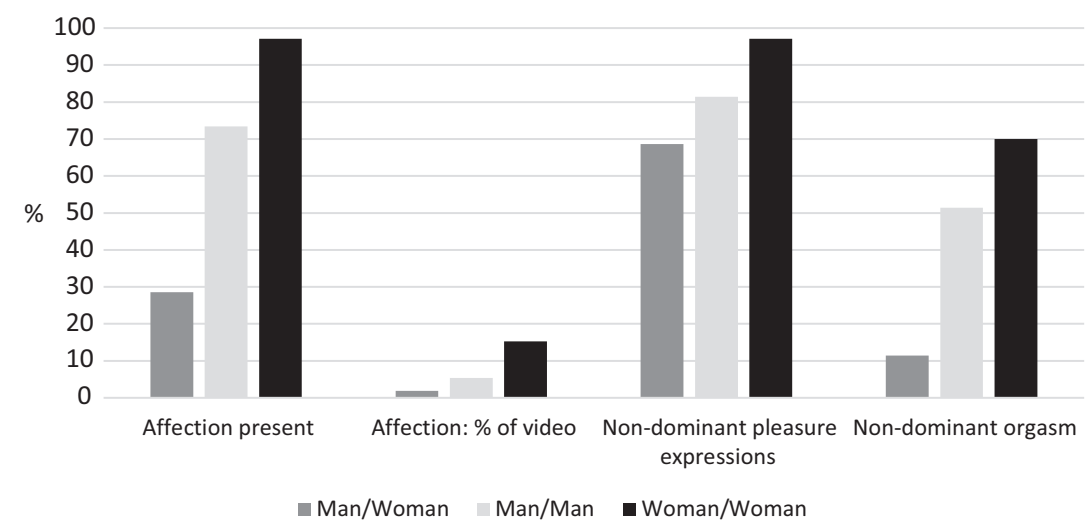

Figure 7.2 Affection and pleasure displays in heterosexual and non-heterosexual dyads $(n=210)$.

in all but one of the videos in the f/f sample $(t=11.830 ; p<0.001)$ and almost three-quarters of the videos in the $\mathrm{m} / \mathrm{m}$ sample $(t=5.572 ; p<0.001)$. Displays of affection were also more prominently featured in the same-sex videos in terms of scripts and plots. This is especially true for $\mathrm{f} / \mathrm{f}$ videos, which portrayed particularly lengthy affection displays, comprising more than $15 \%$ of the total video timespan in the average clip, compared with only about $2 \%$ of the time in the average $\mathrm{m} / \mathrm{f}$ video.

As for pleasure displays by non-dominant partners, Figure 7.2 shows that differences between the $\mathrm{m} / \mathrm{f}$ and the same-sex samples were particularly evident in terms of reaching or portraying a climax. While only $11.4 \%$ of the women in the $\mathrm{m} / \mathrm{f}$ sub-sample reached (or portrayed reaching) a climax, more than half of the non-dominant men in the $\mathrm{m} / \mathrm{m}$ sub-sample reached or performed a climax $(t=5.608 ; p<0.001)$ and $70 \%$ of the non-dominant women in the $\mathrm{f} / \mathrm{f}$ sub-sample also reached or performed a climax $(t=8.721 ; p<0.001)$. These differences in affection, pleasure, and satisfaction remained statistically significant (with odds ratios ranging from 18.49 to 95.93 for the f/f subsample) in a multiple logistic regression analysis, controlling for other factors. ${ }^{3}$ Indeed, these differences in affection and pleasure responses were clearly more pronounced than differences in aggression between the three sub-samples.

\section{What do viewers think about same-sex pornography and aggression?}

We asked our interviewees whether they watched same-sex pornography and, if so, what attracted them to these videos and what they thought about aggression in them. Although the majority of our interviewees identified as heterosexual, most of them said that they frequently watched same-sex 
pornography. In our study, $68.6 \%$ of the self-identified heterosexual male interviewees and $44.1 \%$ of the heterosexual female interviewees said that they regularly watched $\mathrm{f} / \mathrm{f}$ videos (as well as $80 \%$ of the bisexual women who watched f/f videos). In addition, $7.8 \%$ of the heterosexual male interviewees and $9.3 \%$ of the heterosexual female interviewees said that they regularly watched $\mathrm{m} / \mathrm{m}$ videos.

It is clear then that self-identified homosexual and bisexual interviewees were not the only ones who found same-sex videos attractive. We asked heterosexual women who regularly watch lesbian videos about the attraction that these videos hold for them. For most, it was a matter of shifting the focus of the interaction to women's pleasure. For example, Nicole, a 37-year-old communications expert from Canada, said that she liked f/f pornography because of "the aesthetics, the lingerie, things like that. I like it that [the] penis isn't [the] focus." Courtney, a 22-year-old student from Canada, told us that she primarily watches f/f pornographic videos: "I don't really like hetero porn, although I don't identify as bi. I just like [that] there is aggression but it's more subtle between girls. I try to pick videos with more natural stuff." Similarly, Michelle, a 21-year-old student from Canada, said that she watched f/f videos because "I find it's both ways; more female focused. Both partners have the goal of pleasing the other one. Hetero porn is more geared towards man's pleasure and the woman doesn't really matter in that." Elise, a 22-year-old student from the United States, also felt that in $\mathrm{f} / \mathrm{f}$ videos "[w]omen tend to look like [they are] having a good time, not faking it. [These videos are] more gentle, [and there] tends to be more foreplay, versus just penetration." Laura, a 30-year-old married nurse from Canada, summarized these sentiments:

I like it [f/f videos] because it's always pleasurable for [the] female, and it [shows the] female perspective. You can relate. Hetero is most often [showing the] male perspective. Lesbian and threesomes-usually two females and a male-she's finishing in a more relatable way. When I watch a guy cum on [a] girl's face, I don't really relate.

\section{Reflections on aggression in male/male videos}

Women who preferred to watch $\mathrm{m} / \mathrm{m}$ videos cited different motivations. Chloe, a 26-year-old bisexual business entrepreneur from France, thought that "gay porn is very respecting vs. hetero porn. You see an actual relationship between guys. There's a connection; a real one." Julie, a 23-year-old heterosexual student from Mauritius, seemed to have a similar view on $\mathrm{m} / \mathrm{m}$ pornography:

I like [m/m pornography]; it's just the way of interacting, and it's different. It's more intimate looking. Also, you see more male bodies... I find that for women and men it's more aggressive, whereas two men is less aggressive. 
I find that for men and women it's much more coercive and forceful, whereas between two men it's just more... they look more willing.

Others, however, saw things quite differently. Nathan, a 23-year-old food-delivery courier from Canada, said that he liked to watch both $\mathrm{f} / \mathrm{f}$ and $\mathrm{m} / \mathrm{m}$ pornographic videos. He explained his attraction to $\mathrm{m} / \mathrm{m}$ videos despite identifying as heterosexual:

It's really something I don't see often and it's shocking to me, so it's kind of exciting... I'd say [that my] main interest in these types of videos is that there is aggression in them, so I'll search specifically for aggression in gay videos; I'll use keywords [such as] "punishment."

Stephanie, a 40-year-old heterosexual university coordinator, explained her attraction to $\mathrm{m} / \mathrm{m}$ videos in somewhat similar terms: "Gay porn, it's more right to the point; more of a thrill; more my style. It's sex for sex. There's a level of violence [that] I like; it's taboo. More like 'I want your ass' style."

For some viewers, the portrayal of aggressive acts within a more-balanced power dynamic was appealing. This was the case for Esther, a 20-year-old student from Canada, who identified as heterosexual. She declared that she did not like $\mathrm{f} / \mathrm{f}$ videos, but she did often watch $\mathrm{m} / \mathrm{m}$ videos. She explained her attraction to these videos by citing the opportunity to watch aggression in a setting with a more equal balance of power: "I think gay couples' aggression is okay. Two guys can fend for themselves. When it's hetero, [that] is when I'll say it's too much." Cindy, a 26-year-old bisexual retail worker from China, also preferred $\mathrm{m} / \mathrm{m}$ videos:

I prefer homosexual porn because the dynamic is different. I watch a lot more of men with men than women with women. I think that lesbian porn, they are mostly really sweet with each other; it kind of shows what a sexual relationship should be in a good relationship. They are really accommodating of each other. Between men and men, the way they interact with each other, the whole foreplay, what they depict, it's more action-based and quicker. I enjoy those videos more.

Nathan, Stephanie, Esther, and Cindy offer initial insights into our findings regarding the higher levels of aggression in $\mathrm{m} / \mathrm{m}$ pornographic videos. They suggest that at least for some viewers the attraction to $\mathrm{m} / \mathrm{m}$ pornography has to do with their sexually direct script (i.e. having sex simply to have sex) and often also with the higher levels of aggression performed in them. Aisha, an 18-year-old bisexual Canadian student of Pakistani origin, also thought that $\mathrm{m} / \mathrm{m}$ videos were more likely to include aggression. She shared with us her incisive observations on the differences between the three genres:

In gay porn, there is always a bigger "top" vs "bottom" and there is usually more aggression. I definitely see more aggression between two 
men vs. two women. Between a man and a woman, you don't have the concept of like... a twink. With hetero porn, it's just like-she's a woman and therefore she's already a "sub." With two men, you have to decide who is the dominant one. Whoever is more dominant has to be known in gay porn, which means more aggression and force on the submissive one just to establish dominance. In hetero porn it's more of an unsaid male-equals-dominant and female-equals-submissive scenario.

Aisha offers here a highly perceptive potential explanation for the findings we report above regarding the greater likelihood of aggression in $\mathrm{m} / \mathrm{m}$ videos. In her view, this has to do with the need to establish dominance in the sexual relationship through the use of force and aggression. Pierre, a 29-year-old personal assistant from France, who self-identified as gay, also mentioned this difference in the balance of power between $\mathrm{m} / \mathrm{m}$ and $\mathrm{m} / \mathrm{f}$ videos. He claimed that "in gay porn... you're never sure who has actual power. We [gay men] are pretty equal in terms of power. In hetero [porn] it has to be normal that the guy has power." Craig, a 25-year-old production manager from the United States, who identified as bisexual, used to work in the porn industry. He thus provided us with an insider view and was able to offer a complementary explanation for the findings about greater aggression in $\mathrm{m} / \mathrm{m}$ videos:

Men get aggressed most because gay sites have more freedom to [show] extremes... Gay men [videos contain] the most aggression because it's more acceptable to see a guy being aggressed. [He] can get away with way more. Like, consensual issues are less clear in gay videos, especially since people will just say: "Oh, they're two men; of course they want to have sex"... Any aggression towards women has to be soft. It feels like that in mainstream porn, whereas in gay [porn] it can be harder.

In Craig's view then, it is not only the desire to establish dominance. For him, social conventions play an important role in determining the acceptable levels of aggression in all three types of videos. He believes that the common perception that women often find themselves in a sexual scenario despite their will, while men are always willing and eager to participate in sex (which is, of course, in itself a myth), is important in dictating sexual scripts. This perception, he claims, might lead producers and directors to be more cautious when portraying at least some forms of aggression against women in videos, while aggression against men may not be as carefully monitored and censured. Craig also provides a potential explanation for the substantially greater prevalence of nonconsensual aggression in $\mathrm{m} / \mathrm{m}$ videos. For him, this may be the result of norms that see men as always willing and consenting, regardless of the sexual act, even when they appear not to be enjoying the act. 


\section{Reflections on aggression in female/female videos}

As we indicated above, $\mathrm{f} / \mathrm{f}$ videos were very popular with both male and female viewers regardless of their declared sexual identity. Nora, a 22 -year-old woman from Pakistan, who identified as heterosexual, said she liked watching lesbian pornography. She suggested a possible explanation for the greater frequency of aggression in $\mathrm{f} / \mathrm{f}$ videos relative to $\mathrm{m} / \mathrm{f}$ videos:

I think lesbian pornography is really interesting and it's hot, and the things they do are things I tend to be attracted to. I see girls who ride each other; that turns me on. I am very sexual and passionate so lesbian porn, it's such an explicit raw act, and it's kinda dirty and hot... I tend to watch more aggressive lesbian porn, I prefer that, it's the only way they can fuck, they go crazy and they need to. Scissoring is kind of rough to simulate fucking.

For Nora, aggression in $\mathrm{f} / \mathrm{f}$ videos is a form of compensation. As a heterosexual, she views lesbian pornography as "hot" and passionate but somewhat lacking, requiring a flavor of aggression to better simulate "real" fucking. Most interviewees, however, reported that they expected to see less aggression in these videos and most of them also claimed that they observed less aggression. Ishan, a 24-year-old heterosexual student from India, told us that he has "never seen aggression in lesbian porn. Like, maybe a few where one is older, one is step-mom who teaches daughter, but that's not aggression." Michelle, a 21-year-old student from Canada, told us that she encountered less aggression in $\mathrm{f} / \mathrm{f}$ videos but also that "more aggressive lesbian porn is shown as role-play. I haven't encountered one sided aggression. It's always mutual and consensual."

These reported expectations and observations highlight the disparity between viewers' perceptions and actual content, where we found that the most popular $\mathrm{f} / \mathrm{f}$ videos were in fact more likely to include at least some acts of aggression than the most popular $\mathrm{m} / \mathrm{f}$ videos. One potential explanation for this gap may be that viewers overlook aggression in $\mathrm{f} / \mathrm{f}$ pornography and simply fail to see it due to their preliminary expectations. However, some of our interviewees offered a rather different narrative. Hailey, a 21-year-old bisexual student from Canada, told us:

For lesbian porn, it's evident that [the] emphasis is on women. It [aggression] is equally likely [as in $\mathrm{m} / \mathrm{f}$ videos] but [the] dynamics are different when aggression occurs between two women vs a hetero couple. With hetero, it's easy to imagine aggression between men and women. In lesbian porn it's more about [the] pleasure aspects of aggression, because of [the] pleasure the aggression gives, vs. hetero porn where it's more inherent... It's less about pleasure and more about the dominant dynamic. 
For Hailey then, aggression is just as likely to appear in $\mathrm{f} / \mathrm{f}$ videos as it does in $\mathrm{m} / \mathrm{f}$ videos, but the same acts may take on a different meaning. While in $\mathrm{m} / \mathrm{f}$ videos aggression is a way to assert dominance, in lesbian pornography it is more about the pleasure derived from these acts. Patricia, a 26-year-old parttime university administrator from Mexico, identified herself as bisexual and said she enjoyed f/f videos. She also acknowledged that there was aggression in these videos but thought that "[w]ith women it just looks less aggressive. Even if it's spanking, it just seems less aggressive than in [a] hetero situation." Sienna, a 22-year-old bisexual student from the United States, felt similarly that even when aggression does exist in f/f videos, "it feels more like they're on [the] same level; not submissive usually." Nathan similarly referred to the differences in power and dominance to explain why his perception of aggression in $\mathrm{f} / \mathrm{f}$ videos is different: "I think there are differences in aggression. The possibilities aren't the same. Between men and women, there's the raping possibility. It's more like, 'oh yeah, I can really penetrate you against your will."”

Beyond social status and dominance, it appears that the sheer awareness of the differences or similarities in physical strength between the sexual partners influences the way in which viewers decode and interpret acts of aggression that may first appear to be similar in form. Darius, a 34-year-old student from Iran, reflected on the uniqueness of aggression in $\mathrm{f} / \mathrm{f}$ videos: "[Between] two women, the physical power they have is the same. So when they're aggressive, they can perform on each other at the same level." For Darius and other similarly minded interviewees, the assessment of similarities in physical force serves to decipher aggressive acts in $\mathrm{f} / \mathrm{f}$ videos as less threatening and more likely to be mutual. Such readings may help account for the gap between our "objective" coding of aggressive acts and the perception of most interviewees that such acts were less frequent in $\mathrm{f} / \mathrm{f}$ videos. Even when aggression did occur in these videos, interviewees interpreted it differently, saw it as less threatening, and therefore tended to trivialize it or underestimate its occurrence.

\section{Conclusion}

According to some feminists, pornography is, at its core, violence against women, since it sexualizes aggression and portrays women as sexual objects who ostensibly respond favorably to being humiliated and degraded (Dines, 2010; Dworkin, 1981; Dworkin \& MacKinnon, 1988; Langton, 1993; Whisnant \& Stark, 2004). The findings presented in this chapter offer a more complex account. We found considerably higher levels of visible and non-consensual aggression in both $\mathrm{m} / \mathrm{m}$ and $\mathrm{f} / \mathrm{f}$ videos, relative to $\mathrm{m} / \mathrm{f}$ videos. Videos featuring two men contained the highest amount of both visible and non-consensual aggression, while f/f clips had the highest amount of verbal aggression, as well as various forms of physical aggression, such as spanking and choking. These findings therefore challenge the proposition that pornography is primarily about the aggression of men toward women. 
Alongside these high levels of aggression, however, physical affection and non-dominant partners' pleasure displays and climax were far more prominent in same-sex videos than in $\mathrm{m} / \mathrm{f}$ clips. In line with previous research (Séguin, Rodrigue, \& Lavigne, 2018), the low frequency of non-dominant partners' climax in $\mathrm{m} / \mathrm{f}$ clips corresponds with the traditional female sexual script, which dictates that women are and should be more concerned with pleasing their partner than pleasing themselves, while men are and should be focused on pleasure and tension release (Sakaluk, Todd, Milhausen, \& Lachowsky, \& Undergraduate Research Group in Sexuality, 2014). By contrast, non-dominant partners climaxed in over $50 \%$ of the $\mathrm{m} / \mathrm{m}$ clips and $70 \%$ of the $\mathrm{f} / \mathrm{f}$ clips, suggesting more egalitarian scripts at play. But why is aggression more frequent in same-sex videos?

One potential explanation for the higher frequency of aggression (and verbal aggression) in videos featuring two women is that online mainstream pornography, including the "Lesbian" subgenre, often reflects hetero-male fantasies about female sexuality and sexual performativity (Corsianos, 2007; Vincent, 2016; Webber, 2012). Producers of "girl-on-girl” videos, which presumably target primarily heterosexual male consumers, might therefore mirror the scripts found in mainstream heterosexual scenes in an attempt to appeal to heterosexual males (Morrison \& Tallack, 2005; Webber, 2012). Consequently, f/f clips may be depicting aggression in order to abide by the "structural law" of asymmetrical power dynamics central to the mainstream heterosexual script (see Young, 2017). Female aggression in such a context may be a manifestation of what Kalish and Kimmel (2011) describe as the "masculinization of sex."

While these are plausible assumptions, they do not sufficiently account for the substantially higher rate of visible aggression in $\mathrm{f} / \mathrm{f}$ videos. Furthermore, the assumption that heterosexual men are the primary audience for "lesbian" videos is itself problematic. Our interviews with women and men who regularly watch pornography reveal that many women, including many heterosexual women, regularly watch these videos for various reasons. For many of them, the focus on women and their pleasure is a source of attraction. And at least some of them proclaimed that they enjoy some forms of aggression in these videos.

A second, complementary explanation for the preponderance of aggression in the lesbian clips builds on the first and refers to the eroticization of female violence. Similar to the erotic value of lesbianism in hetero-male fantasies (Webber, 2012), the "catfight" is an important part of North American culture (Beck, 1998), eroticizing female-on-female aggression both on- and offscreen (Reinke, 2010; Tambunan, 2018). Given its sexual undertones (Vaes, Paladino, \& Puvia, 2011), it has also become a staple of North American mainstream pornography (Douglas, 1992; Reinke, 2010). In the context of pornography, the "catfight" frames women's aggression toward one another as sexy entertainment for men (Reinke, 2010). Similar to the first explanation, this one is also plausible, and we found some mild support for it in 
interviews with a few of our heterosexual male respondents. However, once again, it should be noted that many of the viewers of $f / f$ videos are women, and most of these did not report aggression to be attractive.

Our interviews suggest a third complementary explanation, one that acknowledges the spirit of the time and potential changes in the way in which both men and women think about the violence of men against women. This kind of violence has become less and less acceptable as feminist conceptions of patriarchal power relations and the illegitimacy of violence in intimate partner relationships have been gaining social acceptance, at least in affluent societies (Messing, Ward-Lasher, Thaller, \& Bagwell-Gray, 2015; Pierotti, 2013). These ideas, in turn, appear to be increasingly permeating the mainstream pornography industry. They render men's aggression against women during sexual acts, particularly aggression without consent, a practice of declining legitimacy and popularity. Conversely, aggression between two women is often perceived by men and women alike as less threatening, as it does not contain in it the traditional notions of patriarchal power imbalance and the potential to turn into an abusive relationship outside of the (virtual) bedroom. Or, as some of our interviewees told us, aggression in $\mathrm{f} / \mathrm{f}$ videos is " just not the same"; it has a different meaning, one that is more about the pleasure derived from the acts than about asserting dominance.

Our findings showing higher rates of both visible and non-consensual aggression in the $\mathrm{m} / \mathrm{m}$ videos compared to $\mathrm{m} / \mathrm{f}$ clips indicate that gendered hierarchies of sexuality are not restricted to heterosexual scripts. "Straight-acting" gay masculinity and a rejection of the feminine are also a prominent feature of gay scripts (see Kendall \& Martino, 2012). These findings problematize "gay-affirmative" conceptualizations of $\mathrm{m} / \mathrm{m}$ pornography, which, perhaps disproportionately, focus on its ability to provide sexual minority men with validation for their sexual desires (Clark, 1991; Tucker, 1991) and to encourage safer sex practices (Levine, 1998; Watney, 1997). The comparative preponderance of aggression in mainstream $\mathrm{m} / \mathrm{m}$ clips indicates a potential assimilation - if not exaggeration — of gay masculinity into hegemonic masculinity.

\section{Notes}

1 We refer here to pornography featuring a dyad of a men and a woman as "male/ female" (m/f), to videos featuring two men as "male/male" $(\mathrm{m} / \mathrm{m})$, and to ones featuring a dyad of two women as "female/female" (f/f). Despite its semantic sex-based limitations (Cante \& Restivo, 2004), we have chosen this terminology given our focus on dyadic scenes and to preclude any assumptions about actors' sexual identities or orientations made by uncritically adopting the labels ascribed to our data (i.e. "Gay" and "Lesbian") by the pornography website from which they were retrieved.

2 The differences in aggression between mixed-sex and same-sex videos remained statistically significant when we conducted a logistic regression analysis, controlling for factors such as the age and race of participants and factors related to the video itself, such as its duration, year of uploading to the PornHub, and 
whether or not the video was an amateur production. Both $\mathrm{m} / \mathrm{m}$ (odds ratio $(\mathrm{OR})=3.14 ; t=2.19)$ and $\mathrm{f} / \mathrm{f}(\mathrm{OR}=2.82 ; t=1.97)$ clips were more likely to include visible aggression than $\mathrm{m} / \mathrm{f}$ clips. This is also the case for non-consensual aggression in the $\mathrm{m} / \mathrm{m}$ sub-sample versus the $\mathrm{m} / \mathrm{f}$ sub-sample, with the former more likely to include videos portraying non-consensual aggression $(\mathrm{OR}=11.29$; $t=2.11)$.

3 We controlled for the age and race of participants, as well as factors related to the video itself, including its duration, the number of views it received, the year it was uploaded to PornHub, and whether the video was an amateur production.

\section{References}

Badenes-Ribera, L., Frias-Navarro, D., Bonilla-Campos, A., Pons-Salvador, G., \& Monterde-i-Bort, H. (2015). Intimate partner violence in self-identified lesbians: A meta-analysis of its prevalence. Sexuality Research and Social Policy, 12(1), 47-59. doi:10.1007/s13178-014-0164-7.

Beck, D. (1998). The "F" word: How the media frames feminism. NWSA Journal, 10(1), 139-153. Retrieved from http://www.jstor.org/stable/4316558

Bishop, C. J. (2015). “Cocked, locked and ready to fuck?”: A synthesis and review of the gay male pornography literature. Psychology \& Sexuality, 6(1), 5-27. doi:10.1080/ 19419899.2014.983739.

Brennan, J. (2016). The "gonzo aesthetic" in gay porn: Fraternity X and sketchy sex. Porn Studies, 3(4), 386-397, doi:10.1080/23268743.2016.1241156.

Bridges, A. J., Wosnitzer, R., Scharrer, E., Sun, C., \& Liberman, R. (2010). Aggression and sexual behavior in best-selling pornography videos: A content analysis update. Violence Against Women, 16(10), 1065-1085. doi:10.1177/1077801210382866.

Brownmiller, S. (1975). Against our will. New York, NY: Simon and Schuster.

Burke, N. B. (2016). Hegemonic masculinity at work in the gay adult film industry. Sexualities, 19(5-6), 587-607. doi:10.1177/1363460716629333.

Cante, R., \& Restivo, A. (2004). The cultural-aesthetic specificities of all-male moving-image pornography. In L. Williams (Ed.), Porn studies (pp. 142-166). London: Duke University Press.

Clark, C. (1991). Pornography without power? In M. Kimmel (Ed.), Men confront pornography (pp. 281-284). New York, NY: Crown.

Cohen, J. N., \& Byers, E. S. (2014). Beyond lesbian bed death: Enhancing our understanding of the sexuality of sexual-minority women in relationships. The Journal of Sex Research, 51(8), 893-903. doi:10.1080/00224499.2013.795924.

Corsianos, M. (2007). Mainstream pornography and "women”: Questioning sexual agency. Critical Sociology, 33, 863-885. doi:10.1163/156916307X230359.

Courtice, E. L., \& Shaughnessy, K. (2018). The partner context of sexual minority women's and men's cybersex experiences: Implications for the traditional sexual script. Sex Roles, 78(3-4), 272-285. doi:10.1007/s11199-017-0792-5.

Currin, J. M., Hubach, R. D., Durham, A. R., Kavanaugh, K. E., Vineyard, Z., \& Croff, J. M. (2017). How gay and bisexual men compensate for the lack of meaningful sex education in a socially conservative state. Sex Education, 17(6), 667-681. doi:10.1080/14681811.2017.1355298.

Dines, G. (2010). Pornland: How porn has hijacked our sexuality. Boston, MA: Beacon Press.

Dines, G., Jensen, R., \& Russo, A. (1998). Pornography: The production and consumption of inequality. New York, NY: Routledge. 
Douglas, S. (1992). Where the girls are: Growing up female with the mass media. New York, NY: Three Rivers Press.

Dworkin, A. (1981). Men possessing women. New York, NY: Perigee.

Dworkin, A. (1987). Pornography is a civil rights issue for women. Journal of Law Reform, 21(1-2), 55-67. Retrieved from https://heinonline.org/HOL/P?h=hein. journals/ umijlr21\&i=63

Dworkin, A., \& MacKinnon, C. (1988). Pornography and civil rights: A new day for women's equality. Minneapolis, MN: Organizing Against Pornography.

Dyer, R. (1994). Idol thoughts: Orgasm and self-reflexivity in gay pornography. Critical Quarterly, 36(1), 49-62. doi:10.1111/j.1467-8705.1994.tb01012.x.

Escoffier, J. (2003). Gay-for-pay: Straight men and the making of gay pornography. Qualitative Sociology, 26(4), 531-555. doi:10.1023/B:QUAS.0000005056.46990.c0.

Fejes, F., \& Petrich, K. (1993). Invisibility, homophobia, and heterosexism: Lesbians, gays, and the media. Critical Studies in Media Communication, 10(4), 396-421. doi:10.1080/15295039309366878.

Foubert, J. D. (2016). How pornography harms: What today's teens, young adults, parents, and pastors need to know. Bloomington, IN: LifeRich Publishing.

Fradd, M. (2017). The porn myth: Exposing the reality behind the fantasy of pornography. San Francisco, CA: Ignatius Press.

Fung, R. (1991). Looking for my penis: The eroticized Asian in gay porn. In Bad Object-Choices (Eds.), How do I look? Queer film and video (pp. 145-168). Seattle, WA: Bay Press.

Hayfield, N., \& Clarke, V. (2012). "I'd be just as happy with a cup of tea”: Women's accounts of sex and affection in long-term heterosexual relationships. Women's Studies International Forum, 35(2), 67-74. doi:10.1016/j.wsif.2012.01.003.

Hedges, C. (16 February, 2015). Pornography is what the end of the world looks like. Retrieved from https://www.truthdig.com/articles/ pornography-is-what-the-end-of-the-world-looks-like/

Hermans, E. (2012). Counter-narratives: Re-evaluating representations of lesbian subject-identities in American popular culture (Unpublished doctoral dissertation). Purdue University, West Lafayette, IN.

Jackson, S., \& Gilbertson, T. (2009). “Hotlesbians”: Youngpeople'stalkaboutrepresentations of lesbianism. Sexualities, 12(2), 199-224. doi:10.1177/1363460708100919.

Jenefsky, C., \& Miller, D. H. (1998). Phallic intrusion: Girl-girl sex in Penthouse. Women's Studies International Forum, 21(4), 375-385. doi:10.1016/ S0277-5395(98)00042-9.

Jensen, R. (2007). Getting off: Pornography and the end of masculinity. Cambridge, MA: South End Press.

Kalish, R., \& Kimmel, M. (2011). Hooking up: Hot hetero sex or the new numb normative?. Australian Feminist Studies, 26(67), 137-151. doi:10.1080/08164649. 2011.546333.

Kendall, C. N. (1999). Gay male pornography/gay male community: Power without consent, mimicry without subversion. In J. Kuypers (Ed.), Men and power (pp. 157-172). Halifax, NS: Fernwood Press.

Kendall, C. N. (2004). Educating gay male youth: Since when is pornography a path towards self respect? Journal of Homosexuality, 47(3-4), 83-128. doi:10.1300/ J082v47n03_06.

Kendall, C. N. (2006). Pornography, hypermasculinity, and gay male identity: Implications for male rape and gay male domestic violence. In C. Kendall \& 
W. Martino (Eds.), Gender outcasts and sexual outlaws: Sexual oppression and gender hierarchies in queer men's lives (pp. 105-130). New York, NY: Harrington Park Press.

Kendall, C. N., \& Martino, W. (2012). Stats please: Gay men as mimics, robots, and commodities in contemporary cultural spaces. In A. Lambert (Ed.), Gendered outcasts and sexual outlaws (pp. 68-84). New York, NY: Routledge.

Kubicek, K., Carpineto, J., McDavitt, B., Weiss, G., \& Kipke, M. D. (2011). Use and perceptions of the internet for sexual information and partners: A study of young men who have sex with men. Archives of Sexual Behavior, 40, 803-816. doi:10.1007/ s10508-010-9666-4.

Langton, R. (1993). Speech acts and unspeakable acts. Philosophy and Public Affairs, 22(4), 293-330. Retrieved from http://www.jstor.org/stable/2265469

Levine, M. P. (1998). Gay macho: The life and death of the homosexual clone. New York, NY: New York University Press.

MacKinnon, C. A. (2018). Sex and violence: A perspective. In P. Searles (Ed.), Rape and society: Readings on the problem of sexual assault (pp. 28-34). New York, NY: Routledge.

Maddison, S. (2017). Comradeship of cock? Gay porn and the entrepreneurial voyeur. Porn Studies, 4(2), 139-156. doi:10.1080/23268743.2017.1304235.

Mahawatte, R. (2004). Loving the other: Arab-male fetish pornography and the dark continent of masculinity.In P. Church Gibson (Ed.), More direct looks, gender, pornography and power (pp. 127-136). London, UK: British Film Institute.

Mellman, W. L. (2017). Beyond the gender binary in sexual scripts? dating and relationships among transgender men and their non-transgender partners (Unpublished doctoral dissertation). Columbia University, New York, NY.

Messing, J. T., Ward-Lasher, A., Thaller, J., \& Bagwell-Gray, M. E. (2015). The state of intimate partner violence intervention: Progress and continuing challenges. Social Work, 60(4), 305-313. doi:10.1093/sw/swv027.

Morrison, T. G., \& Tallack, D. (2005). Lesbian and bisexual women's interpretations of lesbian and ersatz lesbian pornography. Sexuality and Culture, 9(2), 3-30. doi:10.1007/s12119-005-1005-x.

Nelson, K. M., Simoni, J. M., Morrison, D. M., George, W. H., Leickly, E., Lengua, L. J., \& Hawes, S. E. (2014). Sexually explicit online media and sexual risk among men who have sex with men in the United States. Archives of Sexual Behavior, 43, 833-843. doi:10.1007/s10508-013-0238-2.

Ortiz, C. (1994). Hot and spicy: Representation of Chicano/Latino men in gay pornography. Jump Cut, 39, 83-90. Retrieved from https://www.ejumpcut.org/ archive/onlinessays/ JC39folder/gayLatino Porn.html

Pierotti, R. S. (2013). Increasing rejection of intimate partner violence: Evidence of global cultural diffusion. American Sociological Review, 78(2), 240-265. doi:10.1177/0003122413480363.

Pornhub. (2017, January 4). Pornhub's 2016 year in review. Retrieved from http:// www.Pornhub.com/insights/2016-year-in-review

Pornhub. (2019, January 16). Pornhub's 2018 year in review. Retrieved from https:// www.Pornhub.com/insights/2018-year-in-review

Reinke, R. (2010). Catfight: A feminist analysis. Chrestomathy: Annual review of undergraduate research, school of languages, cultures, and world affairs, College of Charleston, 9, 162-185. Retrieved from http://chrestomathy.cofc.edu/documents/ vol9/Reinke.pdf 
Rollè, L., Giardina, G., Caldarera, A. M., Gerino, E., \& Brustia, P. (2018). When intimate partner violence meets same sex couples: A review of same sex intimate partner violence. Frontiers in Psychology, 9, 1-13. doi:10.3389/fpsyg.2018.01506.

Rubin, G. (1993). Thinking sex: Notes for a radical theory of the politics of sexuality. In P. Aggleton \& R. Parker (Eds.), Culture, society, and sexuality: A reader (pp. 100-133). New York, NY: Routledge.

Sakaluk, J. K., Todd, L. M., Milhausen, R., \& Lachowsky, N. J., \& Undergraduate Research Group in Sexuality (URGiS). (2014). Dominant heterosexual sexual scripts in emerging adulthood: Conceptualization and measurement. Journal of Sex Research, 51, 516-531. doi:10.1080/00224499.2012.745473.

Schrimshaw, E. W., Antebi-Gruszka, N., \& Downing Jr, M. J. (2016). Viewing of internet-based sexually explicit media as a risk factor for condomless anal sex among men who have sex with men in four US cities. PloS One, 11(4), e0154439. doi:10.1371/journal.pone.0154439.

Segal, L. (1998). Only the literal: The contradictions of anti-pornography feminism. Sexualities, 1, 43-62. doi:10.1177/136346098001001003.

Séguin, L. J., Rodrigue, C., \& Lavigne, J. (2018). Consuming ecstasy: Representations of male and female orgasm in mainstream pornography. The Journal of Sex Research, 55(3), 348-356. doi:10.1080/00224499.2017.1332152.

Stein, D., Silvera, R., Hagerty, R., \& Marmor, M. (2012). Viewing pornography depicting unprotected anal intercourse: are there implications for HIV prevention among men who have sex with men? Archives of Sexual Behavior, 41(2), 411-419. doi: 10.1007/s10508-011-9789-2.

Swedberg, D. (1989). What do we see when we see woman/woman sex in pornographic movies. NWSA Journal, 1(4), 602-616.

Tambunan, S. M. G. (2018). Shaming the other woman (pelakor): Female catfight as a spectacle in social media. In C. I. Rumimpunu (Ed.), Language in the online $\mathcal{E}$ offline world 6: The fortitude (pp. 306-312). Surabaya, Indonesia: Petra Press.

Tucker, S. (1991). Radical feminism and gay male porn. In M. Kimmel (Ed.), Men confront pornography (pp. 263-276). New York, NY: Crown.

Vaes, J., Paladino, P., \& Puvia, E. (2011). Are sexualized women complete human beings? Why men and women dehumanize sexually objectified women. European Journal of Social Psychology, 41(6), 774-785. doi:10.1002/ejsp.824.

Vincent, I. N. (2016). Pornography: Masquerading as fantasy, producing reality. McNair Scholars Research Journal, 9(1), 135-153. Retrieved from https://commons. emich.edu/mcnair/ vol9/iss1/11/

Watney, S. (1997). Policing desire: Pornography, AIDS and the media. Minneapolis, MN: University of Minnesota Press.

Webber, V. (2012). Shades of gay: Performance of girl-on-girl pornography and mobile authenticities. Sexualities, 16(1/2), 217-235. doi:10.1177/1363460712471119.

Weitzer, R. (2009). Sex for sale: Prostitution, pornography, and the sex industry. New York, NY: Routledge.

Whisnant, R., \& Stark, C. (Eds.). (2004). Not for sale: Feminists resisting prostitution and pornography. North Geelong, AU: Spinifex Press.

Young, D. R. (2017). Gag the fag, or tops and bottoms, persons and things. Porn Studies, 4(2), 176-192. doi:10.1080/23268743.2017.1307138. 


\title{
8 Race myths
}

\author{
Do racial minority women \\ suffer higher rates of aggression?
}

Do minority women suffer higher rates of aggression than do White women in pornographic videos? Which ethnicities are most likely to be cast as the aggressors in these videos? How do racial interactions (same-race and interracial) affect the portrayal of aggression in today's mainstream pornography? And what do pornography viewers think about sexual aggression performed by or received by minority individuals?

Most previous empirical studies on pornographic content have examined women as a monolithic group of victims, paying little attention to ethnic and racial differences among women and among men and to the interactions between them. In particular, the role of race and ethnicity in shaping the practices and principles of the adult entertainment industry has remained under-theorized and under-studied (Bernardi, 2006; Brooks \& Hebert, 2006; Hunter, 2002; Miller-Young, 2010, 2014; Shimizu, 2007; Williams, 2014). Hill Collins (2000) offers that we must conceptualize pornography as an example of the interlocking nature of race, gender, and class oppression. Dines (2006) notes that although racial differences have traditionally been defined and coded through gender, previous feminist analyses of porn have often excluded race as well as other economic and social inequalities. Brooks (2010), who studied the exotic dance industry, also identifies a void in the theoretical analyses of racial and sexual hierarchies within various sex industries.

Indeed, a review of previous systematic analyses of pornographic content shows that most of them have neglected the role of race and ethnicity, with three notable exceptions. Cowan and Campbell (1994), examined 54 rental videos of White and Black performers. They found that interactions between a Black man and a White woman or between a White man and a Black woman included more acts of aggression. Monk-Turner and Purcell (1999) also looked at Black and White performers in 40 videocassettes, finding that Black women experienced more aggression from both White and Black men than was the case for White women. The latter experienced the least amount of aggression when paired with a Black man. Finally, Zhou and Paul (2016) examined 170 Internet videos appearing in the "Asian" category. They found that these videos included a lower mean number of aggressive acts compared with videos from other categories. 
While these three studies serve as important landmarks in our understanding of the role of race and ethnicity in pornographic sexual aggression, they share a number of shortcomings. First, they all defined aggression quite broadly and did not consider the issue of consent in their definition (see more in our methodological section). Second, they used either a convenience or a random sample of videos rather than a sample of more highly watched videos, putting in question the amount of exposure that these videos actually received (and therefore their cultural impact). Furthermore, Cowan and Campbell (1994) and Monk-Turner and Purcell (1999) relied on a sample of rented videos. As we discussed in Chapter 2 and further demonstrated in Chapter 5, rented videos are likely to be significantly different from more recent pornographic materials available on the Web in terms of audience, accessibility, length, production, and oftentimes the content itself. Finally, all three previous studies focus on one visible minority group (Black men and women in the first two studies and Asian women in the third) and do not make comparisons between different minority groups and the various gendered and racial interactions among them.

\section{Theory and research on visible minority women in the media and in pornography}

Previous research and theoretical work on Black women in the media and in the public image highlights Black women as more likely to be the recipients of aggression. The work of Black feminist scholars and activists such as Patricia Hill Collins and bell hooks has outlined and challenged the social images of Black women in America as aggressive, domineering, and unfeminine. Feminist scholars have highlighted the stereotypical portrayals of Black women in various media as mammies, matriarchs, welfare mothers, tragic mulattoes, and Jezebels (Hill Collins, 2000, 2004; hooks, 1992, 1994; West, 1995). These stereotypes both reflect and distort the ways in which Black women look at themselves and the ways in which others view them (Hudson, 1998). The Jezebel image is especially prominent in multiple media representations of Black women, including film, television, and music videos (Bordo, 2003; Brooks \& Hebert, 2006; Emerson, 2002; Hill Collins, 2000; hooks, 1992; Turner, 2011), as well as in pornography (Dines, 2006). This imagery, which originated during slavery, is one of a "bad Black girl," who is seductive, hypersexual, lascivious, promiscuous, and exploits men's weaknesses. Her lewd and wanton behavior, often equated to that of an animal in heat, provokes oppression, sexual exploitation, and sexual assault (including rape), designed to tame her by brutalizing her into submission.

Aggression may also be more common in pornographic videos featuring Black female performers because of Black women's lower status within the sex industry. Brooks (2010) reports less demand for dark-skin exotic dancers, who suffer from stereotypes and marginalization. Consequently, Black dancers are required to work harder for their money and adopt practices 
that are more dangerous in order to remain in the industry. They also report suffering more harassment and violence and a lower sense of security. Similarly, Miller-Young $(2010,2014)$ and Reece (2015) argue that Black women are especially devalued (more so than other women of color) in the porn industry, suffering from lower status, lower demand, and lower salaries. Consequently, they are usually relegated to limited categories and required to perform scenes that are viewed as more degrading, involving a wider variety of sex acts. Indeed, former empirical studies of Black women in pornography reported that they suffer from more aggression compared with their White counterparts (Cowan \& Campbell, 1994; Monk-Turner \& Purcell, 1999).

The literature on Latina and Asian women in the media and in porn is significantly more limited that the literature on Black women (Brooks, 2010). In addition, the representations of both Latina and Asian women remain relatively rare on television and in film (Tukachinsky, Mastro, \& Tarchi, 2015). This absence highlights the possible importance of the pornography industry in shaping and reproducing stereotypes about Latina and Asian women, given the fact that it is one of the few media where women from these groups have relatively higher visibility. Media depictions of Latina women have been somewhat similar to those of Black women, although usually not as threatening (Brooks, 2010; Hill Collins, 2004; Vasquez, 2010). In the few films and music videos where they do receive a more substantial role, they are usually portrayed as exotic seductresses and animalistic hypersexualized spitfires, with an emphasis on their physical attributes, such as breasts, hips, and buttocks (Gomez, 2008; Holtzman, 2014; Molina Guzman \& Valdivia, 2004). No previous study has looked specifically at Latina women in pornography.

The representation of Asian women in the media, and specifically in pornography, has received some scholarly attention. Tajima (1989), who studied the historical images of Asian women in American media, argued that they were mostly depicted using one of two stereotypes: A "Dragon Lady" or a "Lotus Blossom." The first stereotype (Dragon Lady) is of a woman who is sexy, exotic, attractive, and actively seductive but also cunning and deceitful. The second (Lotus Blossom) is of a good, innocent, childlike, docile, passive, and submissive doll, eager to please her man (See also Hagedron, 1997; Nakamatsu, 2005; Uchida, 1998). These stereotypes have also been dominant in pornographic depictions of Asian women (Shimizu, 2007). Zhou and Paul (2016), who compared online pornographic videos that appeared in the "Asian Women" category to other videos, found that the former had a significantly lower mean number of aggressive acts per scene. They concluded that Asian women are treated less aggressively in porn and are less objectified, and therefore that their stereotype is closer to "Lotus Blossom" than it is to "Dragon Lady."

These previous analyses of pornographic materials have often focused primarily on female performers and the acts done to them. Male performers, their identities, and differences among them remain invisible in many of these analyses. Following Dines (2006), we argue that a comprehensive gendered approach must take into account both "fuckers" and "fuckees," as well as their diverging racial and ethnic categorizations. While heterosexual pornography 
almost categorically defines all men as "fuckers" and all women as "fuckees," this may have very different meanings and consequences for White men and women and for minority men and women. We must therefore pay closer attention to the interaction of gender and race/ethnicity and to various couplings between men and women from different racial and ethnic groups.

\section{Do racial minority women suffer the highest rates of aggression? Results from a content analysis popular online pornography}

Figure 8.1 presents results for both aggression and affection, comparing four racial/ethnic groups (each appeared in at least 25 videos): White, Black, Latina, and Asian. Of these, White and Black women were less likely to appear in videos showing visible aggression (about one-third of the videos for each of these groups). While these findings confirm our preliminary expectations for White women, the results for Black women are quite surprising, as the literature often suggests that women from this group suffer higher rates of aggression. More in line with our preliminary predictions, more than half $(51.9 \%)$ of the videos containing Latina women depicted visible aggression. Finally, again in contrast to our expectations and the findings previously presented by Zhou and Paul (2016), aggression was present in three-quarters of the videos containing Asian women, a rate substantially higher than for any other group of women in our study.

Figure 8.1 also shows that videos featuring Asian women were most likely to include non-consensual violence. More than one-third of these videos included non-consensual violence, compared to about $14 \%$ for White women and less than 10\% for Latina and Black women. Finally, in addition to examining aggression, we also explored portrayals of affection between sexual partners. Once again, results were quite surprising. Portrayals of affection toward Latina women were substantially more common (about $22 \%$ of all videos featuring Latina women) than such portrayals in videos featuring White and Black

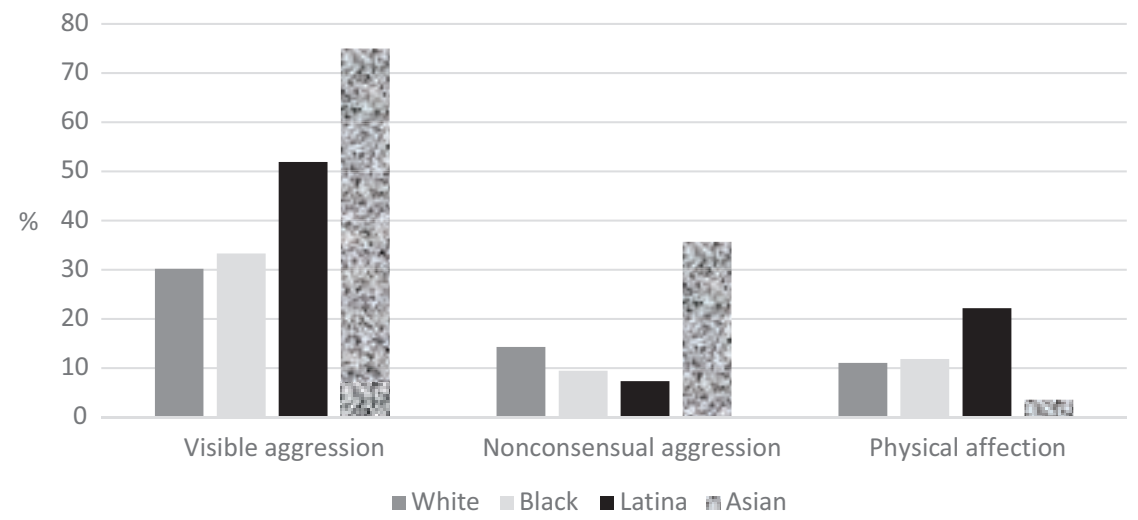

Figure 8.1 Aggression and affection by woman's race. 


\section{Race myths}

women (about 10\% of the videos for each of these groups) and those featuring Asian women (less than $4 \%$ of these videos portrayed displays of affection).

In Figures 8.2 through 8.5, we explore the frequency of three measures of aggression (aggression in the title, visible aggression, and non-consensual aggression) and of affection depending on the racial combination portrayed in the video. First, Figure 8.2 shows that videos including an interaction between a Black man and a White woman were considerably more likely to receive a title that suggests aggression, with more than $40 \%$ of these videos with a title that suggests aggression. This is almost four times higher than the percentage of videos with aggression hinted in title out of all videos where both performers were White (about 11\%). More generally, videos that featured White men were less likely to suggest aggression in their title, with those in which White men were partnered with Asian or Black women particularly less likely to include such titles.

Interestingly, while Figure 8.2 shows that video titles were particularly likely to suggest aggression when Black men were involved, Figure 8.3 shows

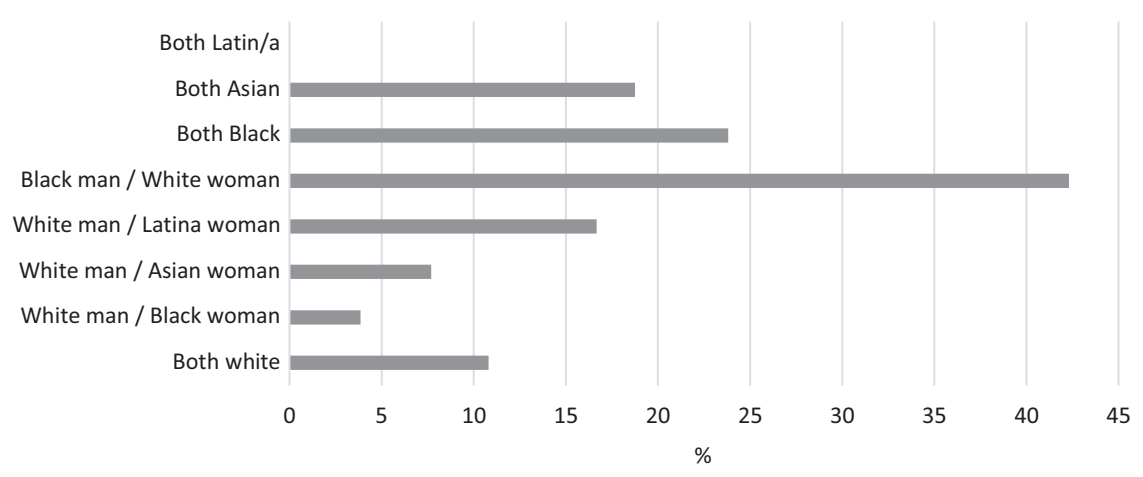

Figure 8.2 Aggression in video title by racial combination.

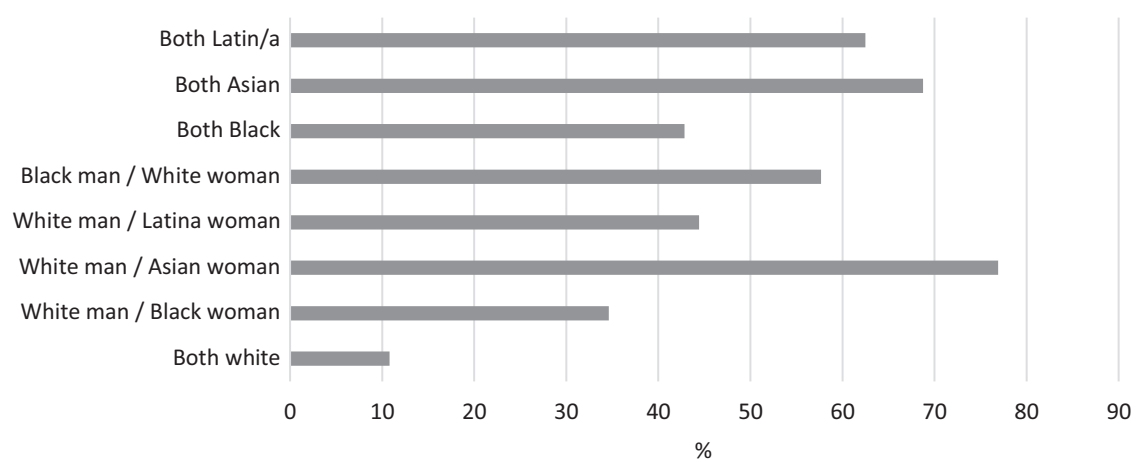

Figure 8.3 Visible aggression by racial combination. 
that this did not translate into actual displays of aggression. It thus may be that what we see here is the work of producers or the individuals who upload these videos to PornHub, as they try to "click-bait" viewers by capitalizing on their stereotypes or fantasies. Visible aggression was present in more than $30 \%$ of the videos in all but one of the ethno-racial combinations we examined-the combination of a White man with a White woman, where only $11 \%$ of the videos included visible aggression. Aggression was most common in videos featuring Asian women, either with a White partner (77\% of all such videos) or with an Asian male partner (69\% of the videos), followed by videos that featured an all-Latin duo (62.5\% of these videos) and those that featured a Black man with a White woman (58\% of these videos).

Next, we examined racial differences in non-consensual aggression. Figure 8.4 shows that such aggression was particularly prevalent in videos featuring Asian women, especially those where the partner was an Asian man. Nearly $44 \%$ of these videos included aggression. It should be noted that non-consensual aggression in such videos was especially widespread not only in videos produced by the Japanese porn industry but also in videos that were produced by the North American industry. Non-consensual aggression was also more common in videos featuring a Black man and a White woman (more than 30\% of these videos included non-consensual aggression) and in those that paired a White man and an Asian woman (23\% of all such videos). Similar to visible aggression, non-consensual aggression was particularly infrequent in videos that featured an all-White duo, with less than 3\% of these videos featuring non-consensual aggression.

Finally, we also looked at expressions of affection by ethno-racial combinations. Interestingly, as shown in Figure 8.5, such expressions were more prevalent in videos that featured Latina women, partnered with either a Latino man (25\% of these videos) or a White man (22\% of these videos). Affection was also present in about $15 \%$ of the videos that included an all-White or an all-Black duo. However, it was rare in videos that included a Black man with

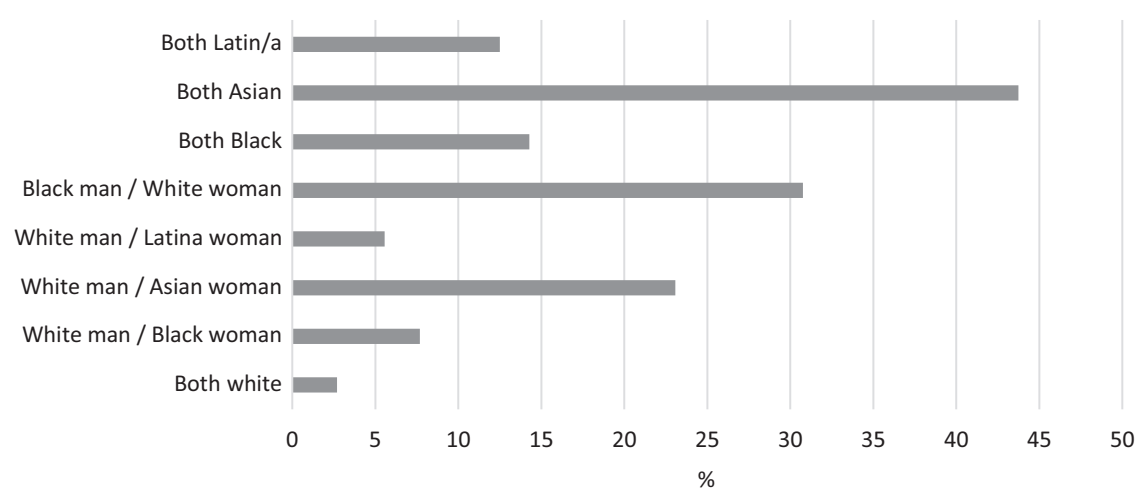

Figure 8.4 Non-consensual aggression by racial combination. 


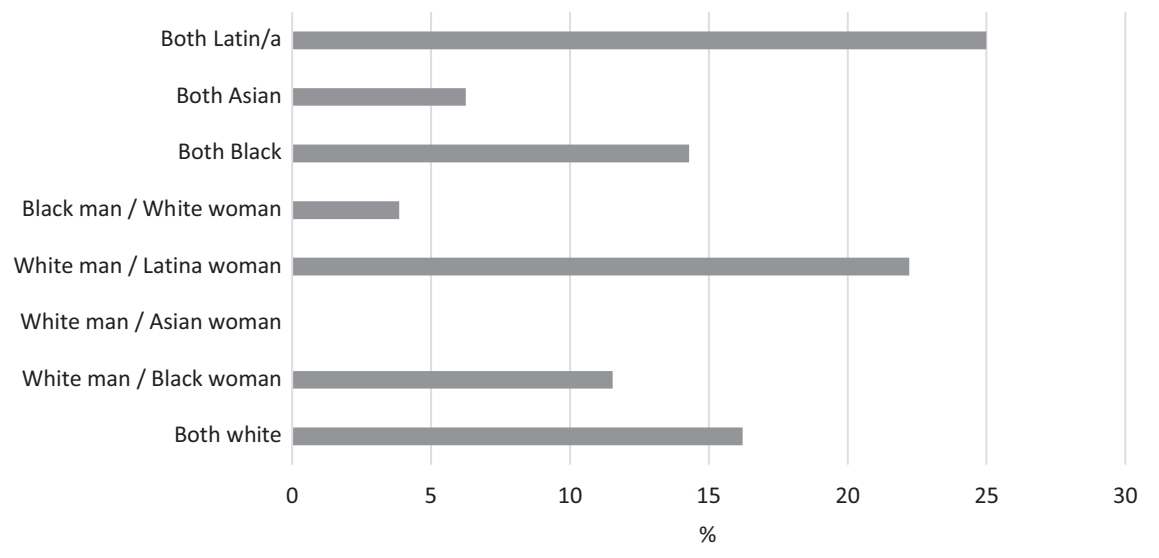

Figure 8.5 Physical affection displays by racial combination.

a White woman (less than $4 \%$ of these videos included affection) and completely absent from videos that featured a White man and an Asian woman.

\section{Pornography viewers' reflections about race, ethnicity, and aggression: interview results}

We asked our interviewees various questions about both their ethno-racial preferences and how they viewed race and ethnicity in relation to aggression in pornography. As we showed in the methodological chapter, about half of the interviewees were White (mostly from Europe and North America), while the other half grew up in non-Western countries or were visible minorities who grew up in (primarily) North America. However, we did not find a strong relationship between viewers' race/ethnicity and their approach to aggression in pornography. The majority of our interviewees claimed that they did not have a particular preference for one ethnicity or another. Some of them, however, did prefer to see performers that were perceived as sharing the same ethnicity as theirs. In particular, some White women preferred to watch videos that featured White performers and some minority men preferred videos that included minority men. Still, with a few exceptions, most of our interviewees did not have a clear preference for only one race or one racial combination and many said that they enjoyed watching various combinations, depending on their mood.

In terms of aggression, interviewees had differing opinions on the race/ ethnicity of performers who were most likely to receive aggression. About half of the interviewees thought that White performers were most likely to be featured in videos including aggression. The other half of our sample believed that women of color, particularly Black and Asian women, were more likely to be the recipients of aggression in mainstream pornographic videos. 
This view was particularly common among non-White interviewees. For example, Janice, a 23-year-old Canadian of Chinese origin, said: "I think Asian and Black women are more likely to be the recipients of aggression." Similarly, Preethi, a 22-year-old female student from India, told us that she saw "a lot of aggression towards Asian women (Mongolian features), they seem to be very submissive... I suspect there's a lot happening towards Black women too." Aisha, an 18-year-old Canadian of Pakistani origin, thought that this was related to cultural stereotypes and perceptions: "I think definitely younger Black women [receive more aggression] because people perceive that it's the culture they come from, so they'd be accustomed to that aggression." In similar fashion, Khalid, a 22-year-old Canadian of Iranian origin, explained why he thought Latina women may be more likely to be featured in videos containing aggression: "Latina women are maybe a little more [likely to be the recipients of aggression]. It's more in their dances and music, so aggression manifests in sex."

There was more agreement among interviewees regarding the race/ethnicity of performers who were most likely to act aggressively. Most of the interviewees thought that Black men were most likely to be cast in these roles, with some believing that White men were equally, or just slightly less, likely to be performing aggressive acts. It should be noted though that most of the interviewees recognized that this was a stereotypical type-cast, rather than an inherent tendency by Black (or White) men to be more aggressive. For example, Kayla, a 21-year-old White Canadian student, told us that "Black men would be portrayed as more violent to propagate racist stereotypes." Similarly, Preethi mentioned that "it's a lot of Black men being aggressive. It's a trope that's being cycled around." Darius, a 37-year-old student from Iran, summarized this sentiment: "It's a little more Black men [performing aggressive acts]. But that's just the director telling them to do things."

A few interviewees found mild aggression in interracial couplings, particularly between a Black man and a White woman, to be arousing. For example, Darius believed that when the video involved a "Black guy and White woman, there are certain acts that are more arousing... I just sometimes enjoy it." William, a 25-years-old Black food attendant from Zimbabwe, agreed that "if it's [aggression between] a Black man and a White woman, it's more arousing." Dean, a 26-year-old White Canadian, said that he liked "seeing an Asian girl with a White male partner. I just think Asian women are very attractive."

However, most interviewees felt uncomfortable about portrayals of aggression in mixed-race couplings. For some it was related to a general dislike of portrayals of aggression. For example, Dominique, a 22-year-old White Master's student from France, said: "It's not about race; it's about acts of aggression." Many others felt that the racial element adds to this discomfort and paints the interaction in degrading colors. Craig, a 25-year-old White production coordinator from the United States, said that he felt "weird seeing a tiny White woman with [a] huge Black dude. Mixed-race scenes, they 
play up race, and I feel weird about it, I prefer it when ethnicities are ignored and it's just about sex." Patricia, a 26-year-old Master's student from Mexico, also felt that "when it's [the] same race, I find it less degrading even if it's aggressive." Tamika, a 20-year-old Black student from Cote D’Ivoire, agreed that "[aggression between a] White man and [a] Black woman [is] more degrading; any White man with a woman of color [is more degrading]." Similarly, Jeffrey, a 23-year-old Chinese student, noticed that "sometimes it's a Black cock, usually labeled as very aggressive acts on White or Asian women. I think it's more degrading." Erin, 28-year-old, unemployed White from the United States, said that "the whole White man and minority woman dynamic... feels gross and degrading, especially if it's aggressive." Finally, Valentina, a 22-year-old White Canadian student, agreed that "if it's [a] White man and a woman of color, more aggressive acts in particular, I'd find it more degrading."

It was not always clear, though, why participants found aggression in interracial combinations to be uncomfortable or degrading, as most of them did not explain this common perception. One possible answer comes from Laura, a 30-year-old White Nurse from Canada:

If there's a clear racial undertone, then it's not comfortable... [O]nce I saw porno of White girl with Black guy calling him the N-word. Even some of Japanese videos, they make girl more innocent/naïve. That's not really okay... I sympathize with them [White women with a Black man] based on Black men's size, but would feel [the] same way if White guys had similar size.

Another possible reason for interviewees' discomfort with interracial aggression in pornography is their awareness of racial exploitation and power relations, and in particular of the history of Black slavery. Elise, a 22-yearold White student from the United States, emphasized the importance of consent (especially in the context of BDSM videos) but felt that without it, "seeing a White dude slapping a woman of color... is more degrading due to colonial history." Similarly, Aisha thought that "when a White man is super aggressive towards a young woman of color, there is something else going on underneath that, and I don't like it. It's a lot more humiliating." Nicole, a 37-year-old White Canadian, who works in the communication industry, agreed that aggression by White men toward Black women could be especially disturbing: "If it's playing on historical contents, like slavery, then yes, that would be worse. [The] idea of making that erotic is disturbing." Donna, a 19-year-old White student from Canada, summarized these common sentiments:

I don't know, I feel so uncomfortable when it's specifically a Black/ White interracial, because I'm so familiar with the history of slavery [and the] criminalization of Black men accused of raping White women. 
I can't shake that discomfort. The slavery dimension still impacts me; it doesn't fit right with me.

Beyond the issue of slavery, interviewees often felt uncomfortable when racial depictions or interracial couplings in pornographic videos echoed real-world conditions of racialized sexual exploitations. For example, Maria, a 20-yearold Canadian student of Hispanic origin, criticized the tendency to depict Black men as aggressive toward White women: "I think it's bad because it emphasizes, in [the] US for example, [that] Black people are killed because they're seen as aggressive." Floriane, a 22-year-old White student from Canada, found videos that corresponded with the South- and East-Asian sex industry and its use by Western men to be disturbing:

I find it more degrading when it plays on things that are realities. Like if you have an older White man and a young Asian girl... If it's set in a Thai brothel with an old White man, if you're imitating anything that has real implications, [it is] appalling to put into [a] porn setting.

Such views echo the tendency among viewers, which we reported in previous chapters (particularly in Chapter 6), to emphasize the importance of pornographic videos as fantasy rather than accurate reflections of the real world. Such tendencies tend to be particularly powerful when the fantasy seems both abusive and likely to occur.

Of note, none of the interviewees mentioned Asian men as more likely to perform aggressive acts. This may seem somewhat surprising considering the findings of our content analysis, which showed that Asian men (invariably cast together with Asian women in frequently watched videos) were in fact the most likely perpetrators of aggression in pornographic videos.

\section{Conclusion}

In this chapter we examined the interaction between gender and ethno-racial categories in online pornographic videos and its association with different measures of aggression and affection. We also explored what viewers of online pornography think about this relationship and more generally about the presentation of race and ethnicity in mainstream online pornography. We found a number of important tendencies, some of them surprising. First, in line with the results of previous studies, as well as the stereotypical public image of Black and Latino men as sexual beasts - violent and hypersexualized-we found that Black and Latino men were more likely to perform aggression in videos when compared with White men. However, while these representations were common, many pornography viewers that we interviewed found them uncomfortable, unattractive, and racist. Most tend to interpret these scripts as originating from producers' and directors' stereotypical choices, rather than from minority men's actual preferences or tendencies. 
More surprisingly, we also found that videos featuring Asian men were significantly more likely to portray male aggression. In fact, this was Asian men were the group most likely to engage in both visible and nonconsensual aggression. This finding appears to contradict common Western media images of Asian men as weak, effeminate, and non-aggressive. However, the videos featuring Asian men were not produced by Western production companies (which almost invariably exclude Asian men from their productions). Instead, all of these videos were products of the Japanese adult entertainment industry, which has unique characteristics that distinguish it from Western pornography. Indeed, this industry includes notable and popular genres that often portray women as victims and men as molesters and abusers, such as reipu (rape), kankin (imprisonment), and chikan (a molester on the train) (Wong \& Yau, 2012). Nevertheless, despite this high involvement of Asian men in videos that portray aggression, our interviews with online pornography viewers reveal that they continue to believe that White and particularly Black men are the ones most likely to commit aggressive acts in porn. Asian men thus remain invisible in pornography, as they are in most Western media artifacts, even when they are clearly present and actively perform.

As for women, the most unexpected finding of our study was that Black female performers were the least likely group of women to suffer from non-consensual aggression and were also more likely to be the recipients of affectionate acts from either White or Black male partners. These findings stand in contrast to the extensive literature on the media representation of Black women (Brooks \& Hebert, 2006; Hill Collins, 2000; hooks, 1992) and more specifically the literature on Black women in the sex industry (Brooks, 2010; Miller-Young, 2010, 2014; Reece, 2015). They also contradict the findings of previous research on rental pornographic videos, which reported that Black women were more likely to suffer from aggression (Cowan \& Campbell, 1994; Monk-Turner \& Purcell, 1999). However, these findings do resonate though with those of Parrott and Titcomb Parrott (2015), who studied television crime-based dramas. They found that White female television characters were, in fact, more likely to be the victims of crime and violence than Black women were. One possible explanation for this surprising finding may be a growing consciousness among many pornography viewers regarding the injustices of slavery and its continuing impacts. Indeed, our interviews with pornography viewers suggest that such awareness makes aggression against Black women, especially by White men, increasingly unacceptable and unattractive.

Finally, while Black female performers were not more likely to suffer from aggression, videos featuring Latina and Asian performers did include a higher rate of aggression compared with those featuring White (or Black) female performers. This finding is especially counterintuitive with respect to Asian female performers, as they seem to stand in contrast with both previous 
literature about the most common media images of Asian women (Hagedron, 1997; Nakamatsu, 2005; Uchida, 1998) and the recent study by Zhou and Paul (2016) on aggression toward Asian women in pornographic videos. Furthermore, this finding cannot be attributed to differing norms in various porn industries, as Asian female performers were likely to suffer from aggression in both Japanese- and Western-produced videos (in fact, even slightly more so in the latter).

It therefore appears that Asian women's passivity and lack of resistance may be perceived as encouragement, and even when they do show discomfort or pain, these are likely to be ignored. This conclusion is in line with Gossett and Byrne's (2002) study of Internet rape sites, which found an overrepresentation of Asian women. It is also pursuant to the arguments of feminist scholars who linked the stereotypes of Asian women as passive doll-like "fucking machines" (Nowrojee \& Silliman, 1997, p. 78) to practices of sexual violence and to greater vulnerability and risk of being mistreated by men who view them as easy targets (Pyke \& Johnson, 2003).

\section{References}

Bernardi, D. (2006). Interracial joysticks: Pornography's web of racist attractions. In P. Lehman (Ed.), Pornography: Film and culture (pp. 220-243). New Brunswick, NJ: Rutgers University Press.

Bordo, S. (2003). Unbearable weight: Feminism, western culture, and the body. Berkeley: The Regents of the University of California.

Brooks. (2010). Hypersexualization and the dark body: Race and inequality among black and latina women in the exotic dance industry. Sexuality Research and Social Policy, 7(2), 70-80.

Brooks, D., \& Hebert, L. (2006). Gender, race, and media representation. In B. Dow \& J. Wood (Eds.), The Sage handbook of gender and communication (pp. 297-317). Thousand Oaks, CA: Sage.

Cowan, G., \& Campbell, R. (1994). Racism and sexim in interracial pornography. Psychology of Women Quarterly, 18, 323-228.

Dines, G. (2006). The white man's burden: Gonzo pornography and the construction of black masculinity. Tale Journal of Law and Feminism, 283, 283-297.

Emerson, R. (2002). "Where my girls at?" Negotiating black womanhood in music videos. Gender \& Society, 16(1), 115-135.

Gomez, L. E. (2008). Manifest destinies: The making of the Mexican American race. New York, NY: NYU Press.

Gossett, J. L., \& Bryne, S. (2002). "Click here”: A content analysis of Internet rape sites. Gender \& Society, 16(5), 689-709.

Hagedron, J. (1997). Asian women in film: No joy, no luck. In S. Biagi \& M. Kern-Foxworth (Eds.), Facing difference: Race, gender, and mass media (pp. 90-103). New York, NY: Routledge.

Hill Collins, P. (2000). Black feminist thought: Knowledge, consciousness, and the politics of empowerment, (2nd Ed.). New York, NY: Routledge. 
Hill Collins, P. (2004). Black sexual politics: African Americans, gender, and the new racism. New York, NY: Routledge.

Holtzman, L. (2014). Media messages, (2nd Ed.). New York, NY: Routledge.

hooks, b. (1992). Black looks: Race and representation. Boston, MA: South End Press.

hooks, b. (1994). Outlaw culture: Resisting representations. New York, NY: Routledge.

Hudson, S. (1998). Re-creational television: The paradox of change and continuity within stereotypical iconography. Sociological Inquiry, 68(2), 242-257.

Hunter, M. L. (2002). "If you're light you're alright": Light skin color as social capital for women of color. Gender \& Society, 16(2), 175-193.

Miller-Young, M. (2010). Putting hypersexuality to work: Black women and illicit eroticism in pornography. Sexualities, 13(2), 219-235.

Miller-Young, M. (2014). A taste of brown sugar. Durham, NC and London, UK: Duke University Press.

Molina Guzman, I., \& Valdivia, A. N. (2004). Brain, brow, and booty: Latina iconicity in U.S. popular culture. The Communication Review, 7, 205-221.

Monk-Turner, E., \& Purcell, C. (1999). Sexual violence in pornography: How prevalent is it? Gender Issues, 17, 58-67.

Nakamatsu, T. (2005). Faces of "Asian brides": Gender, race, and class in the representations of immigrant women in Japan. Women's Studies International Forum, 28(5), 405-417.

Nowrojee, S., \& Silliman, J. (1997). Asian women's health: Organizing a movement. In S. Shah (Ed.), Dragon ladies: Asian American feminists breath fire. Boston, MA: Sutherland Press.

Parrott, S., \& Titcomb Parrott, C. (2015). U.S. television's "mean world" for white women: The portrayal of gender and race on fictional crime dramas. Sex Roles, $73,70-82$.

Pyke, K. D., \& Johnson, D. L. (2003). Asian American women and racialized femininities: "Doing" gender across cultural worlds. Gender \& Society, 17(1), 33-53.

Reece, R. (2015). The plight of the black Belle Knox: Race and webcam modelling. Porn Studies, 2, 269-271.

Shimizu, C. P. (2007). The hypersexuality of race: Performing Asian/American women on screen and scene. Durham, NC: Duke University Press.

Tajima, R. (1989). Lotus blossoms don't bleed: Images of Asian women. In A. W. U. o. California (Ed.), Making waves: An anthology of writings by and about Asian American women (pp. 308-317). Boston, MA: Beacon.

Tukachinsky, R., Mastro, D., \& Tarchi, M. (2015). Documenting portrayals of race/ ethnicity on primetime television over a 20-year span and their association with national-level racial/ethnic attitudes. Journal of Social Issues, 71(1), 17-38.

Turner, J. S. (2011). Sex and the spectacle of music videos: An examination of the portrayal of race and sexuality in music videos. Sex Roles, 64, 173-191.

Uchida, A. (1998). The orientalization of Asian women in America. Women's Studies International Forum, 21(2), 161-174.

Vasquez, J. M. (2010). Blurred borders for some but not "others": Racialization, "flexible ethnicity, " gender, and third-generation Mexican American identity. Sociological Perspectives, 53(1), 45-72.

West, C. M. (1995). Mammy, sapphire, and jesebel: Historical images of black women and their implications for psychotheraphy. Psychotherapy, 32(3), 458-466. 
Williams, L. (2014). Pornography, porno, porn: Thoughts on a weedy field. Porn Studies, 1(1-2), 24-40.

Wong, H. W., \& Yau, H. Y. (2012). The 'real core': The taste of Taiwanese men for Japanese adult videos. Sexualities, 15, 411-436.

Zhou, Y., \& Paul, B. (2016). Lotus blossom or dragon lady: A content analysis of "Asian women" online pornography. Sexuality \& Culture, 20, 1083-1100. doi:10.1007/s12119-016-0375-9. 


\section{Conclusion}

\section{An empirically based account of aggression in pornography}

In this book we sought to provide an empirically grounded review and analysis of current issues related to aggression in pornography. Our goal was to reexamine some of the main narratives about the role of aggression in today's mainstream free online pornography industry and assess their validity. These include the connection between pornography viewing and real-life sexual behaviors (in particular aggressive sexual acts); the frequency and popularity of aggression in mainstream pornography; time-trends in the occurrence of aggression; the intersection of race/ethnicity and sexual orientations with aggression in pornography; and the perceptions and preferences of both men and women who watch pornography online.

In order to provide a sound empirical basis for the discussion, we coded and analyzed 409 videos from PornHub, arguably the most popular pornography website today. We sampled both highly watched videos and random videos as well as videos that expanded the racial and ethnic diversity of our sample and same-sex videos. In our coding, we paid particular attention to the definition of aggression and in particular to the role of consent in this definition. We thus coded both "visible aggression," focusing on the acts themselves, and "non-consensual aggression," which focuses on performers' verbal or nonverbal consent. In addition, we conducted in-depth interviews with 122 online pornography viewers (60 men, 61 women, and one gender-diverse individual). These interviews allowed us to assess not only what is "out there" (i.e. what the content of mainstream pornography looks like) and what viewers are watching but also the ways in which viewers think of and respond to both aggressive and non-aggressive content, what they prefer to watch, and how their viewing might be related to their sex and personal lives.

Many of our findings were counterintuitive, at least for those who are well versed in the current literature (both academic and journalistic) and public debates on pornography. Indeed, our findings frequently dispel some of the most widely held views and misconceptions about the prevalence and role of aggression in today's mainstream free online pornography industry, as well as about the ways in which viewers perceive this aggression. Below we highlight nine of the most interesting and surprising findings coming from our study. We reflect on the ways in which these findings correspond with 
previous notions about the industry and the role of aggression in it, as they appear in the academic literature.

\section{Most pornography viewers separate between fantasies and behaviors}

As we report in Chapters 4 and 6, we found a common disconnect between the preferences of many pornography viewers and their real-life sexual practices. Indeed, many of the viewers who reported enjoying aggression in pornographic videos simultaneously reported that they did not seek or enjoy aggression in their own sex life, or at least did not enjoy some of the acts that they found arousing online. As such, many viewers perceived online pornography as a realm where certain fantasies, including fantasies about aggression and domination, could be safely explored. Furthermore, most of our interviewees believed that watching pornography did not really have a meaningful or lasting impact on their lives.

It is certainly possible that at least some of our interviewees were not fully aware of the actual effects pornography has had on them. It may also be that they were either consciously or unconsciously trying to minimize or trivialize these effects, seeking to show that they are in control of their watching habits or trying to demonstrate that regular viewing is harmless. However, it also could be that many frequent viewers indeed gradually become "immune" or desensitized to the effects of pornography or are able to compartmentalize them, as some of our interviewees have suggested. It appears that many of them develop "porn literacy" and come to view and experience pornography as a source of entertainment and fantasy, clearly separate from their sexual interactions with others and generating little influence on these interactions.

\section{Viewers emphasize consent and mutual pleasure when reflecting on aggression}

In our methodological chapter (Chapter 3) we outlined the debate around the very definition of aggression in pornography. We described the most common practice in content analyses of sexually explicit materials-adopting a broad definition for aggression, one that includes any purposeful action that is determined by researchers as causing harm to another person. Under this definition, practices such as spanking, hair pulling, or bondage have commonly been considered as aggressive, regardless of whether performers expressed consent or pleasure. Recognizing the potential importance of consent in these practices, our study has been the first to adopt two separate definitions for aggression and analyze videos accordingly. First, following most previous content analyses, we coded "visible aggression," focusing on the apparent intent to cause harm or pain, even if it was mild. But we also coded "non-consensual aggression," following a definition that takes into account 
the concepts of consent and instrumental aggression (aggression designed to elicit pleasure). In addition, we also differentiated in analyzing our interviews between viewers' responses to and preferences for "milder" and "harder" acts of aggression.

It is important, however, to note where most of the viewers we spoke with stand on this definitional question. As we show in Chapter 5, many of the acts that content analyses of pornography typically consider to be aggressive (e.g. hair pulling and spanking) were not considered to be aggressive by the large majority of viewers with whom we spoke. Instead, viewers cited consent (both verbal and visual) as the primary and most important signal for whether or not they viewed an act as aggressive. Moreover, consent was deemed crucial even by viewers who found aggression to be arousing. Of note, many viewers admitted that consent in pornography is not always easy to determine. Many were also aware of the fact that even when performers clearly express consent or even enthusiasm about an act they may be simply performing a role rather than truly enjoying the act. Still, despite this complexity, the fact that viewers actively seek expressions of both consent and pleasure shows that the large majority of them do not find representations of aggression arousing unless they believe that both sexual parties enjoy it.

\section{Most videos do not contain aggression}

Another common misconception that our findings help disprove is the one holding that aggression in mainstream free pornography is very prevalentalmost omnipresent (Bridges, Wosnitzer, Scharrer, Sun, \& Liberman, 2010; Dines, 2016; Sun, Wosnitzer, Bridges, Scharrer, \& Liberman, 2010). In Chapter 5 we showed that most free online pornographic videos do not contain aggression, even under the most inclusive definitions of aggression, which include acts that many of our interviewees did not think of as aggressive. When adopting more restrictive definitions of aggression, ones that consider the role of consent and instrumental acts, aggression is even less common, appearing in under $10 \%$ of the randomly sampled videos on PornHub and in less than $2 \%$ of the most frequently watched videos. At the same time, displays of pleasure and affection were very common, appearing in most videos.

It is important to discuss here the disparity between our findings and those put forward by one of the most frequently cited studies on aggression in mainstream pornography, conducted by Bridges et al. (2010). They reported that about $88 \%$ of the videos in their study included physical aggression. However, even when we adopted an identical definition for physical aggression to the one used in their study as well as employing an identical sampling strategy (examining the most popular videos), we found that only $12.9 \%$ of the all-time most highly watched videos on PornHub contained physical aggression. How can we account for such a large discrepancy? One potential explanation is the difference in media-rented videos vs. free online pornography. However, it is not at all obvious that these two media would have 
such large discrepancies in their portrayal of pornography. After all, tube sites such as PornHub are typically aggregators of different links and clips, which are very often pirated or stolen (Tarrant, 2016). Therefore, Dines (2016) had suggested that since tube sites proffer for free porn that was produced for sale, the analysis by Bridges et al. on rented and purchased porn videos is likely to also reflect the content of free porn sites. We found that this was not the case.

We therefore need to also consider other explanations. One such explanation may be that the viewership of online pornography is likely different in some ways from the viewership of rented videos. The former tends to be more diverse, including for example more women, more gender and sexual minorities, and a more diverse group of men (Tarrant, 2016), who in turn may prefer to watch less aggressive content. Another possible explanation may have to do with the content that is uploaded online and the content that tube sites choose to censure. First, it could be that users who upload clips and video parts are less likely to upload scenes that contain aggression. In addition, moderators of tube sites may be more likely to censure pornography that includes aggression. This explanation received some support in interviews that we conducted with three former employees of such sites. These former employees argued that at least some aggressive contents were indeed being censured or altogether removed from websites. Finally, the differences between our findings and those of previous research may also reflect changes over time in pornographic materials. We expand on these potential changes in the next section.

\section{Mainstream pornography is not becoming increasingly aggressive}

A final possible explanation for the discrepancy between our own findings about the prevalence of aggression and those of some previous research may be related to another one of our major findings. As we also report in Chapter 5 , we found no support for the common claim that mainstream pornography is getting "harder and harder" with time (Fradd, 2017; Picker \& Sun, 2008; Sun et al., 2010). We did not find a significant temporal increase in videos that depict various types of aggression, but we did find that the average video in later years contained shorter segments depicting aggression. We further observed a moderate reduction in the frequency of videos that contained non-consensual aggression as well as in the prevalence of videos that received a title suggesting aggression. As the analysis by Bridges et al. was conducted on a sample of the most frequently rented videos in 2005, part of the discrepancy between our findings and theirs may therefore be attributed to this general tendency toward fewer depictions of aggression in the most popular mainstream pornographic videos.

The fact that pornography is not becoming increasingly aggressive also has implications for the commonly held societal perception of pornography viewing as an addiction. Those who maintain this perception often contend 
that similar to drug addicts, who consume higher doses once they are unable to reach the same high as with the previous dose, many pornography viewers also become desensitized to aggression. Viewers would therefore be more likely to seek aggressive and demeaning contents once the thrill and excitement previously achieved by traditional "vanilla" videos diminishes (Dodige, 2007; Hilton \& Clark, 2011; Paul, 2010). Our finding that the ratio of videos containing aggression did not increase over the years (and by some measures it actually decreased) provides no support for such arguments about pornography as a widespread addiction with progressive desensitization.

We also did not find any consistent support for a pattern of desensitization and gradually seeking more aggressive materials with time in our interviews with pornography viewers. While some interviewees reported that they indeed began to explore more aggressive materials over the years, others reported the reverse pattern. Once the novelty wore off, they felt "overwhelmed" or "saturated" with aggression and preferred to be exposed to it less frequently. These accounts correspond with those reported by Loftus (2002), who found a parallel tendency in his interviews with male pornography viewers. Similar to our own interviewees, Loftus' interviewees did not report gradually gravitating toward increasingly more violent contents. Most of them either stuck with what they liked initially, investigated harder contents but came back to those they preferred at first, or completely lost interest in aggressive depictions.

\section{Most viewers prefer to watch less aggression}

It is not only that popular mainstream pornography is not becoming more aggressive. We also found that most viewers do not show a preference for aggressive materials. In Chapter 6 , we showed that videos that contained aggression, in particular non-consensual aggression, were significantly less likely to be viewed when compared with videos that did not contain aggression. Conversely, videos that included female performers expressing pleasure and reaching a climax were more likely to receive a larger number of views. Our examination of viewers' ratings for videos that do not include aggression provides further support for the idea that viewers do not prefer aggression. Videos that portrayed non-consensual aggression were significantly less likely to receive favorable ratings from viewers, while those portraying women's pleasure and ecstasy were more likely to received favorable ratings.

Our interviews with pornography viewers offer additional support for the findings coming from our content analysis of videos. About $20 \%$ of our interviewees reported actively seeking aggressive content in the pornography that they regularly watch. However, most viewers preferred not to encounter aggressive materials on a regular basis and desired to see fewer aggressive acts in the videos available to them. In fact, even most of the viewers who did report being aroused by aggressive content said that they would have liked to see less of it offered in the majority of the videos. They said that they could 
easily get to it if they wished and therefore did not feel the need to see it in the videos most readily available to them.

Clearly, then, although aggression is not as common in most mainstream pornography as previous research has suggested, even the amount of aggression that currently exists in mainstream videos is superfluous for most viewers. To the extent that these preferences represent a common sentiment among pornography viewers (the triangulation of interviews and viewers' rankings of videos on the Web gives us some confidence that they do), one might see this as positive news. In particular, scholars and activists who write and speak about the pernicious effects of aggression in pornography, such as its potential links to violence against women (DeKeseredy \& Hall-Sanchez, 2017) and an increased acceptance of rape myths (DeKeseredy, 2015) might find these preferences encouraging. The majority of mainstream viewers appear to reject prevalent depictions of aggression and degradation, particularly from videos that include long sequences of such practices or non-consensual aggression. Depending on the responsiveness of producers to consumer preferences, we may therefore expect the process of reduction in the distribution and prominence of material featuring non-consensual aggression to continue.

\section{Women, not men, are relatively more likely to express interest in aggression}

This was perhaps the most counterintuitive finding of our study. The findings we report in Chapter 6 show that while most viewers preferred nonaggressive videos, this tendency was especially prominent among men. Almost all of our male interviewees (93\% of them) declared that they would not like to see more aggression in pornography. However, this was the case for only about two-thirds of the women we interviewed. Men were also more likely to report not enjoying any sort of aggression in videos $(60 \%$ of the men) and in particular not enjoying harder forms of aggression (83\% of the men) and non-consensual aggression (almost all of the men). In contrast, women were more likely to find at least some forms of aggression in pornography arousing (nearly two-thirds of the women we interviewed), and many of them also reported that they found harder forms of aggression arousing (almost $40 \%$ of the women).

Our findings for men's preferences largely echo those reported nearly 20 years ago by Loftus (2002), who also interviewed male pornography viewers. Most of these men stated that they did not like depictions of domination or aggression against women. They also testified that they did not find the male performers to be suitable role models and did not want to imitate them. Loftus also found that it was important for his male interviewees that the female performers in the videos seemed to be enjoying themselves. His interviewees said that they much preferred it when the female performers were notably involved and interested in the sexual acts rather than merely serving the desires of male performers. We found very similar patterns in the current study. 
Our findings regarding the preference for aggressive content among many women seem even more surprising. Much of the critical literature on pornography has been focusing on male viewers and their seeming preference for (sexual) domination over women as the driving force behind the spread of aggressive and humiliating acts in mainstream pornography. Some have even suggested that men enjoy watching aggression and humiliation of women mainly because these acts celebrate the tension and thrill derived from sexualizing gender inequalities (Dines, 2006, 2010; Dines, Jensen, \& Russo, 1998). Our finding that female viewers were in fact more likely to enjoy such scenes (particularly those depicting harsher aggression) poses a challenge to these notions. Of note, we should exercise caution when generalizing these patterns to all female and male viewers of pornography, as our sample is by no means fully representative of this population. Still, to the extent that we were still able to capture an actual tendency among viewers, we may question both the assertion that pornography is violence against women and the assertion that this is primarily driven by the preferences of male viewers and producers.

One possible challenge to our findings may be the suggestion that the women we interviewed have simply adopted or "bought into" men's fantasies about sex and sexuality, as these are packaged and marketed by the pornography industry. That is, it is possible that the women in our study who showed partiality for aggression have gradually been conditioned (or "brainwashed") into thinking that this is what "real" passionate sex looks like, with the patriarchal norms of the pornography industry shaping their fantasies and sexual tastes (see for example Foucault, 1992). However, this was not our impression when talking to these women. Many of them self-identified as feminists and proclaimed to believe in gender equality and women's right to experience sexual pleasure. Most of the women who reported enjoying aggression appeared to be attracted to the aspects of losing control, being dominated, and potentially deriving greater pleasure from the mix of pleasurable and painful experiences. Furthermore, many of the women we interviewed (and also many of the men who reported being aroused by aggression) clearly distinguished between their desire to watch aggression in pornography and their desire for fewer or no aggressive practices in their own sexual relationships. Thus, as we noted earlier, the attraction was often to the fantasy of aggression rather than to its application and emulation.

\section{Viewers overwhelmingly prefer videos in which female performers express pleasure}

In addition to our insights about aggression, the research findings also indicate a near-universal preference by viewers for videos showing women's pleasure in pornographic videos. Our content analysis showed that videos that included displays of affection and women's pleasure received more views and significantly higher ratings from viewers. Complementing this tendency, our interviewees overwhelmingly emphasized the importance of seeing pleasure 
displays from the performers in pornographic videos. Many further confided that in the absence of such displays they did not find videos arousing. These findings are again consistent with those of Loftus (2002), who found that it was important for his male interviewees that the female performers in the videos seemed to be enjoying themselves. The men in his study also said that they much preferred it when the female performers were enthusiastically involved and invested in the sexual act, an inclination that we also found among men in the current study.

Still, one might certainly question the veracity of pleasure displays in pornographic videos. Some feminist writers have argued that mainstream pornography is made exclusively for men and creates a sexual world centered on men's pleasure (Fitzgerald \& Grossman, 2017; Paul, 2005). Within this world, women's displays of pleasure are rarely (if ever) genuine; they follow a script and serve merely as a testimony of men's prowess and success in eliciting pleasure from their sexual partners, with little regard to consent or authenticity (Dines, 2003). Columnist Joan Smith (2013) expressed this idea in an article published in The Independent:

I have always tried to make a distinction between sex which was enjoyed by both parties and sex-and-violence. These days the latter predominates and the idea that most Internet porn has anything to do with women's sexual pleasure is laughable.

Our findings problematize such contentions. On the one hand, it is true that even if women in pornography perform displays of pleasure and climax, this is often scripted. Interviews with performers in the porn industry (Bauer \& Gradus, 2015; Miller-Young, 2014; Wagoner, 2012) and behind-the-scenes documentations (Bauer \& Gradus, 2015; Sun et al., 2010) often reveal the scripted nature of these interactions, dictating that both female and male performers (in particular the former) display ecstatic pleasure throughout the videos. Yet, following McKee (2005, 2015, 2016), we argue that when analyzing the content of videos, it is important to pay attention not only to the subtext, but also to the text itself. The fact that women display pleasure in the large majority of videos, and that the majority of these pleasure displays do not directly follow aggressive acts, conveys that women's sexual pleasure is important. And indeed, it is exceedingly important to the viewers themselves. It therefore appears that viewers are often willing to suspend their disbelief. They "buy" into the act and enjoy displays of pleasure despite their awareness of the fact that performers often fake such displays. Indeed, interviewees often mentioned the importance of the performance looking genuine and their dislike for performances that seemed fake and were not convincing enough.

It is also important to note that consent in pornographic videos (and in sexual interactions more generally) is rarely completely free. Indeed, consent is embedded in a larger context of gendered power relations and normative expectations regarding the role and performance of men and even more of 
women in the pornography industry (and in other social interactions). However, as McKee (2015) argues, it does not logically follow from this premise that consent is unimportant. Similarly, we argue that pleasure displays, even when scripted and embedded within a set of role expectations, are nevertheless meaningful. They convey a message that women's pleasure is an important part of most sexual encounters and that women are entitled, in fact expected, to experience sexual pleasure. Such expectations are, of course, not without problems of their own. Both female and male viewers may feel pressures to perform certain acts and exhibit pleasure even when their actual experiences do not conform. Still, we found no support for the notion that mainstream pornography is entirely focused on men's pleasure and completely ignores women and their needs, desires, and entitlement to pleasure.

\section{Popular same-sex videos contain more aggression than popular heterosexual videos}

Another surprising finding, reported in Chapter 7, was the considerably higher levels of visible and non-consensual aggression in both male/male and female/female videos, relative to male/female videos. Videos that featured two men had the highest amount of both visible and non-consensual aggression, while those that featured two women had the highest amount of verbal aggression, but also frequently other forms of physical aggression, such as rough handling and choking. These findings once again challenge the proposition that pornography is primarily about the aggression of men toward women. However, we also found that physical affection, as well as non-dominant partners' pleasure displays and climax were much more prominent in same-sex videos than in $\mathrm{m} / \mathrm{f}$ clips.

Our interviews help in shedding some light on these remarkable findings. They suggest that for many viewers, overt violence by men against women is often perceived as unacceptable and offensive, particularly given the clear differences in physical strength between the sexes. However, aggression between two women or between two men is often perceived by both men and women as more symmetric and therefore less threatening and offensive. The reason for this is that such aggression does not contain in it the traditional notions of patriarchal power imbalance and does not appear to carry the same degree of risk to turn into an abusive relationship outside of the (virtual) bedroom. As such, some viewers suggested that they were able to focus more on the pleasure derived from the acts instead of on the assertion of dominance and the potential negative social and interpersonal effects of such representations.

\section{Videos featuring women of color do not always contain more aggression}

Finally, in Chapter 8, we showed that common ideas about racial hierarchies in pornography and the ways these hierarchies shape practices of aggression are 
sometimes misguided. We found a complex relationship between gender, race/ ethnicity, and aggression in pornography, where aggression depends not only on the racial identity of female performers but also on that of male performers and the interaction between race/ethnicity and gender in any given video.

Previous research has reported that Black women are marginalized in the media and specifically in the sex industry (Brooks, 2010; Miller-Young, 2010, 2014; Reece, 2015). Former studies on rental pornographic videos in the 1990s also suggested that Black women were more likely to be the recipients of aggression from both Black and White men (Cowan \& Campbell, 1994; Monk-Turner \& Purcell, 1999). However, we found that Black female performers were actually the least likely racial category of women to be on the receiving end of non-consensual aggression and were also more likely to be the recipients of affectionate acts in online pornographic videos. Our interviews with porn viewers provide one possible explanation for this counterintuitive finding - the growing consciousness among many pornography viewers about the historical injustices of slavery, as well as current racial inequalities, which made them uncomfortable with displays of aggression against Black women, especially by White men.

Conversely, videos featuring Latina and Asian female performers did include a higher rate of aggression when compared with those featuring either White or Black female performers. In particular, we found a significantly higher amount of aggression toward Asian performers, which could not be explained simply by diverging industry norms, as it appeared in both Japanese- and in Western-produced videos. This finding contradicts both previous literature about the most common media images of Asian women (Hagedron, 1997; Nakamatsu, 2005; Uchida, 1998) and the results of a recent study by Zhou and Paul (2016) on aggression toward Asian women in pornographic videos. We offered in Chapter 8 that these patterns may stem from the common perception of Asian women as passive "fucking machines" (Nowrojee \& Silliman, 1997, p. 78), which renders them more susceptible to being mistreated and allows producers, male performers, and viewers to ignore their discomfort or pain. The overrepresentation of Asian women in the service and care industries in the West (particularly in North America) may also contribute to their image as complaisant women, eager to please, and therefore more willing to endure painful sexual acts.

\section{Tying it all together: Toward a nuanced and empirically informed debate about aggression in pornography}

As we have demonstrated throughout this book, the debate about the pornography industry and the role of aggression in it has often been dominated by crude binary arguments that are largely based in ideological positions. These positions greatly influence the contentions of both supporters and critics, the facts that they choose to cite or emphasize, and even the definitions and methodologies they adopt in studying pornography. 
On the one hand, naïve views of pornography paint it as a harmless form of entertainment. In this view, performers freely choose to participate and exercise complete control over their body and the scenes and practices in which they choose to partake. They thus fully consent to the sexual activities in which they participate and even enjoy them, while also earning considerable sums of money and at times even fame, allowing them to leverage their status as porn stars and their brand name in order to increase their revenues. Viewers, on their part, are, in this simplistic view, always wellinformed and recognize that pornographic videos are merely a fantasy rather than depictions of real sex. Therefore, they are not negatively influenced by depictions of aggression or degradation. As such, pornography serves not only as a harmless form of entertainment. It also offers a source for more authentic and realistic sexual education, a medium through which one can safely explore sexual fantasies and a social safety valve for sexually frustrated individuals.

While this simplistic view is rarely fully articulated in the academic literature on pornography, it is quite prevalent in everyday public discussions and is often promoted by many in the industry itself (Picker \& Sun, 2008). And yet, multiple studies have shown the many problems with these assumptions. Some have focused on performers, showing that while most are not forced into the industry and some exercise varying degrees of control over their careers (Miller-Young, 2014), many come from lower socioeconomic backgrounds, where economic opportunities are scarce. Once in the industry, many performers have limited control over the sexual scenes and many of them suffer from long-term negative impacts, including traumatic experiences and a stigma that is particularly hard to shake off, limiting their future opportunities (Bauer \& Gradus, 2015; Ketcham, 2014; Voss, 2015; Wagoner, 2012). As we have shown in Chapter 4, arguments about pornography's complete lack of negative effects on viewers have also been widely refuted by a wide range of empirical research (particularly research on pornography that includes aggression).

On the other hand, many of the accounts by scholars who are fiercely critical of the pornography industry have also been problematic and lacking nuance. Over the years, some radical feminists have viewed pornography per se as violence against women, an industry that fosters sexual aggression, misogyny, and rape myths (Brownmiller, 1975; Dines, 2010; Dworkin, 1989; Dworkin \& MacKinnon, 1988; Russell, 1988). These accounts often emphasize the alleged ubiquity of aggression in videos and the assumed growing rate of aggressive pornography. Some have even suggested that the pervasiveness of aggression and degradation in today's industry testifies to widespread misogyny. For example Gail Dines, one of the most prominent, vociferous, and eloquent anti-pornography scholars, spoke about aggressive acts in pornographic videos during an interview with The Guardian, arguing that "[to] think that so many men hate women to the degree that they can get aroused by such vile images is quite profound" (Bindel, 2010). 
However, many of these contentions about the wide spread of aggression in today's pornography have been based on questionable evidence. Authors and journalists have frequently been citing the findings of a single study (Bridges et al., 2010). However, while this study was rigorous and conducted in a professional manner, it analyzed a form of media (rental videos) that by now has almost been rendered obsolete and relied on a priori definitions of aggression, which neither reached consensus in the literature nor were based on the common perceptions of pornography viewers. In the present study we presented evidence for a substantially different picture regarding both the prevalence of aggression in today's pornography and the alluded trend toward higher rates of aggression with every passing year. We also questioned the assumption that aggression is limited to or at least most common in videos including a man and a woman, thus problematizing the view of pornography itself as violence against women.

We thus argue that anti-pornography scholars and journalists may have been confounding supply — what some mainstream pornography looks likeand demand - what most viewers actually want to watch. Indeed, while some viewers (both men and women) undoubtedly enjoy performances of aggression and degradation of women (and sometimes men) and find them to be arousing and exciting, our study provides no support for the claim that the majority of viewers - and in particular the majority of male viewers-seek such images or prefer to be exposed to them. Instead, most viewers clearly preferred videos that included displays of affection and pleasure. And while the literature often focuses on aggression, such displays of affection and pleasure were in fact much more prominent and lengthy in the majority of the mainstream videos. Furthermore, even those interviewees who found gender inequalities in videos appealing - most of these, as we discussed above, were women-were primarily attracted to the aspects of domination or giving up control rather than to aggression for the sake of aggression.

We thus conclude this book by calling for a more nuanced discussion of aggression and of the pornography industry more generally, one that is better informed by the empirical data and more carefully considers the complexity of both concepts and personal views. This discussion should not ignore the negative aspects of the pornography industry and its documented harmful effects. But it should also not be dedicated to the vilification of this industry, while ignoring both recent developments that make it more diverse and multifaceted and the complex accounts of viewers, which clarify the ways in which many of them decode the visual texts they see online.

\section{References}

Bauer, J., \& Gradus, R. (Writers). (2015). Hot girl wanted. In J. Bauer (Producer). United States.

Bindel, J. (2010). The truth about the porn industry. Retrieved from https://www. theguardian.com/lifeandstyle/2010/jul/02/gail-dines-pornography 


\section{Conclusion}

Bridges, A. J., Wosnitzer, R., Scharrer, E., Sun, C., \& Liberman, R. (2010). Aggression and sexual behavior in best-selling pornography videos: A content analysis update. Violence Against Women, 16(10), 1065-1085.

Brooks, S. (2010). Hypersexualization and the dark body: Race and inequality among black and Latina women in the exotic dance industry. Sexuality Research and Social Policy, 7(2), 70-80.

Brownmiller, S. (1975). Against our will. New York, NY: Simon and Schuster.

Cowan, G., \& Campbell, R. (1994). Racism and sexism in interracial pornography. Psychology of Women Quarterly, 18, 323-228.

DeKeseredy, W. (2015). Critical criminological understandings of adult pornography and woman abuse: New progressive directions in research and theory. International Journal of Crime Justice and Social Democracy, 4(4), 4-21.

DeKeseredy, W., \& Hall-Sanchez, A. (2017). Adult pornography and violence against women in the heartland: Results from a rural Southeast Ohio study. Violence Against Women, 23(7), 830-849.

Dines, G. (2003). From fantasy to reality: Unmasking the pornography industry. In R. Morgan (Ed.), Sisterhood is forever: The women's anthology for a new millennium (pp. 306-314). New York, NY: Washington Square Press.

Dines, G. (2006). The white man's burden: Gonzo pornography and the construction of black masculinity. Tale Journal of Law and Feminism, 283, 283-297.

Dines, G. (2010). Pornland: How porn has hijacked our sexuality. Boston, MA: Beacon Press.

Dines, G. (2016). Is porn immoral? That doesn't matter: It's a public health crisis. The Washington Post, April 8, 2016, Retrieved on April 18, 2018.

Dines, G., Jensen, R., \& Russo, A. (1998). Pornography: The production and consumption of inequality. New York, NY: Routledge.

Dodige, N. (2007). The brain that changes itself: Stories of personal Triumph from the frontiers of brain science. London, UK: Penguin Books.

Dworkin, A. (1989). Pornography: Men possessing women. New York, NY: Dutton.

Dworkin, A., \& MacKinnon, C. (1988). Pornography and civil rights: A new day for women's equality. Minneapolis, MN: Organizing Against Pornography.

Fitzgerald, K., \& Grossman, K. (2017). Sociology of sexualities. New York, NY: Sage.

Foucault, M. (1992). The perverse implantation. In E. Stein (Ed.), Forms of desire: Sexual orientation and the social constructionist controversy (pp. 11-24). New York, NY: Routledge.

Fradd, M. (2017). The porn myth: Exposing the reality behind the fantasy of pornography. San Francisco, CA: Ignatius Press.

Hagedron, J. (1997). Asian women in film: No joy, no luck. In S. Biagi \& M. Kern-Foxworth (Eds.), Facing difference: Race, gender, and mass media (pp. 90-103). New York, NY: Routledge.

Hilton, D., \& Clark, W. (2011). Pornography addiction: A neuroscience perspective. Surgical Neurology International, 2, 19-23.

Ketcham, J. (2014). I am Jennie. New York, NY: Simon \& Schuster.

Loftus, D. (2002). Watching sex: How men really respond to pornography. New York, NY: Thunder's Mouth.

McKee, A. (2005). The objectification of women in mainstream pornographic videos in Australia. Journal of Sex Research, 42(4), 277-290.

McKee, A. (2015). Methodological issues in defining aggression for content analyses of sexually explicit material. Archives of Sexual Behavior, 44, 81-87. 
McKee, A. (2016). Pornography as a creative industry: Challenging the exceptionalist approach to pornography. Porn Studies, 3(2), 107-119.

Miller-Young, M. (2010). Putting hypersexuality to work: Black women and illicit eroticism in pornography. Sexualities, 13(2), 219-235.

Miller-Young, M. (2014). A taste of brown sugar. Durham, NC and London, UK: Duke University Press.

Monk-Turner, E., \& Purcell, C. (1999). Sexual violence in pornography: How prevalent is it? Gender Issues, 17, 58-67.

Nakamatsu, T. (2005). Faces of "Asian brides": Gender, race, and class in the representations of immigrant women in Japan. Women's Studies International Forum, 28(5), 405-417.

Nowrojee, S., \& Silliman, J. (1997). Asian women's health: Organizing a movement. In S. Shah (Ed.), Dragon ladies: Asian American feminists breath fire. Boston, MA: Sutherland Press.

Paul, P. (2005). Pornified: How pornography is damaging our lives, our relationships, and our families. New York, NY: Henry Holt.

Paul, P. (2010). From pornography to porno to porn: How porn became the norm. In J. Stonder \& D. Hughes (Eds.), The social costs of pornography: A collection of papers (pp. 3-20). Princeton, NJ: Witherspoon Institute.

Picker, M., \& Sun, C. (Writers). (2008). The price of pleasure: Pornography, sexuality \& relationships. Northampton, MA: Media Education Foundation.

Reece, R. (2015). The plight of the black Belle Knox: Race and webcam modelling. Porn Studies, 2, 269-271.

Russell, D. (1988). Pornography and rape: A causal model. Political Psychology, 9(1), 41-73.

Smith, J. (2013). Porn has changed - For the worse. Even men have noticed. Retrieved from http://www.independent.co.uk/voices/comment/porn-has-changed-forthe-worse-even-men-have-noticed-8846457.html

Sun, C., Wosnitzer, R., Bridges, A. J., Scharrer, E., \& Liberman, R. (2010). Harder and harder: The content of popular pornographic movies. In M. Antoinette Paludi \& F. Denmark (Eds.), Victims of sexual assault and abuse: Incidence and psychological dimensions (pp. 335-361). Westport, CT: Praeger.

Tarrant, S. (2016). The pornography industry: What everyone needs to know. Oxford, UK: Oxford University Press.

Uchida, A. (1998). The orientalization of Asian women in America. Women's Studies International Forum, 21(2), 161-174.

Voss, G. (2015). Stigma and the shaping of the pornography industry. London, UK: Routledge.

Wagoner, B. (Writer). (2012). After porn ends. In O. E. A. M. Media (Producer). United States: Gravitats Ventures (US).

Zhou, Y., \& Paul, B. (2016). Lotus blossom or dragon lady: A content analysis of "Asian women" online pornography. Sexuality \& Culture, 20, 1083-1100. doi:10.1007/s12119-016-0375-9. 

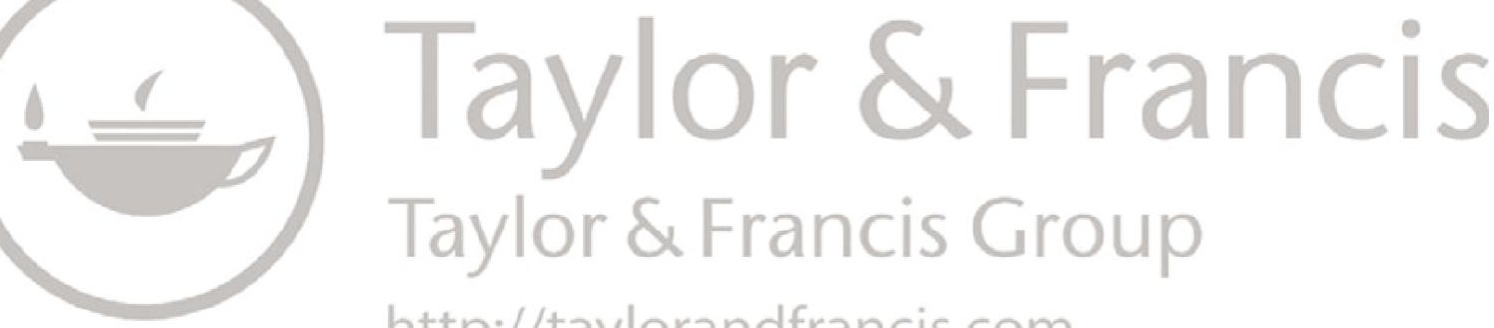

http://taylorandfrancis.com 


\section{Index}

Note: Bold page numbers refer to tables; Italic page numbers refer to figures and page numbers followed by 'n' refer to end notes.

the Abilene Paradox 85

adolescents: defined 20n2; pornography's effects on $48-50$

adult film industry: and Internet 11-12; use of DVDs 11; use of VHS 11; see also pornographic industry aggression: acts viewers perceiving as 67-69; coding, in same-sex videos 32-33; defined 31; displays in pornography, coding 30-32; in female/ female videos 100-101; majority of mainstream pornography containing 64-69; in male/male videos 97-99; men's preferences regarding 83-87; and number of views 79-80; pornography viewers' reflections about 114-117; prevalence in PornHub videos 65; role in pornography 2 ; scholarly claims about popularity of 77-78; and video rating 80-81; women watching pornography seeking $88-89$

Alexa Internet 27

amateur pornography 15-16

anti-pornography literature 77

anti-pornography scholars: on pornography 3

Archives of Sexual Behavior 5

Asian women 110; literature on, in media 110,119 ; literature on, in porn 110 ; non-consensual violence and 111, 111-112; as passive doll-like "fucking machines" 119, 131; pornographic depictions of 110

"authentic" consent 31

Bader, Michael 48

Barely Legal magazine 17 the $B B C 64,70$

behaviors, and pornography viewers on fantasies 123

bell hooks 109

Blue Movie 10

bondage, discipline, dominance, and submission (BDSM) 5, 20; mainstreaming of 18-19

Boyle, Karen 70

Bridges, A. J. 31, 124-125

Brooks, D. 108

de Cadenet, A. 14

Campbell, R. 108-109

catfight 102

childification of women 17

Collins, Patricia Hill 108-109

Connelly, Tim 11

consent: authentic 31; performed 31

consumers: feminists on effects of pornography 42-44; pornography effects on $42-54$

Cowan, G. 108-109

Craigslist 36

cultural "pornification" 12

The Daily Telegraph 64, 70

Damiano, Gerard 10

Deep Throat 10

"democratization" of online pornography $15-16$

deviant sexuality 71

"digital natives" 18

Dines, Gail 16, 17, 64, 70, 78, 108, 110, 125,132

Doidge, Norman 70, 78

dopamine 78 


\section{Index}

ethnicity: of performers 115;

pornographic sexual aggression, role in 108-109; pornography viewers' reflections about 114-117; see also race/ethnicity

Facebook 36

fantasies: hetero-male 102; sexual 132; viewers on behaviors and 123

female/female (f/f) pornography: aggression in 100-101; non-consensual aggression in 130; previous research on aggression in 94; visible aggression in 130

feminists: debates over pornography and its effects 42-44; liberal 44; radical 4 ; and rape culture 43; sex-positive 43-44; see also women

feminists sex wars 42,92

Fifty Shades of Grey (James) 18-19

Flynt, Larry 17

"forced sex" 18; see also aggression; sexual aggression

Gaddam, S. 17

Gagnon, J. H. 44

gay masculinity 103

gender inequality: and pornography

45-50; and pornography-aggression relationship 48

"golden age" of porn 10

"gonzo" pornography 16

Gossett, J. L. 119

Grey, Sasha 12, 17

The Guardian 64, 70, 132

Hald, G. M. 48

Hedges, Chris 70

heterosexual pornography: aggression, common in 94-96; power dynamics in 92; see also pornography

Hot Girls Wanted 16

The Independent 64, 70, 129

Internet: and adult film industry 11-12; free pornography on 12 ; pornography $64,77-78$

James, E.L. 18

Jameson, Jenna 12

Japanese porn industry 113,118

Jensen, Robert 13, 43, 64, 70

Journal of Sex Research 5
Kalish, R. 102

Kardashian, Kim 12

Kijiji 36

Kimmel, M. 102

Kipnis, Laura 43

Latina women 110; media depictions of 110; non-consensual aggression by racial combination 113; partnered with 113, 113; portrayals of affection toward 111, 111; visible aggression and 111

lesbianism 102

Levy, D. 17

liberal feminists 44

Löfgren-Mårtenson, L. 18, 54

Loftus, D. 126-127, 129

mainstreaming: of BDSM 18-19; pornography $12-13$ see also mainstream pornography

mainstream online pornography: getting "harder and harder" 69-73; porn becoming more aggressive $72-73$; viewers' perceptions about "harder and harder" argument 71-72

mainstream pornography: acts perceived as aggression 67-69; becoming aggressive 125-126; prevalent aggressive acts 66-67; viewers and preference on aggressive content 78-81

Makin, D. A. 13, 17, 19

Malamuth, N. M. 48

male/male $(\mathrm{m} / \mathrm{m})$ pornography: aggression in 97-99; previous research on aggression in 93-94

Månson, S. A. 18, 54

"masculinization of sex" 102

McKee, A. 31, 32, 50, 80, 129-130

McKeown, J. K. 15

men, and preferences regarding aggression 83-87

Miller-Young, M. 110

MindGeek 12, 28

Monk-Turner, E. 108, 109

Morczek, A. L. 13, 17, 19

Morgan, Robin 43

Mulholland 50

myths, on popularity of aggression: scholarly claims about popularity of aggression 77-78; viewers and their preferences 82-89; viewers preferring aggressive content $78-81$ 
myths, on prevalence of aggression: mainstream online pornography getting "harder and harder" 69-73; mainstream pornography containing aggression 64-69

\section{New York Times 70 \\ non-consensual aggression 65-66, 68, 72-74, 80-81, 84-85, 101, 103, $112-113,122-127,130$ \\ normalization of pornography 13 \\ North American industry 113 \\ Ogas, O. 17 \\ online pornographic videos: coding procedures and inter-coder reliability 34-35; content analysis 27-35; measurement and coding 30-34; sampling strategy and procedure 27-30; see also Internet \\ The Other Hollywood 11}

Parrott, S. 118

Paul, B. 108, 110-111, 119, 131

Paul, Pamela 70, 78

"performed" consent 31

Peter, J. 18, 49

Playboy 17

pleasure: role in pornography 2

PornHub 5, 11-12, 14, 27-30, 65; prevalence of aggression in videos of 65; prevalence of specific aggressive/ degrading acts in 67; sampling videos within 28-30, 29

"porn literacy" 51, 123

"porno chic" 10, 15

pornographic industry: historical shifts in 10-12; technological developments in 10-12; see also adult film industry pornography: defined 10; effects on consumers $42-54$; and feminists 42-44; and gender inequality 45-50; mainstreaming 12-13; normalization of 13; potential beneficial effects of 50 ; self-perceived effects of 51-54; and sexual aggression 45-50; sexual script theory 44-45; and sexual violence 45-50; as sociological phenomenon 1; stance on 4-5; use and contents 13-19; use and effects 1-2; writings on 2 pornography scholars: and antipornography organizations 3 ; on pornography 3 pornography users: interviewees' sample 37-38; interviews with 35-39; procedure of interviews 38-39; sampling strategy and recruitment 35-38

pornography viewers: on fantasies and behaviors 123; reflections about race, ethnicity, and aggression 114-117 "pornostyle" fashion 13

Porn Studies 4

The Price of Pleasure 69

"pro-am" pornography 16

Purcell, C. 108, 109

race/ethnicity: and pornographic sampling 30, 33-34; pornography viewers' reflections about 114-117

race myths: overview 108-109; racial minority women and aggression 111-114; theory and research on visible minority women in media 109-111; theory and research on visible minority women in pornography 109-111; viewers' reflections about aggression 114-117; viewers' reflections about ethnicity 114-117; viewers' reflections about race 114-117

racialized sexual exploitations 117

racial minority women: popular online pornography, content analysis 111-114; suffering highest rates of aggression 111-114

"radical feminists" 4

Ramirez, J. M. 31

RedTube 12, 28

Reece, R. 110

research: on aggression in (f/f) pornography 94 ; on aggression in $(\mathrm{m} / \mathrm{m})$ pornography $93-94$; on visible minority women in media and pornography 109-111

Ruberg, B. 15

Rubin, Gayle 43

same-sex pornography: aggression in female/female videos 100-101; aggression in male/male videos 97-99; viewers on 96-101

same-sex videos 103n2; coding aggression in 32-33; more aggression than heterosexual videos 130; sampling for 30 
self-perceived effects of pornography $51-54$

"sex-positive feminism" 43-44

sex-positive feminists $43-44$

sexual aggression: correlational studies 46-47; experimental studies 45-46; and pornography 45-50; qualifying and moderating factors in pornography and 48-49; see also aggression

sexually explicit materials (SEM) 31 sexual orientation myths: aggression in female/female (f/f) pornography 94; aggression in male/male $(\mathrm{m} / \mathrm{m})$ pornography 93-94; heterosexual pornography, aggression most common in 94-96; overview 92-93; viewers on same-sex pornography and aggression 96-101

sexual script theory 44-45

sexual violence: correlational studies 46-47; experimental studies 45-46; and pornography 45-50; see also sexual aggression

sex wars 3,42

Shulevitz, Judith 70

Simon, W. 44

Smith, Joan 129

spanking 68, 73-74, 80, 83-88, 95, 95, 123-124

The Sydney Morning Herald 64, 70

Tajima, R. 110

theory: on visible minority women in media 109-111; on visible minority women in pornography 109-111

Titcomb Parrott, C. 118

Valkenburg, P. M. 18, 49

verbal aggression 79, 79-80, 94-95, 101-102, 130

videocassettes, and pornography 10-11

Video Home System (VHS) 11

video rating, and aggression 80-81 videos: containing aggression 124-125; featuring women of color and aggression 130-131

viewers: on consent and mutual pleasure 123-124; men's preferences regarding aggression 83-87; perceptions on "harder and harder" argument 71-72; prefer to watch less aggression 126-127; prefer videos female performers expressing pleasure 128-130; on samesex pornography and aggression 96-101; and their preferences 82-89; women on pornography and aggression $88-89$

Village Voice 43

violence: non-consensual and Asian women 111, 111-112; sexual 45-50

violent behavior see sexual aggression

visible aggression 66, 113

"Vivid girls" 15

Walker, A. 13, 17

Warhol, Andy 10

The Washington Post 64, 70

Web 2.0 12, 20n1

WGCZ Holding 12

Whisnant, R. 78

Williams, Linda 4

Willis, Ellen 43

women: childification of 17 ; more likely to express interest in aggression 127-128; and pornography content 14; viewership of pornography 14-15; watching pornography and seeking aggression 88-89; see also feminists

young consumers, and pornography 16-18

young performers, and pornography 16-18

YouPorn 12, 28

youth: pornography's effects on $48-50$; see also adolescents

Zhou,Y. 108, 110, 111, 119, 131 